\title{
Synthesis of Progressive Varying Texture Through Blending Laplacian Pyramid Coefficients
}

\author{
by

\section{Das Moitry} \\ A thesis submitted to the \\ Faculty of Graduate and Postdoctoral Affairs \\ in partial fulfillment of the requirements for the degree of
}

Master of Computer Science

Ottawa-Carleton Institute for Computer Science

The School of Computer Science

Carleton University

Ottawa, Ontario

September, 2020

(C) Copyright

Das Moitry, 2020 
The undersigned hereby recommends to the

Faculty of Graduate and Postdoctoral Affairs acceptance of the thesis

\title{
Synthesis of Progressive Varying Texture Through Blending Laplacian Pyramid Coefficients
}

\author{
submitted by Das Moitry \\ in partial fulfillment of the requirements for the degree of \\ Master of Computer Science
}

Professor David Mould, Thesis Supervisor

$\overline{\text { Professor Oliver van Kaick, School of Computer Science }}$

Professor Jochen Lang,

School of Electrical Engineering and Computer Science

Professor Anil Somayaji, Chair, School of Computer Science

Ottawa-Carleton Institute for Computer Science

The School of Computer Science

Carleton University

September, 2020 


\section{Abstract}

We generate a texture synthesis algorithm where there is a gradual progression of merged textures in the output image. The goal of the algorithm is to merge the textures based on the given measure of texture proportion. The proportion of texture contents defines how much of the textures from each input will be preserved in the output and how the distribution of texture will occur after merging. By merging two textures, a continuous and seamless output texture is formed. We design the algorithm in such a way, that both the local high and low frequency texture contents are preserved in the output.

We generate the Laplacian pyramids for each input. At each level of the pyramids we blend the coefficients from the inputs using the weighted smooth maximum function. The algorithm is applicable for any random phase textures. It is also applicable for some non-random phase textures which are stochastic and there are no well-defined visual structures.

Our method of progressively variant texture synthesis is distinguished from previous techniques by being able to produce a gradual as well as a scattered distribution of the merged textures in the output. This irregular distribution gives a realistic view in the merged region of the output. The transition of textures is not sudden rather it is smooth, coherent and continuous. 


\section{Acknowledgments}

I want to thank all the members of the Graphics, Imaging and Games Lab (GIGL) for welcoming me and giving me a very friendly working environment in the university. The lab meetings that happen at the GIGL are very informative and I found those really inspiring. Throughout my whole thesis journey I got a lot of advise, assistance from the lab members.

I want to convey my utmost gratitude to the most important person actively contributing towards the successful completion of my Masters degree - my supervisor: Prof. David Mould. He has guided me through all the necessary steps that I had to take to accomplish my thesis. He actively took interest in teaching me how to be thorough, systematic and meticulous in both my experiment and writing. He has been patient and considerate towards all my problems, mistakes and shortcomings throughout my thesis work and helped me at his best to overcome those. It has been a great learning experience for me while working with Prof. David Mould. 


\section{Table of Contents}

Abstract $\quad$ iii

Acknowledgments $\quad$ iv

Table of Contents $\quad$ v

List of Tables viii

List of Figures $\quad$ ix

1 Introduction 1

1.1 Goal ........................... 2

1.2 Thesis Overview . . . . . . . . . . . . . . . . 2

1.3 Statement of Contributions . . . . . . . . . . . . 3

2 Previous Work 5

2.1 Procedural Techniques . . . . . . . . . . . . . . . 5

2.2 Example Based Algorithms . . . . . . . . . . . . . . . . 6

2.2.1 Pixel Based Algorithms . . . . . . . . . . . . . 6

2.2.2 Patch Based Algorithms . . . . . . . . . . . . . . . . . . 7

2.2.3 Optimization Based Algorithm . . . . . . . . . . . 8

2.3 Statistics-based Texture Synthesis . . . . . . . . . . . . . . 8

2.4 Algorithm Using Image Pyramid and Noise Image . . . . . . . . . . . 9

2.5 Algorithms Using Convolutional Neural Network . . . . . . . . . . . . 9

2.6 Algorithms Based On User Inputs . . . . . . . . . . . . . . . . . . . . 10

2.7 Progressively Variant Texture Synthesis Methods . . . . . . . . . . . 11

2.8 Relevance Of Previous Methods To Our Algorithm . . . . . . . . . . 12 
$\begin{array}{llr}3 & \text { Background } & 15\end{array}$

3.1 Random Phase Texture . . . . . . . . . . . . . . . . . 15

3.2 Histogram-Preserving Blending Operator . . . . . . . . . . . . . 16

3.2.1 Tiling Of Input Image And Blending Of Patches From Input . 19

3.2.2 Inverse Transformation . . . . . . . . . . . . . . . 21

3.2.3 Results Of Histogram-Preserving Algorithm . . . . . . . . . . 22

3.3 Laplacian Pyramid . . . . . . . . . . . . . . . . . . . . . . 24

3.4 Smooth Maximum Function . . . . . . . . . . . . . . . . . 24

3.5 SLIC Superpixel . . . . . . . . . . . . . . . . . . . . 25

4 Random Phase Texture Transition With Smooth Maximum 27

4.1 Pipeline Of The Steps Of Our Algorithm . . . . . . . . . . . . . 27

4.2 Algorithm . . . . . . . . . . . . . . . . . . . . . . 31

4.2.1 Blending Coefficients . . . . . . . . . . . . . . . . . 31

4.2 .2 Joint SLIC Segmentation . . . . . . . . . . . . . . . . . 37

4.2.3 Segmentation With The SLIC Superpixel . . . . . . . . . . . . 40

4.2.4 Assigning Color And Smooth Merging At Halo . . . . . . . . . 44

5 Results and Discussion $\quad 49$

5.1 Outcome With Different Textures And Evaluation . . . . . . . . . . 49

5.1.1 Results With Different Types Of Inputs . . . . . . . . . . . . 50

5.1.2 Results without using the SLIC segmentation . . . . . . . . . 58

5.1.3 Results With Progressively Variant Texture Contents . . . . . 59

5.1.4 Results Of Blending Two Textures With Different Textureness 61

5.1.5 Results Showing The Effect Of Sizes Of The SLIC Segments . 62

5.1.6 Results With Two Dissimilar Inputs in Terms of Colors and Texture Contents . . . . . . . . . . . . . . 66

5.1.7 Results With Two Similar Inputs In Terms of Colors and Texture Contents . . . . . . . . . . . . . . . . 67

5.1.8 Texture Distribution Map . . . . . . . . . . . . . . . 71

5.2 Discussion . . . . . . . . . . . . . . . . . . . 72

5.3 Timing Of Different Steps of Algorithm . . . . . . . . . . . . . 73

5.4 Failure Cases . . . . . . . . . . . . . . . . . . . . . . 74 
$\begin{array}{llr}6 & \text { Conclusion } & 77\end{array}$

6.1 Summary . . . . . . . . . . . . . . . . . . . . 77

6.2 Future Work . . . . . . . . . . . . . . . . . . . . . 78

$\begin{array}{lr}\text { List of References } & 80\end{array}$ 


\section{List of Tables}

4.1 Symbol Definitions . . . . . . . . . . . . . . . . . 33

5.1 Timing of algorithm $\ldots \ldots \ldots \ldots \ldots \ldots \ldots \ldots$ 


\section{List of Figures}

2.1 The result of synthesis of Progressively-Variant Textures on Arbitrary Surfaces [42] (top) and result from Wei's method [40](bottom). . . . . 12

2.2 The result of texture synthesis by Heeger and Bergen. The process synthesizes by histogram matching of the input, applying on uniform white noise and using image pyramid [21]. . . . . . . . . . . . . . . . 13

3.1 By mapping the random RGB values(upper right image) with the input image(upper left image) using the OT solver, the image with the same structure as the input is obtained (the bottom image) . . . . . . .

3.2 Hexagonal patches are chosen from any random locations in the gaussianized input(left). In the output plane (right), inside each triangle formed by intersecting 3 hexagons, 3 patches from the input are blended $[22] . \ldots \ldots \ldots \ldots \ldots$

3.3 In the blending equation, the barycentric co-ordinates $w_{A}, w_{B}$ and $w_{C}$ are determined for vertices A, B, C respectively . . . . . . . . . . . . 21

3.4 Results of Histogram preserving blending algorithm . . . . . . . . . 23

4.1 The generated output of our algorithm which demonstrates a gradual transition of textures from the left image to textures from the right image . . . . . . . . . . . . . . . . . .

4.2 Sample inputs for representing the outcomes for different values of $p, q$ and $t$ in smooth maximum function. . . . . . . . . . . . . . 35

4.3 Greyscale output keeping the value of $t$ constant throughout the whole output image. . . . . . . . . . . . . . . . . .

4.4 Greyscale output combining the levels of resultant pyramid and changing $t$ from 0 to 1 from the left edge to the right edge of output image plane. 
4.5 Output after the smooth maximum function and reconstruction from Laplacian pyramid where individual texture contents from inputs are not recognizable in the greyscale output. . . . . . . . . . . . . . .

4.6 Images at the upper row are two inputs. Bottom left is the colored result without using the SLIC segmentation. Bottom right is the colored result after using the SLIC segmentation. . . . . . . . . . . . . .

4.7 Sample image explaining how the SLIC segmentation works in our experiment. SLIC segmentation is done in any one of the inputs but in the segmentation formula, color distances of pixels at same location in both inputs, from their corresponding centroid location are considered. Color distances are measured with Eqn. 4.8 and Eqn. 4.9. . . . . .

4.8 The SLIC segments map. Here the regions with grey marking are populated with right image contents and white regions are populated with left image contents. Contents from from both inputs are evenly distributed except at the left most and right most corner. . . . . .

4.9 SLIC segments boundary edges are noticed subtly (shown inside circles) if two texture elements across the boundary are very much dissimilar in color and contents . . . . . . . . . . . . . . . .

4.10 Some SLIC segments are very subtly visible (in Fig. (c))if two different colors are present on each side of the SLIC segments. . . . . . . . . .

4.11 Boundary edges are subtly visible before the alpha blending (left image). After the alpha blending of colors along the edges of the SLIC segments, the boundary edges of segments are not visible (right image). 47

4.12 This is a zoomed representation of a SLIC boundary. Along the boundary pixels, $\left(x_{i-n}, y_{i-n}\right), . .,\left(x_{i}, y_{i}\right), . .\left(x_{i+n}, y_{i+n}\right)$, alpha blending of colors

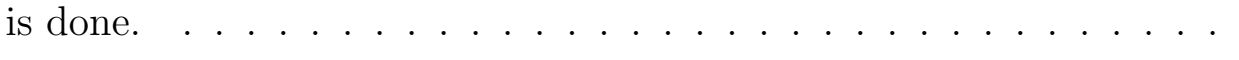

5.1 Results at the middle column showing the blended image of two inputs at left and right. The inputs are generated by the histogram preserving

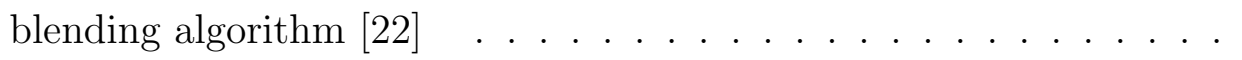

5.2 Results at middle column showing blending of non-random phase textures(left and right column are inputs [25], [24], [41], [15]). The inputs are used directly without using histogram preserving algorithm . . . . 
5.3 Results at middle column showing blending of non-random phase textures(left and right column are inputs). The inputs are used directly without using histogram preserving algorithm . . . . . . . . . . . . 54

5.4 Results at middle column showing blending of non-random phase textures(left and right column are inputs). The inputs are used directly without using the histogram preserving algorithm . . . . . . . . . .

5.5 Results of our algorithm at the middle column with both direct input and input generated by histogram preserving algorithm(inputs are at left and right column) . . . . . . . . . . . . . .

5.6 Results at middle column showing blending of arbitrary inputs(left and right column are inputs). The inputs [37], [6], [36], [20] are used directly without using the histogram preserving algorithm . . . . . . . . .

5.7 Results at the middle column are generated without using the SLIC segmentation. The inputs at the left and the right column are generated using the histogram-preserving algorithm. . . . . . . . . . . .

5.8 Results at middle column showing progressively variant textures from left to right edge of output image plane. . . . . . . . . . . . . . . . . 60

5.9 Result of blending two textures with different textureness. The images in the upper row are inputs. The bottom image is the output. . . . .

5.10 Result of blending two textures with different textureness. The images in the upper row are inputs. The bottom image is the output. . . . .

5.11 Results of our algorithm showing the effect of the different sizes of the SLIC segments. There is change of shape and size of the texture patches according to the size of the SLIC segments. The images in the uppermost row are inputs. The images in the second and third rows are the outputs. . . . . . . . . . . . . . . . .

5.12 Output showing effect of different sizes of SLIC segments. The images in the upper row are inputs. The images in the second and rows are the outputs. Sizes of the SLIC segments are comparatively small in the left output compared to the sizes in the right output. . . . . . . .

5.13 Result of our algorithm at the bottom row. Inputs at the first row are generated after the histogram preserving blending operation . . . .

5.14 Result of our algorithm at the bottom row. Inputs at the first row are generated after the histogram preserving blending operation . . . . 
5.15 Result at the bottom row showing the blend of two similar inputs at the upper row. The inputs are similar in terms of color and texture

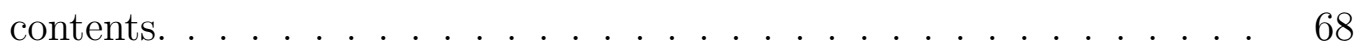

5.16 Original Inputs $\ldots \ldots \ldots$

5.17 Results of the histogram-preserving blending which we used as inputs for our algorithm. . . . . . . . . . . . . . . . . 69

5.18 Sharp edges observed before smooth blending at halo region (the left image). Result after smooth merging along the SLIC boundaries (the

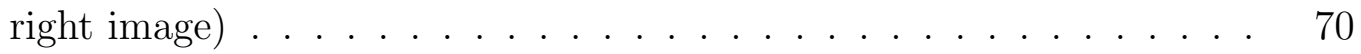

5.19 Result(at the bottom left) after texture blending where the texture distribution from inputs are based on the user defined texture distribution map (at the bottom right). . . . . . . . . . . . . 72

5.20 Result of blending two textures with different textureness . . . . . . 75

5.21 Result of blending two textures with regular structure . . . . . . 76 


\section{Chapter 1}

\section{Introduction}

Texture is an important spatial feature, useful for identifying objects or regions of interest in an image [39]. Texture is usually defined as an infinite repeating pattern that may vary locally but the statistical properties don't vary based on the location. In computer graphics and image processing algorithms, texture has been defined in many ways. Some definitions are based on particular applications, some are perceptually motivated and some are driven completely by the application in which the definition will be used.

Texture synthesis is used for image compression applications, rendering textures on object surfaces in computer graphics and many other purposes. In the process of texture synthesis, texture blending and merging are sometimes the intrinsic steps. In this thesis we have approached one such process of texture synthesis for structureless stochastic texture. Stochastic texture is a type of texture where there are no long correlated and recognizable features. There are no regular patterns or well defined structures in stochastic textures. Examples of such natural textures are sand, moss, granite, lava textures.

The main application of texture synthesis is derived from the texture mapping on a computer generated graphic or 3D model. Sources of texture images are usually scanned photographs and available photographs. But these textures in photographs are too small to cover the entire object surface. Therefore, texture is synthesized according to the required dimension. A simple tiling will give rise to different types of unacceptable artifacts. Some of the basic artifacts include visible repetitions and seams. Texture synthesis can solve these basic problems regarding the size, 
repetitions and seams. Texture synthesis is also applied in other image processing fields such as image or video compression, occlusion filling in stereo vision.

The process of texture synthesis sometimes involves the merging or blending of two or more different types of textures in a single image. In those cases the blending of textures should be smooth, natural and visually unobtrusive. Our work focuses on the merging of the two input textures. The goal is to create a smooth gradual texture transition from the textures of one input to that of the other input while merging.

\subsection{Goal}

The goal of our texture synthesis algorithm is to generate non-homogeneous stochastic textures by merging two input textures. We want to design our algorithm in such a way that, the proportion of the two input textures can vary spatially in the merged output and this variation will be user controlled. The distribution of textures can follow any distribution map specified by the users. The distribution map will indicate the desired proportion of the input textures and how the textures will be distributed in the output. In this thesis, we will mostly generate outputs where there is a linear progression of textures contents in the output. After merging, there will be a smooth, gradual linear progression of textures from the left edge of the output towards the right edge. There will be smooth transition of textures and the merged texture will be continuous; without any sudden change of texture contents from one input to that of the other input. It should be seamless and visibly unobtrusive.

\subsection{Thesis Overview}

Our method first takes small inputs of random phase textures. For generating a large output of any required size, we use the algorithm of high performance by-example noise using a histogram preserving blending operator generated by Eric Heitz and Fabrice Neyret [22]. We also experiment with some stochastic textures as input directly, without using the histogram preserving blending algorithm.

Then we generate a segmentation map using the Simple Linear Iterative Clustering (SLIC) algorithm. For each segment in the segmentation map, we generate 
two Laplacian pyramids for the two inputs. Then at each level of the Laplacian pyramids we blend the coefficients using the weighted smooth maximum function. We compute the weights based on two factors. One factor is the measure of the proportion of texture contents in the output from each input. The other factor is the textureness in each input. Textureness denotes the local high frequency content in the image [4]. We compute the weights in such a way that the textures with low textureness are not lost or replaced in the output. Based on the weights, the output of the smooth maximum function is obtained.

For each of the SLIC segment, we get a resultant pyramid with the blended coefficients. Combining the levels of the resultant pyramid we get a greyscale blended segment. If there are $K$ number of segments in each input then we get $K$ blended segments. All of the blended segments together constitute the output greyscale image. For assigning colors to the greyscale output, we use a precomputed lookup table of each input. The lookup table is generated in the histogram preserving blending algorithm [22].

\subsection{Statement of Contributions}

We present a framework for merging stochastic textures with local control over the degree to which one texture or the other is more prominent. The outputs have features from both inputs, with strong features being preserved in a perceptually appealing way. Supporting this work, this paper makes the following technical contributions:

- Structural texture blending through use of the smooth maximum to mix coefficients of the Laplacian pyramid, suitable for stochastic textures.

- Adding texture heterogeneity through a joint SLIC segmentation of both input textures.

- Use of a weighted smooth maximum for controllable and spatially varying texture mixing.

- Overall framework for synthesizing non-homogeneous stochastic textures from exemplars. We use histogram-preserving blending to generate full-size textures 
from exemplars, then blend the two textures in a Laplacian pyramid representation. 


\section{Chapter 2}

\section{Previous Work}

In this section we are going to write about some of the notable texture synthesis algorithms. We also present some work related to the progressively variant texture synthesis and how these processes are different from ours.

\subsection{Procedural Techniques}

Throughout the history of computer graphics, various methods of texture synthesis are being used. From the early beginnings, the procedural techniques are being used for powerful modelling and synthesis of texture. Procedural techniques specify some characteristics of a computer-generated model or effect. In this approach, rather than explicitly specifying and storing all the complex details of a real texture, it abstracts them into an algorithm or function. This helps in creating inherent multi-resolution textures that can be evaluated to the resolution needed. It also provides the parametric control and flexibility [10]. Perlin Noise is the function formulated by Ken Perlin and it is one of the building blocks for procedural texture synthesis. It is the basis for an interactive synthesizer for designing highly realistic computer generated texture. Perlin introduced the concept of "solid texture" in Computer Graphics Imagery(CGI). This is used to create very convincing representation of clouds, fire, water, stars, marble, wood, rock etc [34].

Another example fo procedural technique is the Local Random Phase(LRP) Noise. It addresses the problem of preserving the structural features by storing the frequencies and phase information of the features [19]. The LRP model is the blending of local noises centered on a regular spatial lattice. The local noises are 
sums of cosines with random phase. The LRP noise model is extended by using locally defined and controlled spot noise [33]. The spot noise model improves the control over local structural features. This model focuses on structures which can be defined by a repetitive structured kernel function. The kernels are constructed with the base component of the features.

There is a procedural method for texture synthesis which is biologically motivated, called reaction-diffusion method [38]. This method produces textures which directly matches the geometry of a surface like clusters of spots on leopards, web-like design on giraffes. The method expanded the range of textures that is produced by a cascade of the number of reaction-diffusion procedure. The procedure lays down an initial pattern and then the subsequent steps refine the pattern.

\subsection{Example Based Algorithms}

Exemplar-based texture synthesis aims at creating new texture images from an input sample that are visually similar to the input, but are not plain copy of it [13]. Pixelbased, patch-based and optimization based algorithms are some of the example-based algorithms. In this section we are mentioning some of those algorithms.

\subsubsection{Pixel Based Algorithms}

In the pixel-based algorithm, a new texture is synthesized pixel by pixel. Here value of each new pixel is derived from the values of its neighbouring pixels. This method is quite slow due to its extensive search of input neighbourhoods. Yet this method works on wide range of textures preserving some local image structures. [13]

Ashikhmin proposed a texture synthesis process which is suitable for some specific naturally occurring textures [3]. These are partly repeating patterns consisting of familiar objects having irregular size, such as bunch of small flowers clustered irregularly, bushes, small pebbles. In this algorithm, the synthesized image has the basic spatial frequencies of the input image and also visually similar to the inputs. The algorithm lets the user provide a target image using a painting-style interface which only traces some general properties of the input texture and the rest 
is achieved by the synthesis algorithm.

\subsubsection{Patch Based Algorithms}

In patch-based algorithm a patch is copied instead of copying pixels. Generally, in statistics-based methods, textures are characterized by a statistical model. However, in patch re-arrangement methods, for creating a new texture, heuristics are used to re-arrange parts of the sample texture in a random way. Efros and William generated a patch-based algorithm called image quilting [14]. In this process, the authors used dynamic programming to search for an optimal path to cut through the overlapped areas. The unit of synthesis is a square block, the size of which is specified by the user. The algorithm searches for a set of blocks satisfying the overlapping constraints within some error tolerance for every output location. The algorithm then randomly picks one of the sets of blocks. At the overlapped area between the chosen block and the former blocks, the error surface is measured. This is measured in order to make the cut through the overlapping blocks on the pixels where the texture matches the most. In this way through dynamic programming, the algorithm generates the minimum cost path. It makes the boundary of the new patch. It places the patch on the output and the process is repeated. This algorithm works very well on semi-structured texture which are generally hard to synthesize. The algorithm does not give desired output when there is less variability in the input texture. In that cases the output contains mismatched and deformed boundaries and there are many repetitions in the result [14].

Graph-cut technique was introduced to handle the overlapped regions of patches with dynamic programming [30]. To place the patch, the algorithm finds an appropriate location. Then graph cut method is used to find the optimal part of the patch to place on the output. The algorithm solves the problem as a minimum cost graph cut problem [30]. In tile based texture synthesis, the tiles are used as the unit of texture synthesis instead of the arbitrary shaped patches. There is no overlap between the tiles. The tiles are placed in the output in such a way that the texture patterns in the output are continuous across the edges of the tile [8]. 


\subsubsection{Optimization Based Algorithm}

The optimization-based algorithm is the combination of pixel-based and patch-based synthesis. The unit of synthesis is a single pixel. Here all the pixels are considered together and their value is determined by optimizing a quadratic error energy function. The optimization of texture quality is done with respect to a similarity metric. The similarity metric is defined locally. Then a global metric is formed by merging these local similarity measures. The global metric is used to optimize the whole texture globally. This is slower than previous algorithms because of iterative optimization. [29].

\subsection{Statistics-based Texture Synthesis}

Statistics-based texture synthesis methods proposed by Julesz are performed in two steps. The first step is - from the sample texture, a set of statistics is estimated. The second step is - subject to these statistical constraints, a random image is generated. This method motivated the generation of several other methods. These methods differ in the set of statistics considered and in the optimization methods which are imposed on random image. One such method is Random Phase texture synthesis, where a texture is generated by randomizing the Fourier phase while maintaining the Fourier modulus [17]. This algorithm is explained in details in our next chapter. Using this algorithm we have generated some results. We used some the results as the inputs of our algorithm. The Random Phase Noise method works for the textures having no salient details. These are called micro-texture. It fails for the textures with more structured contents [17].

Textures may share the same second and third order statistics while being visibly different [28]. Based on this, Julesz proposed a second theory to explain texture pre-attentive discrimination by introducing the textons [26], [27]. Textons refer to fundamental micro-structures in natural images and videos. According to Julesz second theory, only the first order statistics are relevant for texton perception. Images with same texton densities could not be discriminated. 


\subsection{Algorithm Using Image Pyramid and Noise Image}

Heeger and Bergen synthesize a solid texture that matches the appearance of the input texture sample [21]. The method analyses a digitized image and computes a the number of texture parameter values. Those parameter values are then used to synthesize a new image [21]. It synthesizes the textures by matching histograms of filter outputs. The method starts with a digitized texture image and a uniform white noise. Then it enforces the same histogram on the white noise image. It uses an invertible image representation called image pyramid. This method measures the marginal statistics and does not consider important correlations between pixels across scales and orientations. These correlations are important for generating edges and distinct patterns. Portilla and Simoncelli also used a noise image as input like Heeger and Bergen [21]. Portilla and Simoncelli proposed a universal statistical model for textures with respect to an complex wavelet transform [35]. The algorithm measures correlation and the expected product of their magnitude and the expected product of the fine scale coefficient with the phase-doubled coarse scale coefficient. The algorithm also include some marginal statistics of the image pixels and lowpass coefficients at different scales. Then it iteratively projects the image under construction onto the subspace of constraints using a gradient projection approach. The projection continues until the final stable output is obtained [35].

\subsection{Algorithms Using Convolutional Neural Net- work}

Convolutional Neural Network (CNN) has been used in some methods of texture synthesis. For some visual classification tasks, a completely trained CNN can be taken. By restricting the CNN to lower layers, a set of low level filters can be obtained which are used for the synthesis. By using CNN, the whole process of pre-determining the constraints and filters is not required. CNN directly encodes the filters [2]. For texture generation, a pre-trained CNN which worked on some related tasks can be directly used. The factors that affect the generated texture are - the selection of CNN, whether it is already trained, whether the parameters or 
weights are random or not. Through backpropagation of the CNN architecture, the statistics of all filters can be controlled at the same time. Leon Gatys used CNN in their method for texture generation [18]. This method is generalization of the Julesz model. It suggests that the correlation between neural network features describe the textures. For a given input texture one should generate the new sample of the texture. This is equivalent to starting with random noise and impose the correlation between the features.

\subsection{Algorithms Based On User Inputs}

There are some globally variant textures which are different from local and stationary texture. These are some natural textures which do not follow the stationary texture properties. These textures get affected by some external variables. For example, in texture of rusty iron, cracked paint, decay metal background where texture patterns depend on thickness of paint stroke, rust distribution. These textures have some local properties but no stationary properties. For these types of textures there are some texture synthesis processes based on the user input.

There is a texture synthesis algorithm called Image Analogies, which is controlled by the user input [23]. An image pair, consisting of an unfiltered image $\mathrm{A}$ and a filtered image $A^{\prime}$ is given. Another unfiltered target image $\mathrm{B}$ is also given as input. The algorithm generates an analogous filtered output texture $B^{\prime} . B^{\prime}$ relates to $\mathrm{B}$ in the similar way as $A^{\prime}$ relates to A. The algorithm initially constructs the Gaussian Pyramid of $\mathrm{A}, A^{\prime}$ and B. Then Gaussian pyramid of $B^{\prime}$ is computed, one level at a time. At each level, the statistics about each pixel $q$ in the target pair is compared to statistics about each pixel $\mathrm{p}$ in the input pair. For the maximum matching pixel $\mathrm{p}$, the feature vector corresponding to position $\mathrm{p}$ of $A^{\prime}$ is applied to the feature vector of position q in $B^{\prime}$. 


\subsection{Progressively Variant Texture Synthesis Methods}

We have proposed a texture synthesis process through texture blending and the outputs have progressively variant textures. In this section we are mentioning algorithms which generates progressively variant textures.

There is an algorithm for synthesis of progressively variant textures on arbitrary surfaces where the authors used the feature-based blending technique [42]. The technique can create a smooth transition between two given homogeneous textures. There are progressive changes of both shapes and colors of texture elements. While synthesizing textures over the surfaces, the synthesized texture elements tend to break apart as they progressively vary. They used the texton mask to address this issue. Texton mask marks the most prominent texture elements in the 2D texture sample. They used the Gaussian blurring to smooth out the discontinuities inherited from both of the input textures.

Li-Yi Wei generated an algorithm of texture synthesis by fixed neighborhood searching [40]. In this algorithm the texture mixture of multiple sources is done. There is the transition region in the output after mixing where there is a change of texture from one input to other. Weight is assigned for each source. Those weights are the user selected parameters specifying how the input sources should be mixed together. The method generates the Gaussian pyramids of each input and constructs the output pyramid. At each level of the Gaussian pyramids, histogram equalization is done. The method determines the output pixel value which preserves local similarity simultaneously with all the input sources. Fig. 2.1 shows the result

from synthesis of Progressively-Variant Textures on Arbitrary Surfaces [42] and Wei's method [40].

In addition to Wei's texture mixture algorithm, there are other texture mixture approaches but those methods often focus on mixing the texture elements instead of their progressive variation. Some algorithms consider progressive variation while regenerating or expanding of single texture. There is an exemplar-based algorithm of non-stationary texture synthesis by adversarial expansion [44]. The algorithm 


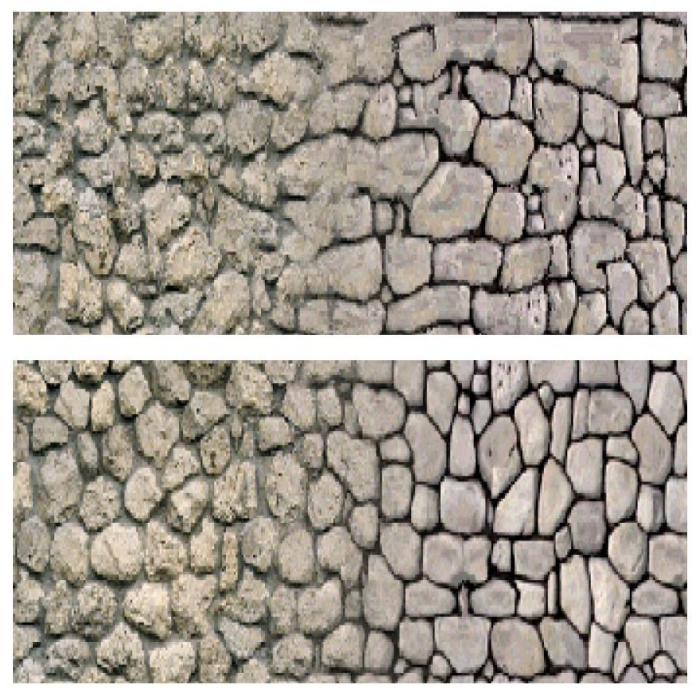

Figure 2.1: The result of synthesis of Progressively-Variant Textures on Arbitrary Surfaces [42] (top) and result from Wei's method [40](bottom).

synthesizes non-stationary, inhomogeneous textures using generative adversarial network. The generative adversarial network is trained to double the spatial extent of texture blocks extracted from a specific texture exemplar. This approach also supports texture transfer but it occurs for regeneration or expansion of single input texture. There is an algorithm of synthesis of inhomogeneous texture where the algorithm generates a source guidance map from an input exemplar [43]. This map enables controlling of both spatial progression and local orientation across the synthesized textures.

\subsection{Relevance Of Previous Methods To Our Algo- rithm}

In this and in the following paragraphs of this section we are describing some of the ideas as well as some libraries that we have used in our algorithm. Heeger and Bergen [21] performed histogram matching of input texture on a uniform white noise. They generated image pyramids and at each level of the pyramids, they performed the histogram matching. Fig 2.2 shows the result of the texture synthesis algorithm by Heeger and Bergen. In our algorithm we generated Laplacian pyramids 

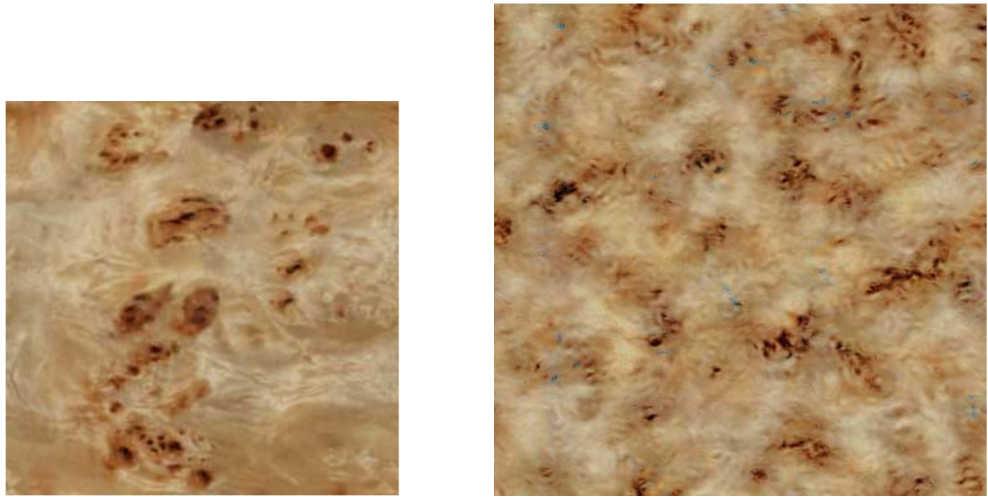

Figure 2.2: The result of texture synthesis by Heeger and Bergen. The process synthesizes by histogram matching of the input, applying on uniform white noise and using image pyramid [21].

of the input images and at each level of the Laplacian pyramids, we used coefficients blending using smooth maximum function.

There is an algorithm of augmenting photographs with textures [12]. In that algorithm the authors do coefficient mixing with smooth maximum function with a view to stylize photographs with auxiliary textures. We used that idea of coefficient blending using the smooth maximum function where we can do weighted blending. We use weighted smooth maximum function where we can control the output of the function based on some of our chosen blending factors.

We generated some of our input images using the histogram preserving blending operator proposed by Eric Heitz and Fabrice Neyret [22]. In the histogram preserving blending algorithm [22], the authors generated a color look-up table using Fast Transport Library. In our algorithm we used the same library for generating the color look-up table and for assigning color to our greyscale output obtained after coefficient mixing. By generating the look-up table and by assigning colors from it, the actual colors of the input images are preserved in our algorithm.

Our algorithm is a new approach of texture synthesis by blending two input textures using the Laplacian pyramid, smooth maximum function, and the SLIC segmentation. In our approach, we regenerated texture through the process of texture blending of two textures using the coefficient mixing at the levels of the 
Laplacian pyramids. In the algorithm of synthesis of progressively variant textures on arbitrary surfaces [42], the authors generated progressively variant texture in the output. Our algorithm also produces progressively variant textures but in our results we have gradual progression as well as some irregular scattered distribution of the textures. This scattered distribution in the transition region in the output, gives a natural merged appearance. Due to this scattered and fuzzy mixture of both the textures, there is no situation of sudden change or breaking down of the textures as they progressively vary. In the algorithm by Jingdan et al [42], the authors did Gaussian blurring to smooth out the discontinuities or the broken regions.

In the following chapters we proceed towards describing our algorithm. In the third chapter we describe the histogram preserving algorithm [22] in detail since we reimplemented the algorithm and used some of its output as the inputs of our algorithm. We used the same library used in the histogram preserving algorithm for generating the look-up table for assigning colors. In the fourth chapter we mention the algorithm and theories we use in our algorithm. In fifth chapter we describe the steps of our algorithm. 


\section{Chapter 3}

\section{Background}

In this chapter, we are going to describe all the algorithms and theories based on which we generated our algorithm. We are describing each of them in separate sections. We described the histogram-preserving blending algorithm step by step because we re-implemented it and used some of the generated results as the inputs of our algorithm.

\subsection{Random Phase Texture}

We started this project focusing mainly on the random phase textures. These are the stochastic micro-textures which are perceptually invariant to phase randomization of their Fourier transform. There are no well-defined visual objects or structures in the micro-textures. Their perceptual characteristics are concentrated on fine non-salient details. These textures are only described by the statistical characteristics. The phase of an image mainly contains the geometric contours of the image and the modulus contains the texture. [11]

Lewis first introduced the idea of using a random phase to obtain a texture from a given Fourier spectrum [31]. The random phase noise (RPN) associated with a discrete image $\hat{h} \in \in^{M \times N}$ is a random real image having a random phase but it has the same Fourier modulus as $\hat{h}$ [31]. If $\mathrm{Z}$ is a random image then it will be a random phase noise associated with $h$ if there exists a uniform random phase $\theta$ such that

$$
\hat{Z}(\xi)=\hat{h}(\xi) e^{i \theta(\xi)}, \xi \in \Omega
$$


If a real-valued image has a phase of $\phi$ and $\theta$ is the uniform random phase then the random image $(\theta+\phi) \bmod 2 \pi$ is also a uniform random phase. Therefore, the RPN associated with an image h only depends on the Fourier modulus of the image [17].

\subsection{Histogram-Preserving Blending Operator}

In this section, we are describing histogram-preserving blending algorithm in details. We re-implemented it as a part of our thesis and used some of the generated results as the inputs of our algorithm.

Non-Gaussian input means the pixel values of the image do not follow Gaussian distribution. In this paper the term "Gaussianization" is used. It means transforming the pixel values so that the histogram of the image has the Gaussian distribution. Eric Heitz and Fabrice Neyret proposed a new by-example noise algorithm using a histogram preserving blending operator [22]. They applied the histogram preserving blending on non-Gaussian inputs. The inputs were first gaussianized with histogram transformation. Then the blending algorithm was applied. Then de-gaussianization was done by the inverse transformation.

The main goals of the histogram preserving texture synthesis through by-example random phase noise synthesis are increased speed and wide generative space. Their method is 20 times faster than Texton Noise [16] which is one of the fast algorithm for random phase texture generation. Eric Heitz's and Fabrice Neyret's method also works for a wide set of non-random phase inputs. This method works well on non-periodic and stochastic looking inputs without requiring any tuning afterwards and without pre-setting any parameters by the user. There are no visual artifacts such as ghosting, softened discontinuities, reduced contrast and new colors which were not in the inputs. These problems were analyzed in their work. The authors also identified how linear blending affects the original histogram of the input.

The new blending operator preserves the histogram and preserves the mean and the variance of the input texture. To preserve the structure of the input a method was designed which fetch and blends the input only 3 times. The fewer patches are blended the more their structure is preserved. It was shown how to precompute and 
store the histogram transformation such that the algorithm can be implemented in a fragment shader. The pixels are blended inside a fragment shader such that it's output directly belongs to the right histogram without requiring any further post-process. It does not produce any grid revealing contrast variations.

The process takes a small stochastic texture as input. Applying the algorithm on the input, a larger output with the same appearances as the input is generated. Any kind of random-phase textures and some irregular, non-periodic, non-random-phase textures are taken as inputs. The same input texture is generated as the output but with larger size. The methodology mainly divides the output space into triangle grid and associate each vertex with a random patch from the input texture. Inside the triangle, 3 random patches from the input is blended. This blending is done in a way so that the histogram of the input texture is preserved in the output.

At the first step of the algorithm, random variables from a Gaussian distribution were generated. The three dimensional Gaussian distribution was generated in each direction so that random values fit well on interval $[0,1]$ with 8 -bit precision. For each RGB component, a uniform random number $U \in[0,1]$ was generated. The inverse of the Cumulative Distribution Function(CDF) was applied on these uniform numbers. This function converts the uniform values to random Gaussian variables.

$$
G=\frac{1}{2}+\sigma \sqrt{2} \operatorname{erf}^{-1}(2 U-1)
$$

Where erf is the error function and $\operatorname{erf}(z)$ is defined by

$$
\operatorname{erf}(z)=\int_{-\infty}^{z} \frac{1}{\sqrt{2 \pi}} e^{\frac{-z^{2}}{2}} d z
$$

In Eqn. 3.1, $\sigma$ is the variance. Through this equation an image of random Gaussian variables, $G_{k, l}$ of same size as the input image is obtained. Here $(k, l)$ denotes the pixel co-ordinates. The Gaussian version of the image must have the same recognizable structure as in the input. For this purpose, the mapping of the random Gaussian variables with the input pixel values was done using Optimal Transport solver(OT solver) [5]. 
To create the gaussianized version of the input image, the Gaussian variables were mapped with the input image using the OT solver. It is the Fast Transport Library developed by Bonneel [5]. The best permutation of the pixels to attain the structure of the input image was done by the Lagrangian OT solver. The library does the mapping on the basis of the minimum error on L-2 norm Earth Mover's Distance (EMD) between the input image and the image of random Gaussian variables. The distance is calculated based on both the color matching and finding position of each color pixel in the output so that structures in the input image are obtained. This re-arrangement of pixels is done with the minimum error (Eqn. 3.3) to achieve the structure in input.

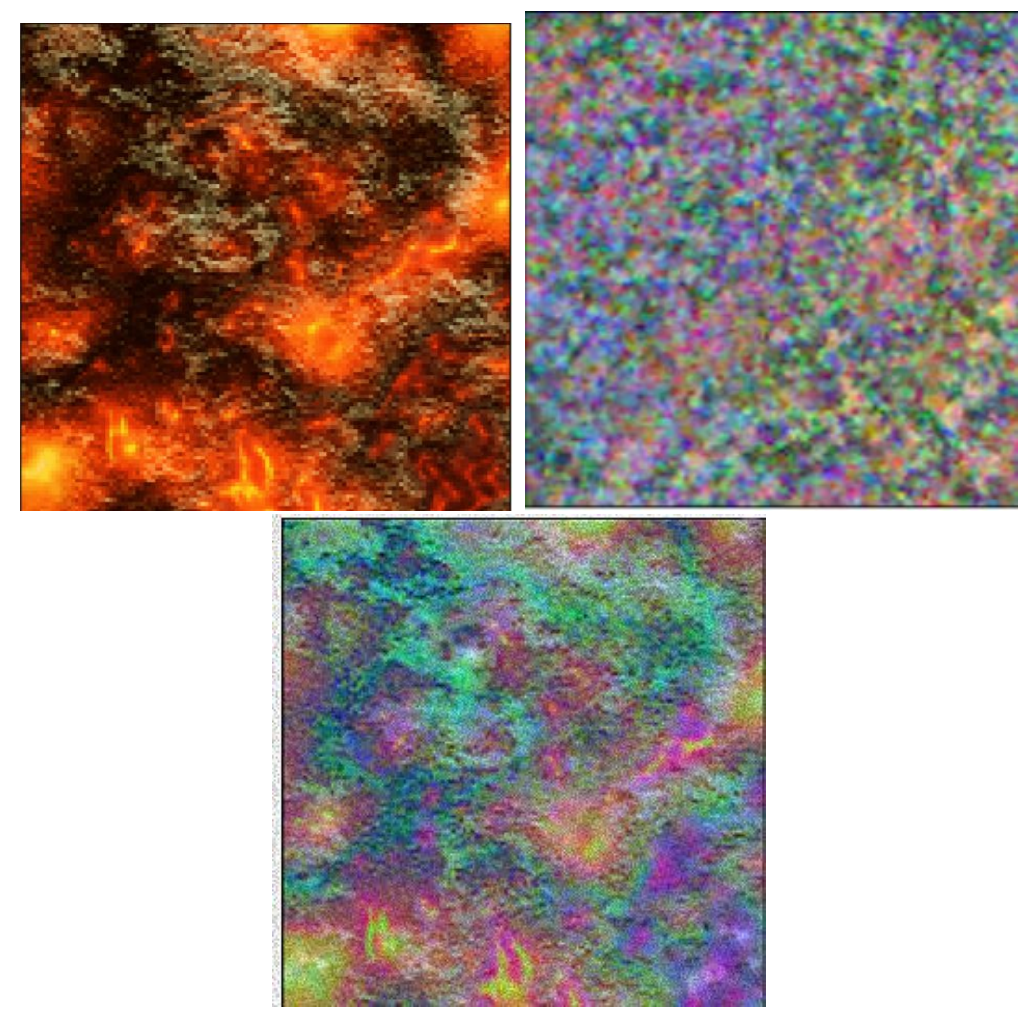

Figure 3.1: By mapping the random RGB values(upper right image) with the input image(upper left image) using the OT solver, the image with the same structure as the input is obtained (the bottom image)

Let pixel values of the input image are noted as $X_{i, j}$ where $(i, j)$ is pixel coordinates. Let its histogram is denoted by $\mathcal{H}$. $\mathcal{T}$ is the transformation of the image. This transformation means gaussianization of the image. The pixels of the gaussianized 
image is represented as $G_{k, l}$. The gaussianized image has the same structure as the input. This transformation, $\mathcal{T}$ gaussianizes the image with minimum $L^{2}$ error :

$$
\text { error }=\min _{\mathcal{T}} \sum_{i, j}\left(G_{k, l=\mathcal{T}(i, j)}-X_{i, j}\right)^{2}
$$

Minimizing the error in Eqn. 3.3 means finding a one-to-one mapping $(k, l)=$ $\mathcal{T}(i, j)$ that minimizes the sum of the squared distances between the pixel pairs of $X_{i, j}$ and $G_{k, l}$. Here the color distance and spatial distance of the corresponding pixels are measured. The image produced at this point has a Gaussian histogram G. In this image, the structures of the input is preserved as much as possible.

\subsubsection{Tiling Of Input Image And Blending Of Patches From Input}

Hexagonal patches from the Gaussianized image are chosen randomly for blending in the output image space. Hexagonal tiles with radius equal to approximately half of the size of the input plane is used. In the output image space, the hexagonal patches are placed in such a way so that regular triangles are formed by overlapping the hexagons. The blending of patches from the input Gaussianized image, $G$ is done in such a way so that the blending of exactly three patches from $G$ occurs inside the triangle. Fig. 3.2 provides an overview of this method.
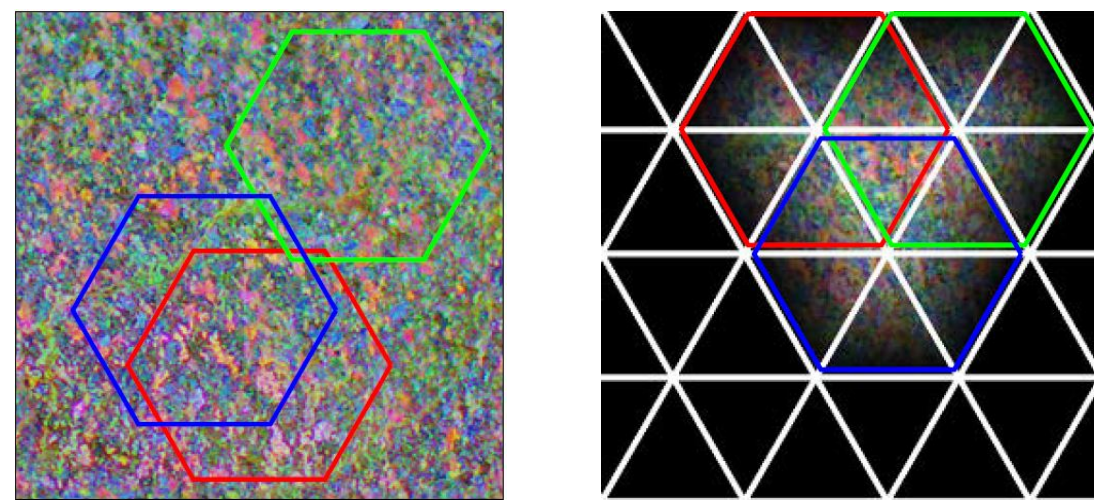

Figure 3.2: Hexagonal patches are chosen from any random locations in the gaussianized input(left). In the output plane (right), inside each triangle formed by intersecting 3 hexagons, 3 patches from the input are blended [22]. 
The Histogram preserving blending operator [Heitz et al. 2018] preserves the Gaussian distribution of G. Therefore, it also preserves the mean and the variance of the input texture.

Let $X_{1}, \ldots, X_{N}$ are $\mathrm{N}$ multivariate random variables that are independent and identically distributed in histogram $\mathcal{H}$. Let $E[X]$, and $\operatorname{cov}[X]$ are expectation and covariance of these random variables. $\mathrm{N}$ non-negative weights $w_{1}, \ldots, w_{N}$ are considered such that their sum is normalized.

$$
\sum_{n=1}^{N} w_{n}=1
$$

When 3 regular hexagons overlap in the output space then an equilateral triangle is formed. The vertices of the triangle are the center of the hexagons. For each of the RGB value, $X_{n}$ inside the triangle, the weight $w_{n}$ of each of the vertices of the triangle is calculated. The larger the weight the more the variable should contribute to the result. A variable should not impact the result at all if it's weight is 0 . $W$ is the variance factor and it calculated by

$$
W=\sqrt{\sum_{n=1}^{N} w_{n}^{2}}
$$

The histogram preserving blending operator generated by Heitz and Neyret [22] works in the following way

$$
X^{\text {final }}=\mathcal{T}^{-1}\left(\frac{\sum_{n=1}^{N} w_{n} \mathcal{T}\left(X_{n}\right) E[G]}{W}+E[G]\right)
$$

Here, $\mathcal{T}$ is the histogram transform, it's inverse is $\mathcal{T}^{-1}, E[G]$ is the expectation of the random variables with Gaussian distribution and $W$ is the variance factor. For each of the RGB value, $X_{n}$ inside the triangle, the weight $w_{n}$ of each of the vertices of the triangle is calculated. The weights for three vertices of a triangle are barycentric co-ordinates.

Here, $d_{a}, d_{b}$ and $d_{c}$ are linear distance of pixel, $\mathrm{P}$ from vertices $\mathrm{A}, \mathrm{B}, \mathrm{C}$ respectively. Therefore, for vertices $\mathrm{A}, \mathrm{B}$ and $\mathrm{C}$, we calculate barycentric co-ordinates $w_{A}, w_{B}$ and 


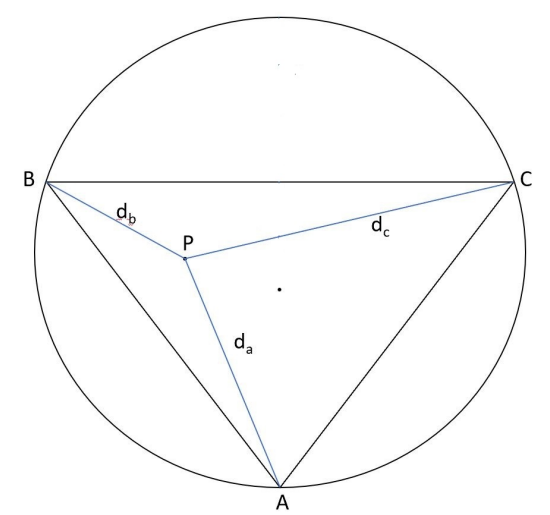

Figure 3.3: In the blending equation, the barycentric co-ordinates $w_{A}, w_{B}$ and $w_{C}$ are determined for vertices $\mathrm{A}, \mathrm{B}, \mathrm{C}$ respectively

$w_{C}$. The value of these weights are applied in histogram preserving blending operation in Eqn. 3.6.

\subsubsection{Inverse Transformation}

At this point, an image of blended Gaussian values, $G_{i, j}^{c o v}$ is obtained. To get back the original image but of extended size having the same histogram, inverse histogram $\mathcal{T}^{-1}$ transformation is done. The blended Gaussian values, $G_{i, j}^{\text {cov }}$ are not the same as $G_{i, j}$. They are statistically distributed in the same Gaussian distribution but they are not exactly the same point set. That is why OT solver is used second time on another point set to compute the inverse transformation. This can be done directly using Eqn. (3.3) i.e. by mapping between blended Gaussian values to RGB values chosen randomly from the input image. But it is not suitable for real-time rendering because the run time is very long. In order to do it efficiently at run time the mapping is precomputed and stored in a three dimensional look-up table that can be fetched at run-time. A $32^{3}$ look-up table is used. The three dimensional Gaussian values from the blended Gaussianized image was mapped with $32^{3}$ or 32,768 pixels from the input image. Those mapped pixels from input are stored in the $32^{3}$ look-up table. The look-up table is a $32 \times 32 \times 32$ array which stores the 32,768 number of mapped pixels from input. Thus a point set of Gaussian random points in RGB space is mapped to $32^{3}$ number of pixel values chosen randomly from the input image. Those pixels from the input image is stored in cells of the look-up table. The Gaussian histogram is spread uniformly over 32,768 cells. Through inverse transform parameterization, 
uniform values are obtained and those are linearly interpolated to find a location in the look-up table.

$$
G=\frac{1}{2}+\sigma \sqrt{2} \operatorname{erf}^{-1}(2 U-1)
$$

Inverse parameterization:

$$
U=\frac{1}{2}+\frac{1}{2} \operatorname{erf}\left(\frac{G^{c o v}-\frac{1}{2}}{\sigma \sqrt{2}}\right)
$$

At runtime, the blended Gaussian value $G^{\text {cov }}$ is used to calculate U and fetch the look-up table at the three dimension location $\mathrm{U}$, where the mapped pixel from the input is stored. In the original paper [Heitz et al. 2018] this algorithm was implemented in GPU and the blending operation was done inside a fragment shader.

\subsubsection{Results Of Histogram-Preserving Algorithm}

We re-implemented the histogram-preserving blending algorithm generated by Heitz and Neyret and got the following results.

On the output in middle row in Fig. 3.4, some sort of irregular repetitive patterns can be observed. These repetition became prominent due to presence of black patches in output. In the output of texture in the last row of Fig. 3.4, the repetitions are noticeable due to the presence of some black pebbles or black holes. In the output of texture on the first row in Fig. 3.4, there are more uniform regions than the ridged parts of input textures. There are no wrong colors in the outputs since all the colors which are assigned are from the inputs. There is no ghosting in the outputs. One interesting thing which can be observed for input texture in the last row in Fig. 3.4 is, most of the shapes of tiny pebbles are preserved. Some tiny and large pebble shapes got totally distorted.

The mapping by the optimal transport solver twice in the whole process takes significant amount of runtime. That is why, for efficient use of the inverse mapping, the inverse transformation is precomputed and stored in a three dimensional look-up table. 

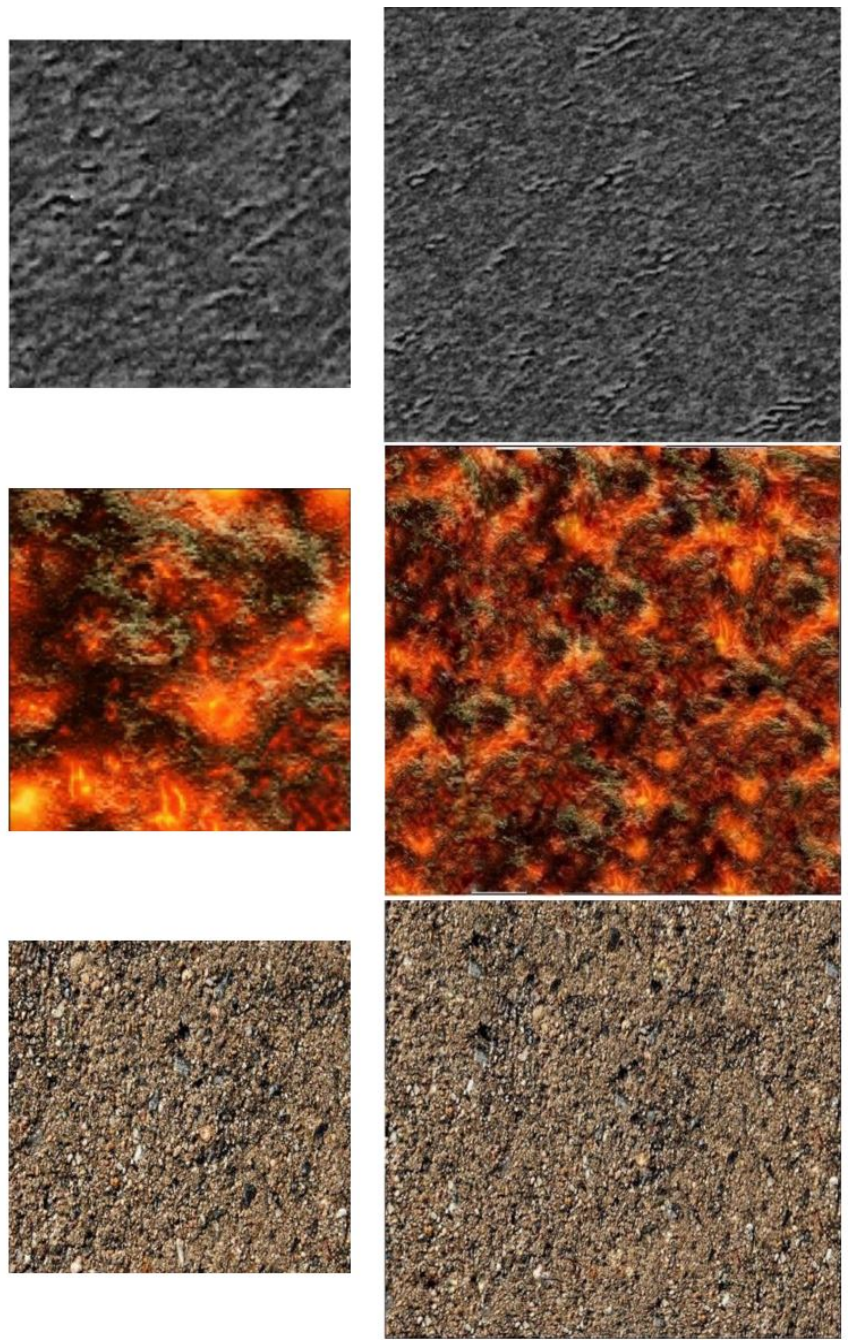

Inputs

Outputs

Figure 3.4: Results of Histogram preserving blending algorithm 
We used some of the outputs generated by the histogram-preserving blending operator as the inputs of our algorithm. There is no definite reason for taking these results as our input. We can use other inputs as well for our algorithm. We used the same OT solver, Fast Transport library developed by Bonneel [5], that is used in this histogram-preserving algorithm.

\subsection{Laplacian Pyramid}

A Gaussian pyramid is built by repeatedly downsampling the original image. Then the Laplacian pyramid is constructed by calculating the difference between the image on level L of the Gaussian pyramid and the upsampled version of the one at level $\mathrm{L}+1$. Each error image resulting out of this difference is a level in the Laplacian pyramid. Compression is achieved by quantizing the pixel values in the error images. By upsampling and summing all the levels of the Laplacian pyramid the original image can be recovered [7].

We used Laplacian pyramid in our algorithm of texture synthesis. We generated Laplacian pyramid of the input images for accessing the coefficients of pixel values at each level of the pyramid. We perform coefficient mixing of the two inputs at each level of Laplacian pyramid. We shall discuss the details in our algorithm section.

\subsection{Smooth Maximum Function}

We generate two Laplacian pyramids derived from two inputs and we compute a result pyramid that combines coefficients from both inputs. We perform the coefficient mixing using the smooth maximum function [9]. Smooth maximum function generates the output which is greater than the maximum between the two coefficients. We generate a parameter that can control how much the output will be greater than the maximum coefficient. That is why we use the smooth maximum function instead of the general maximum function,

$$
f(x, y)=\max (x, y)
$$

The smooth maximum of two variables $\mathrm{x}, \mathrm{y}$ is the function 


$$
g(x, y)=\log (\exp (x)+\exp (y))
$$

The smooth maximum approximates the general maximum function, Eqn. 3.9. If $\mathrm{x}$ is a little bigger than $\mathrm{y}, \exp (\mathrm{x})$ will be a lot bigger than $\exp (\mathrm{y})$. Exponentiation exaggerates the difference between $\mathrm{x}$ and $\mathrm{y}$. If $\mathrm{x}$ is significantly bigger than $\mathrm{y}, \exp (\mathrm{x})$ will be so much bigger than $\exp (\mathrm{y})$ that $\exp (\mathrm{x})+\exp (\mathrm{y})$ will essentially be equal to $\exp (\mathrm{x})$ and the smooth maximum will be approximately $\log (\exp (\mathrm{x}))=\mathrm{x}$, the general maximum [9].

If $\mathrm{x}$ and $\mathrm{y}$ are multiplied by a large constant, the smooth maximum will be closer to the general maximum when divided by the constant. For example, $\mathrm{g}(1,2)=2.31$, but $\mathrm{g}(10,20)=20.00004$. So if we divide the result by the constant, 10 we get 2.000004 and $2.000004 \approx 2$. Thus we can control the output of the smooth maximum by generalizing the smooth maximum to be dependent on a parameter $\mathrm{k}$ [9].

$$
g(x, y ; k)=\log (\exp (k x)+\exp (k y)) / k
$$

We can make the smooth maximum as close to the general maximum by making $\mathrm{k}$ large enough.

\subsection{SLIC Superpixel}

Simple Linear Iterative Clustering (SLIC) is an algorithm for superpixel generation. Superpixel is a group pixels which is perceptually meaningful atomic regions, which can be used to replace the rigid structure of the pixel grid [1]. The SLIC algorithm takes the required number of superpixels, $K$ as input. The superpixels are approximately of equal size. $K$ number of superpixel cluster centers or centroids, $C_{k}$ are chosen with $k=[1, K]$ at regular grid intervals $S$. Spatial range of any superpixel is approximately $S^{2}$ which is the approximate area of a super-pixel. Therefore, the pixels that are associated with this cluster center assumed to lie within a $2 S \times 2 S$ area around the superpixel center on the xy plane. In the five dimensional space, the normalized distance measure, $D_{s}$ is the sum of the rgb color distance and xy plane distance normalized by the grid interval S. $D_{s}$ is calculated by:

$$
D_{s}=d_{r g b}+\left(\frac{m}{S}\right) * d_{x y}
$$


where $d_{r g b}=\sqrt{\left(\left(r_{k}-r_{i}\right)^{2}+\left(g_{k}-g_{i}\right)^{2}+\left(b_{k}-b_{i}\right)^{2}\right)}$ and

$$
\begin{aligned}
& d_{r g b}^{A^{\prime}}=\sqrt{\left(\left(r_{p}^{A^{\prime}}-r_{c}^{A^{\prime}}\right)^{2}+\left(g_{p}^{A^{\prime}}-g_{c}^{A^{\prime}}\right)^{2}+\left(b_{p}^{A^{\prime}}-b_{c}^{A^{\prime}}\right)^{2}\right)} \\
& d_{r g b}^{B^{\prime}}=\sqrt{\left(\left(r_{p}^{B^{\prime}}-r_{c}^{B^{\prime}}\right)^{2}+\left(g_{p}^{B^{\prime}}-g_{c}^{B^{\prime}}\right)^{2}+\left(b_{p}^{B^{\prime}}-b_{c}^{B^{\prime}}\right)^{2}\right)}
\end{aligned}
$$

$d_{x y}=\sqrt{\left(\left(x_{k}-x_{i}\right)^{2}+\left(y_{k}-y_{i}\right)^{2}\right.}$ and the variable $m$ is used to determine the compactness of a superpixel. The compactness of a superpixel will be more with the increase in the value of $\mathrm{m}$. In order to avoid placing the centroids at an edge and to reduce the possibility of choosing a noisy pixel, sampling of $\mathrm{K}$ regularly spaced centroid is done. The centroids are moved to seed locations where there are the lowest gradient in the $3 \times 3$ neighborhood. The gradients are computed as:

$$
G(x, y)=\|I(x+1, y)-I(x-1, y)\|^{2}+\|I(x, y+1)-I(x, y-1)\|^{2}
$$

Here, $I(x, y)$ is the rgb vector corresponding to the pixel at position $(\mathrm{x}, \mathrm{y})$ and $\|$.$\| is the L 2$ norm.

We used the SLIC segmentation at an intermediate step in our algorithm. We segmented the output image space with the SLIC and performed our coefficient merging at each segment separately. The effect of this segmentation will be discussed in the next chapter. 


\section{Chapter 4}

\section{Random Phase Texture Transition With Smooth Maximum}

We blended the coefficients in the Laplacian pyramids of the inputs using the smooth maximum function. The coefficients in the Laplacian pyramid of an image represent essentially the local changes in the image content by giving the edge information of the image. Therefore, when we blend the coefficients of the pyramid of both images, we can blend the structures of the texture contents of both inputs. Furthermore, when we are using the weighted smooth maximum function for blending, we calculate the weights based on some of our chosen blending factors. Based on the value of the weights, we achieved our desired blending and distribution of the textures in the output. Since, the smooth maximum function generates the output which is almost equal to the higher input coefficient, we can preserve the most prominent structures in the textures.

In this chapter, we are describing the different steps of our algorithm in each sections. At the first section of this chapter, we shall mention the goal of our experiment and different steps of our algorithm briefly. Then at the following sections we are going to describe each of our steps in detail and the reasons for each step.

\subsection{Pipeline Of The Steps Of Our Algorithm}

Let $A$ and $B$ are two random phase input textures and $A^{\prime}$ and $B^{\prime}$ are the outputs of the histogram preserving blending algorithm [22]. We take $A^{\prime}$ and $B^{\prime}$ as our input. 
Inputs $A$ and $B$ can also be used directly without using the histogram preserving blending algorithm.

One important parameter in our algorithm is the measure of the proportion of texture contents in the output from both inputs. This measure can be based on any factor like local contrast, correlation, time. In our experiment the measure of the texture contents from each input and the distribution of textures are based on the spatial location of each pixel in the output. We calculate the linear distance of the pixels from left edge of the output image and denote it by $t$. But this $t$ can be derived based on any other factors like local contrast, color and in any scale other than only linear distance. We name this parameter $t$ as the blend factor.

We considered the linear distances of the pixels along $\mathrm{x}$-axis because we want the texture transition from the left edge to the right edge of the output image. We consider $A^{\prime}$ as the left texture and $B^{\prime}$ as the right texture. The goal is to merge input $A^{\prime}$ and $B^{\prime}$ such that the generated output will demonstrate a smooth texture transition from $A^{\prime}$ textures to $B^{\prime}$ textures. Towards the left region of the output there will be textures from $A^{\prime}$ and towards the right region of the output there will be textures from $B^{\prime}$. Within the middle region of the output, there will be a gradual progression of textures from $A^{\prime}$ to textures from $B^{\prime}$. An example in Fig. 4.1 explains the transition of textures.

In Fig. 4.1, the grass image is considered as the left input and the pebbles image is considered as the right input. Therefore, towards the left region of the merged output there are mostly grass textures and towards the right region there are mostly pebbles textures. Within the middle area, texture is synthesized in such a way that there is a continuous progression from the grass texture to the pebbles texture.

Another factor based on which we calculate the weights for the smooth maximum function is the "textureness" of the inputs. Textureness denotes local high frequency content in the image [4].

We are going to briefly mention each step of our algorithm in this section.:

Step1:We take two image inputs, $A$ and $B$. From the histogram preserving 


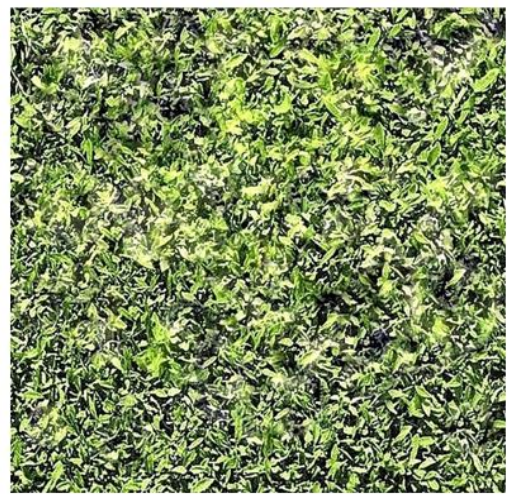

Grass Input (left texture)

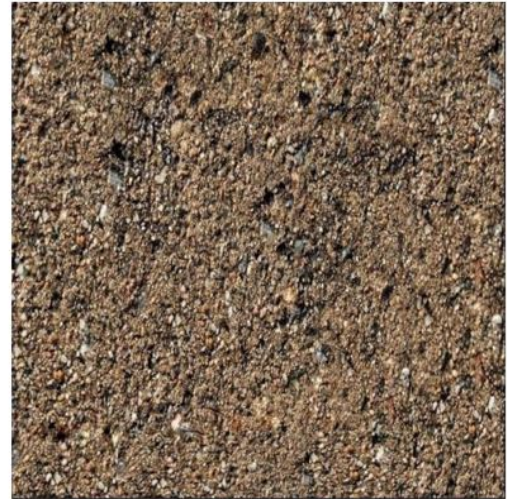

Pebbles Input(right texture)

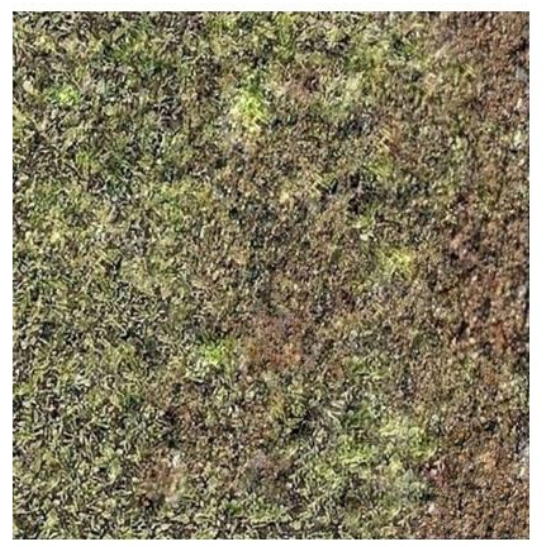

Merged Output

Figure 4.1: The generated output of our algorithm which demonstrates a gradual transition of textures from the left image to textures from the right image

blending algorithm we generate the outputs of required size $A^{\prime}$ and $B^{\prime}$ respectively.

Step2:We create a segmentation map for the output where there will be the blended coefficients. The same segmentation map is created by joint SLIC algorithm where two color distances are measured.That means, while generating the SLIC segments, the color distances of two pixels at same location in both $A^{\prime}$ and $B^{\prime}$ from their corresponding centroids are considered. For each segment in the input we generate two Laplacian pyramids $L^{A^{\prime}}$ and $L^{B^{\prime}}$ for $A^{\prime}$ and $B^{\prime}$ respectively. At each level of the pyramids, we blend the coefficients. For a given segment, the coefficients which are within the area of that segment in both inputs are blended. This is done for all the segments obtained using the SLIC. We use SLIC segmentation in order to preserve the actual textures from each input after the blending. 
Step3: We find the Root Mean Square(rms) of the coefficients, $g_{l}$ and $h_{l}$ of both inputs respectively at each level, $l$ of the Laplacian pyramid. The rms is measured to estimate the textureness of the inputs. $t$ denotes the measure of proportion of texture contents in the output. For each SLIC segment, at each level, $l$ of the Laplacian pyramids, we calculate the weights $p_{l}^{\prime}$ and $q_{l}^{\prime}$. We calculate the weights based on the value of parameter $t$ and the rms values, $g_{l}$ and $h_{l}$. We apply the weights on coefficients from each input while blending the coefficients. For blending the coefficients we use the smooth maximum function. By changing the values of the weights, $p_{l}^{\prime}$ and $q_{l}^{\prime}$ we can control the output of the smooth maximum function.

Step4: The parameter $t$ is determined for the coefficient at the centroid of each of the SLIC segment. Then we apply the same value of $t$ in the smooth maximum function for all the coefficients from both inputs within the area of the segment. This is done to make the outputs of the smooth maximum function to be close to the values of any one input coefficients only. When the majority of the output coefficients are close to the coefficients of a particular input then the original textures from that input are preserved.

Step5: At this step we generate $R$ number of random numbers, $r_{(1 . . R)}$ and choose $\mathrm{R}$ number of random SLIC segments, $\operatorname{seg}_{(1 . . R)}$. If a random number, $r_{R}$ is greater than $t$ of the centroid of a segment, $\operatorname{seg}_{R}$ then we put more weight on one of the two coefficients in smooth maximum function. If the random number is smaller than $t$ of the centroid, then we put more weight on the other coefficient in the smooth maximum function. This step contributes in giving the output a scattered and realistic look.

Step6: For each of the SLIC segment, we get a resultant pyramid, $L^{\prime}$ with blended coefficients. Combining the levels of resultant pyramid we get a greyscale blended segment. If there are $K$ number of segments then we get $K$ blended segments. All the blended segments together constitute the output greyscale image.

Step7: We assign color to the greyscale image from either one of the two precomputed lookup tables of $A^{\prime}$ and $B^{\prime}$. Color from which lookup table to choose 
from is decided by the maximum number of times the output coefficient is close to coefficient of either $A^{\prime}$ or $B^{\prime}$ in the levels of the pyramid.

Step8: After assigning colors there might be some discontinuities along the boundaries. Discontinuities occur when textures from $A^{\prime}$ and $B^{\prime}$ are side by side and there is a drastic change of color in texture contents from $A^{\prime}$ and $B^{\prime}$. For smooth merging around the boundaries, the alpha blending of color is done for some rows and columns consisting the boundary pixels. Those rows and columns containing the boundary pixels are named as halos. We make a smooth transition of color from one texture content to another along the SLIC segments boundaries.

Now we are going to explain the steps of the algorithms in detail and the reasons for each step.

\subsection{Algorithm}

In this section we are giving a detailed explanation of each of the steps of our algorithm. We first describe the coefficient blending in the Laplacian pyramid without using the SLIC segmentation. Then we describe the blending using SLIC segmentation and describe how the SLIC segmentation improves the results.

\subsubsection{Blending Coefficients}

We blend the coefficients in the Laplacian pyramid using the smooth maximum function. We use weighted smooth maximum function so that we can control the outputs of the function according to some factors. We will describe the factors and also describe how the required outputs are generated based on the factors.

$A^{\prime}$ and $B^{\prime}$ are two output images from histogram preserving blending algorithm. We will use these two images as the inputs of our algorithm. We can also use two images $A$ and $B$ directly in our algorithm without using the histogram preserving algorithm. We are seeking to accomplish seamless texture blending by blending the 
coefficients in the Laplacian pyramid representation of the two input textures.

We generate two Laplacian pyramids $L^{A^{\prime}}$ and $L^{B^{\prime}}$ of inputs $A^{\prime}$ and $B^{\prime}$ respectively. Then we compute a resultant pyramid $L^{R}$. We used a weighted smooth maximum function in our algorithm. We calculate the weights for the coefficients from both inputs based on two factors. First factor is $t$ which denotes the ratio of proportion of textures contents from input A to the proportion of textures contents from input B. Thus $t$ denotes how much of the texture contents from both of the inputs will be preserved in the output.

The second factor is the local textureness of the input images, where "textureness" denotes local high frequency content [4]. In the output there will be textures from both of the inputs depending on textureness of the input.The textureness is estimated by calculating the root mean square (rms) of all the coefficients at a given pyramid level.

In our algorithm, we formulated the smooth maximum function in following way:

$$
S M\left(u_{l}, v_{l}, p_{l}^{\prime}, q_{l}^{\prime}\right)=\frac{1}{\sqrt{p_{l} q_{l}}}\left(\ln \left(e^{p_{l}^{\prime} u_{l}}+e^{q_{l}^{\prime} v_{l}}-1\right)\right)
$$

Here, $l$ denotes the level number of the Laplacian pyramids. At level $l, u_{l}$ and $v_{l}$ are the coefficients from $A^{\prime}$ and $B^{\prime}$ respectively. $A^{\prime}$ and $B^{\prime}$ are the outputs of the histogram preserving blending operation in chapter 3. $p_{l}^{\prime}$ and $q_{l}^{\prime}$ are the weights applied on the coefficients $u_{l}$ from $A^{\prime}$ and $v_{l}$ from $B^{\prime}$ respectively. We subtract one from the sum of the exponentiated inputs. This adjustment ensures that when both of the Laplacian coefficients are zero then the output will also be zero. The notations and their meanings are also shown in the table 5.1.

The output of the smooth maximum function depends on the weights $p_{l}^{\prime}$ and $q_{l}^{\prime}$. Higher rms means higher textureness in the image contents. We generate the weights based on the rms values because we want to ensure that the contents having lower textureness from one input are not overwhelmed by the higher textureness in the 
Table 4.1: Symbol Definitions

\begin{tabular}{l|c} 
Notation & Meaning \\
\hline$l$ & level of the Laplacian pyramids \\
$u_{l}$ & value of a coefficient of the image $A^{\prime}$ at level $l$ \\
$v_{l}$ & value of a coefficient of the image $B^{\prime}$ at level $l$ \\
$p_{l}$ and $q_{l}$ & parameters based on the rms values \\
$g_{l}$ & rms of the coefficients of $A^{\prime}$ at level $l$ \\
$h_{l}$ & rms of the coefficients of $B^{\prime}$ at level $l$ \\
$t$ & the ratio of the proportion of texture contents from both inputs \\
$p_{l}^{\prime}$ & weight applied on $u_{l}$ \\
$q_{l}^{\prime}$ & weight applied on $v_{l}$
\end{tabular}

other input. In effect, we are normalizing the textureness between two potentially dissimilar input textures.

At each level of $L^{A^{\prime}}$, we calculate the rms of the coefficients. We find it for $L^{B^{\prime}}$ as well. Let $g_{l}$ and $h_{l}$ be the rms of pixel coefficients of $A^{\prime}$ and $B^{\prime}$ respectively at the $l^{\text {th }}$ level of the Laplacian pyramid. We calculate the parameters $p_{l}$ and $q_{l}$, based on the rms values in Eqn. 4.3 and Eqn. 4.4.

We compute a target amplitude $a$ in Eqn. 4.2. This target amplitude is a weighted average of the two input rms values, where the weights are computed according to the texture blend factor $t$. We aim to blend the coefficients in such a way that the output coefficients will have a distribution whose rms is near $a$.

$$
a_{l}=(1-t) \cdot g_{l}+t \cdot h_{l}
$$

and we define $p_{l}$ and $q_{l}$ as

$$
\begin{aligned}
p_{l} & =\frac{a_{l}}{g_{l}} \\
q_{l} & =\frac{a_{l}}{h_{l}}
\end{aligned}
$$

We calculate the final weights $p_{l}^{\prime}$ and $q_{l}^{\prime}$ in Eqn. 4.5 and Eqn. 4.6 with the value of $t$. 
Notice that $t$ is in effect included twice: once to compute the target amplitude in Eqn. 4.2 and once to blend between the two coefficients with their magnitudes normalized according to target amplitude, $a$. These weights will be used in the calculation of the weighted smooth maximum to mix two actual coefficients to compute a coefficient for the output pyramid.

$$
\begin{gathered}
p_{l}^{\prime}=p_{l} \cdot(1-t) \\
q_{l}^{\prime}=q_{l} \cdot t
\end{gathered}
$$

Each level of the resultant Laplacian pyramid $L^{R}$ is constructed with the resultant merged coefficients. Using Eqn. 4.1, we compute each output coefficient $L_{l}^{R}(x, y)$ at level $l$ as follows:

$$
L_{l}^{R}(x, y)=\Psi \times S M\left(\left|L_{l}^{A^{\prime}}(x, y)\right|,\left|L_{l}^{B^{\prime}}(x, y)\right|, p_{l}^{\prime}, q_{l}^{\prime}\right)
$$

In the preceding, the variable $\Psi \in\{-1,1\}$ is the sign of the coefficient with the larger absolute value. Combining all the levels of $L^{R}$ when the output image is constructed, a merged image of two texture $A^{\prime}$ and $B^{\prime}$ is formed. Thus we get a greyscale output image having the blended textures from both inputs.

In our experiment we determined parameter $t$ according to the distance measured from the left edge of the output image. We want to blend the coefficients in such a way that the blended coefficients towards the left region of output image are almost equal to the values of coefficients of left input. Similarly the blended coefficients towards the right region of output image are almost equal to the values of coefficients of the right input. By determining $t$ based on the linear distance on the x-dimension of the image plane, we will make a gradual progression of texture contents from both inputs from the left edge to the right edge of the image.

The horizontal length of the output image is normalised to the range of 0 to 1 and $t$ denotes the distance from the left edge of the image. For $t \approx 0$, the output of smooth maximum will approach the value of $u_{l}$. For $t \approx 1$, the output of smooth maximum will approach the value of $v_{l}$. For $t \approx 0.5$, we have formulated Eqn. 4.2 and Eqn. 4.3 in such a way that, coefficients having lower rms value get amplified. 
But irrespective of the value of rms and the value of $t$, if $u_{l} \gg v_{l}$, and thus $p_{l}^{\prime} \cdot u_{l}$ becomes larger than $q_{l}^{\prime} \cdot v_{l}$ then the output value is greater than $u_{l}$. Similarly, if $v_{l} \gg u_{l}$, and thus $q_{l}^{\prime} \cdot v_{l}$ becomes larger than $p_{l}^{\prime} \cdot u_{l}$ then the output value is greater than $v_{l}$.

In the blended greyscale image, there is a gradual transition of textures of $A^{\prime}$ and $B^{\prime}$ from left to the right. In the following paragraph we are describing the effect of the parameters $a_{l}, p_{l}, q_{l}, p_{l}^{\prime}, q_{l}^{\prime}$ and the value of $u$ and $v$ with an example of a sand texture and water wave texture in Fig. 4.3.

In Fig. 4.2, there are two input images of the sand and the water texture. In Fig. 4.3, we show 11 greyscale outputs after converging resultant pyramid levels. We kept fixed value of $t$ throughout the output for explanation. In each of the 11 images in Fig. 4.3, greyscale outputs with 11 constant values of $t$ respectively are shown. In our algorithm, we vary $t$ from 0 to 1 from the left edge to the right edge of the output.
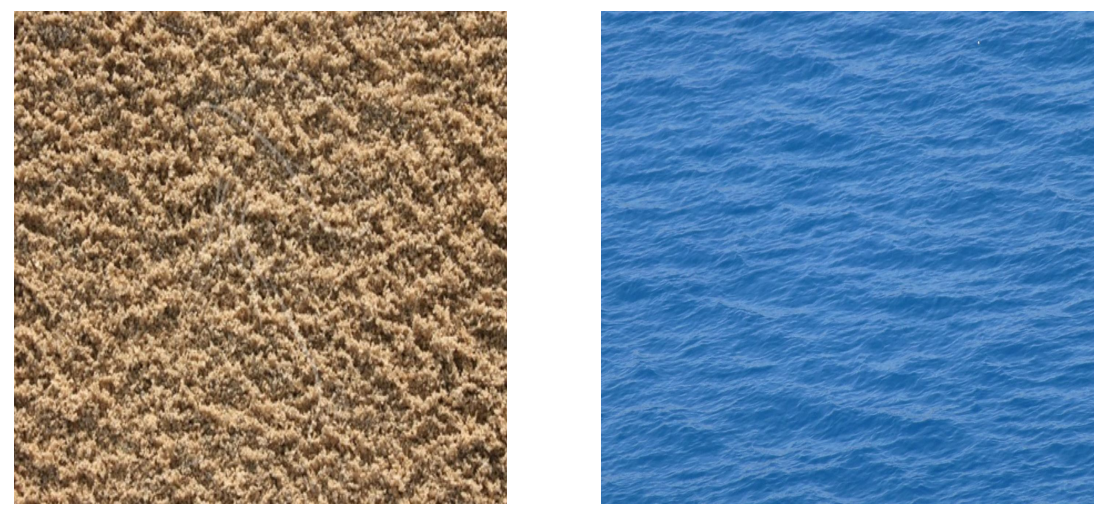

Figure 4.2: Sample inputs for representing the outcomes for different values of $p, q$ and $t$ in smooth maximum function.

Fig.4.3 is the representation of how textures of each input are preserved depending on the spatial location of the coefficients. We can observe as t increases from 0 to 1 , texture contents from sand image to the water image get preserved in the output. When $t=0$ then the output is completely sand texture. When $t=1$, the output is completely water texture. 


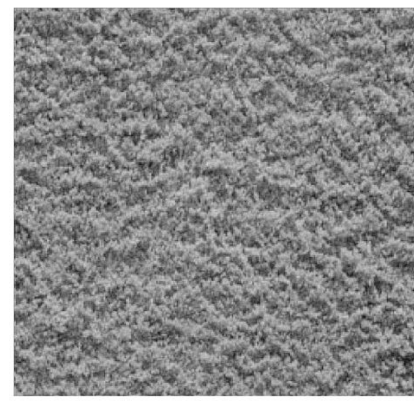

$t=0$

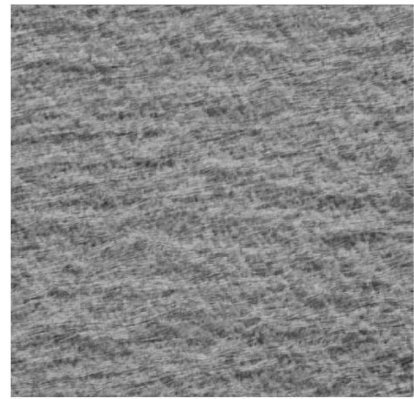

$\mathrm{t}=0.3$

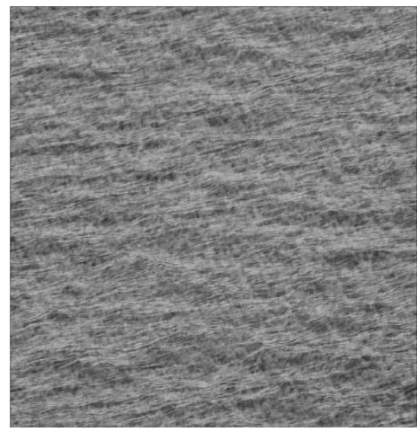

$\mathrm{t}=0.6$

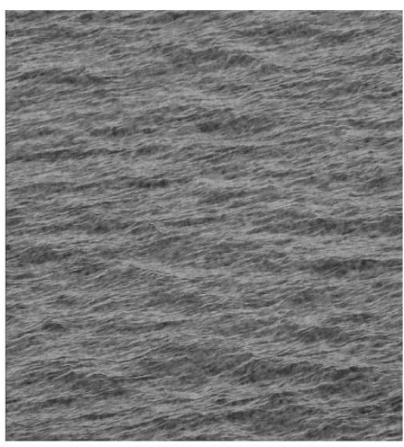

$t=0.9$

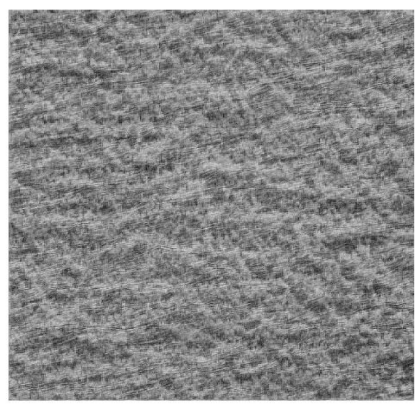

$\mathrm{t}=0.1$

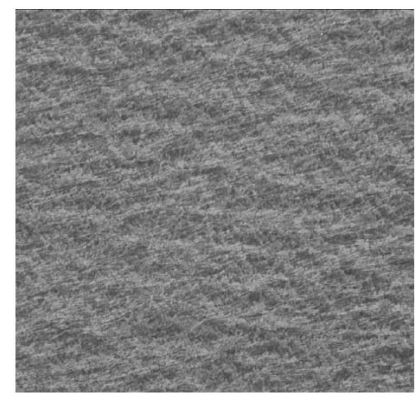

$\mathrm{t}=0.4$

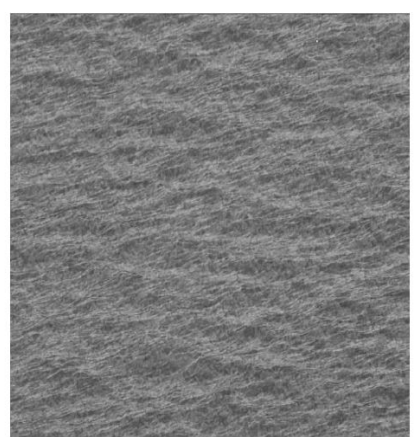

$\mathrm{t}=0.7$

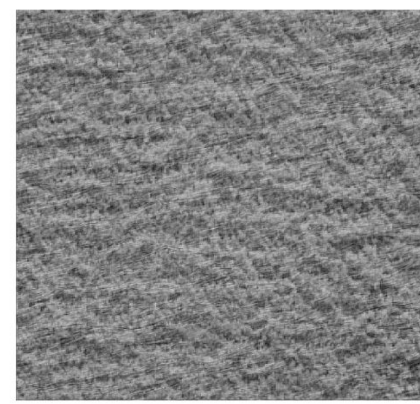

$\mathrm{t}=0.2$

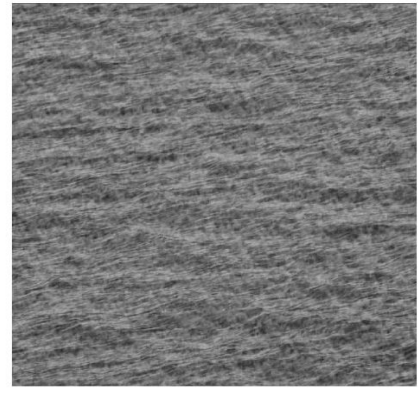

$\mathrm{t}=0.5$

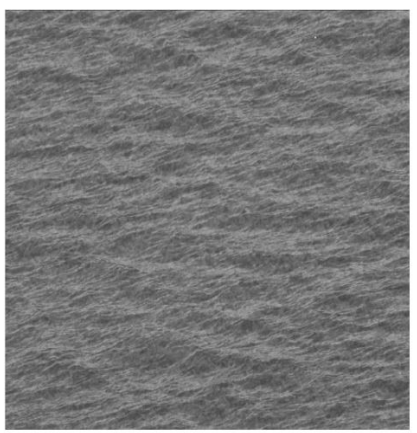

$\mathrm{t}=0.8$

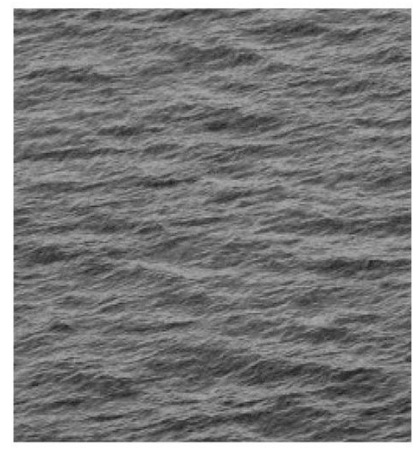

$t=1$

Figure 4.3: Greyscale output keeping the value of $t$ constant throughout the whole output image. 
From Fig. 4.3 we can observe the effect of spatial location on texture distribution in the output. That is why we vary $t$ from 0 to 1 throughout the output plane from left edge to the right edge. Fig. 4.4 shows the greyscale output after combining the levels of $L^{R}$. Here $t$ is changed from left edge to the right edge of the output image plane.

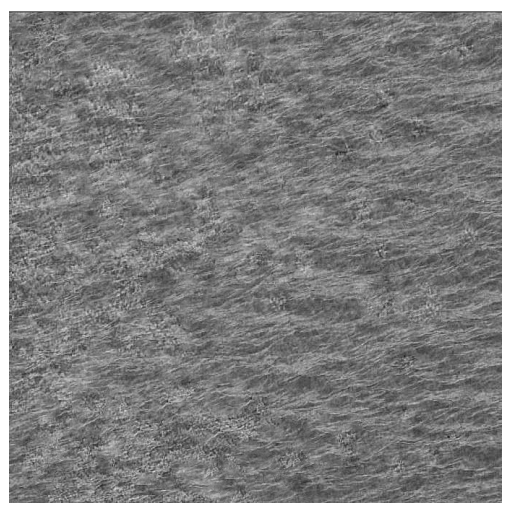

Figure 4.4: Greyscale output combining the levels of resultant pyramid and changing $t$ from 0 to 1 from the left edge to the right edge of output image plane.

In Fig. 4.4, a gradual change in texture contents from grass texture to water textures is observed. The sand texture has more textureness than the water wave texture. We calculate the weights $p_{l}^{\prime}$ and $q_{l}^{\prime}$ in such a way that coefficients from water texture got amplified and preserved in the output.

\subsubsection{Joint SLIC Segmentation}

Previously, we were determining spatial location for each and every coefficient and used that location in the equation for the smooth maximum function. Unfortunately, this led to quantization as adjacent coefficients in the upper levels of the pyramid would have very different blending parameters. The original textures from each input could hardly be recognized in the output. We preferred to bring larger chunks of recognizable textures from each input. In order to achieve this, we applied a SLIC segmentation to create an oversegmentation of the image plane. Within each segment, we use a constant blend factor, and all coefficients contributing to a given pixel use the same blend factor(defined in section 4.1). This 
helps to ensure that the original textures can be recognized individually in the output.

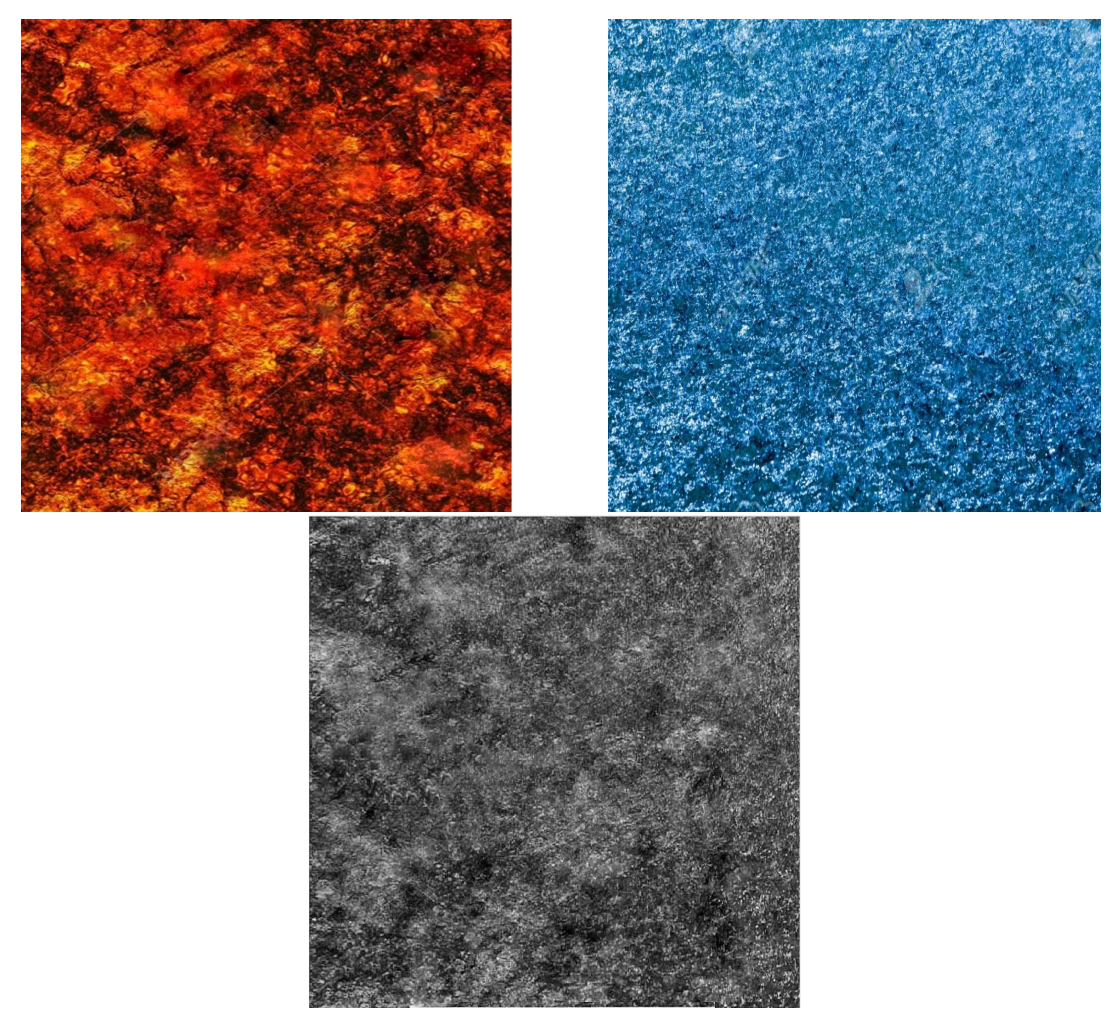

Figure 4.5: Output after the smooth maximum function and reconstruction from Laplacian pyramid where individual texture contents from inputs are not recognizable in the greyscale output.

We explain the reason of using joint SLIC algorithm with the examples in Fig.4.5 and Fig.4.6. The two images in the upper row in Fig. 4.5, are two input textures. The image in the bottom row in Fig. 4.5 shows the greyscale output after combining the levels of resultant Laplacian pyramid, $L^{R}$ without using the SLIC segmentation. In the greyscale output, texture structures from each input can hardly be recognized. The situation can be observed more clearly in a colored example in Fig 4.6. In Fig.4.6, the two images in the upper row are the two input textures of grass and water respectively. The bottom left image in Fig.4.6, shows the colored blended result generated without using the SLIC segmentation. It can be noticed that neither the grass texture nor the water texture can be recognized individually. In the output there is no distinct individual texture from inputs. It is a blend of the grass and the water texture and the combined texture has no real life meaning. 
For preserving the identity of texture contents in the output, we use the SLIC segmentation. The coefficients are blended based on the value of the blend factor at the centroids only. In a SLIC segment, the value of the blend factor, $t$, obtained at the centroid is applied to all the coefficients within that segment. In this way the texture within in this segment can be controlled to be close to the texture of one or the other of the two inputs.
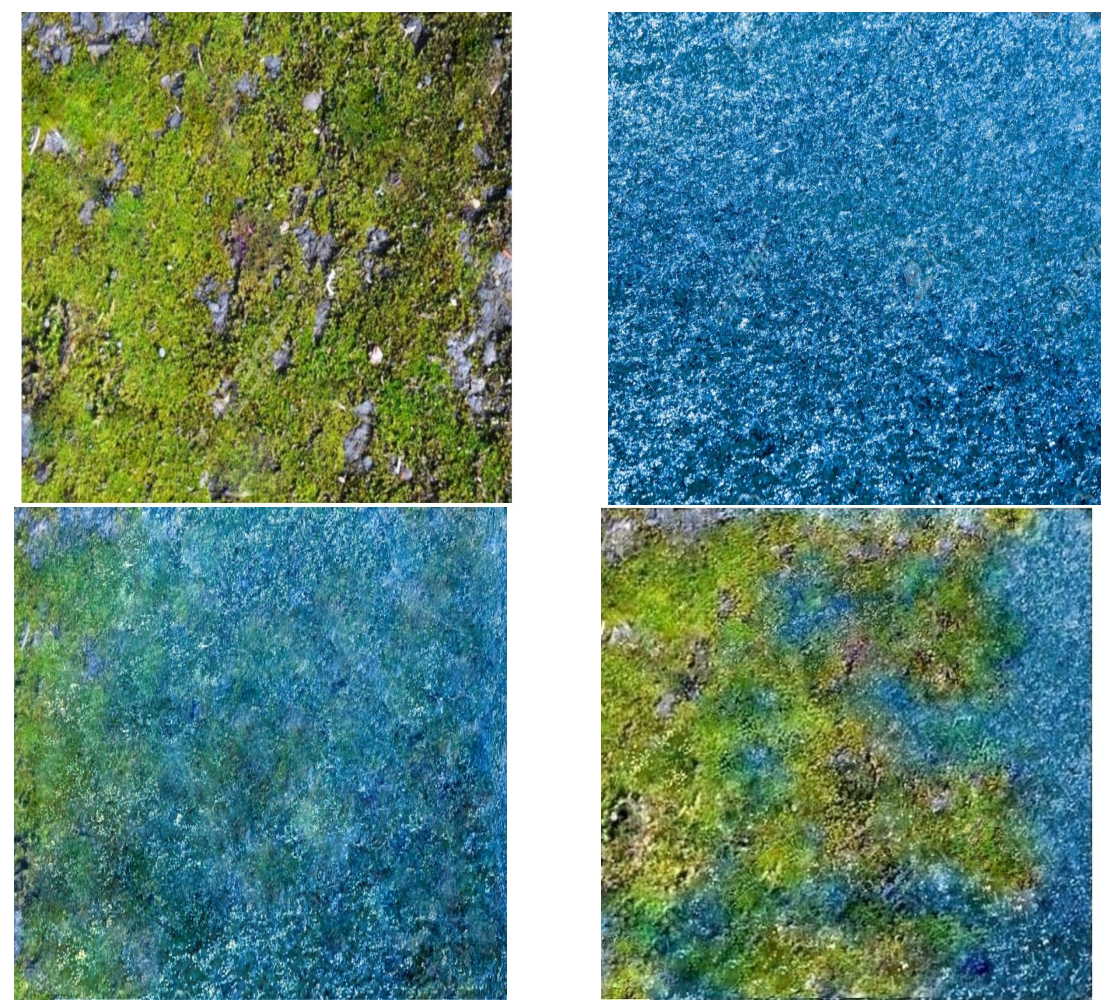

Figure 4.6: Images at the upper row are two inputs. Bottom left is the colored result without using the SLIC segmentation. Bottom right is the colored result after using the SLIC segmentation.

The bottom right image in Fig.4.6 shows the result obtained after using the SLIC segmentation. In this output the water and the grass texture can be identified individually in the output image. We shall describe the SLIC segmentation in our algorithm in detail in our next section. 


\subsubsection{Segmentation With The SLIC Superpixel}

We use the SLIC segmentation in order to distinguish the textures or to preserve the actual textures from both inputs after blending. The coefficients are blended inside the SLIC segments. In this section we are describing this step.

We create a segmentation map for the output. Each segment will have a particular mix of texture from the output. We want the segment shapes to conform to details from the input textures, so we apply a segmentation that considers both inputs simultaneously.

In the original SLIC formula, the spatial distance and the color distance are measured in a single image. In our modified SLIC segmentation, we use two color distances: the distance of the centroid from the colors of the pixels in both inputs. The spatial distance remains unchanged.

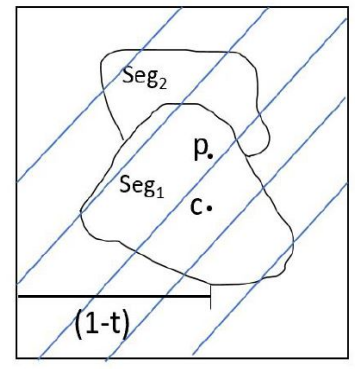

(a) Input 1 ( $\left.A^{\prime}\right)$

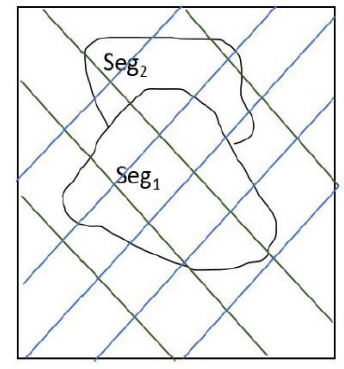

(b) Output

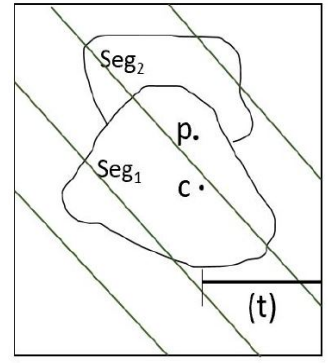

(c) Input $2\left(B^{\prime}\right)$

Figure 4.7: Sample image explaining how the SLIC segmentation works in our experiment. SLIC segmentation is done in any one of the inputs but in the segmentation formula, color distances of pixels at same location in both inputs, from their corresponding centroid location are considered. Color distances are measured with Eqn. 4.8 and Eqn. 4.9.

Fig. 4.7 is a sample image for describing the procedure. Let Fig. 4.7 (a),(c) represents the inputs and Fig. 4.7 (b) represents the output. We have done the joint SLIC segmentation, where the color distances of pixels at same location in both $A^{\prime}$ and $B^{\prime}$ from their corresponding centroid pixels are used. The contributions of both color distances to the total distance are weighted by the blend factor: the segments 
will be governed by input A when $t$ is near zero, and by input $B$ when $t$ is near unity.

Suppose that in a segment $S e g_{1}$, we have a centroid located at $c$. Here, $d_{r g b}^{A^{\prime}}$ is the color distance of a pixel in $A^{\prime}$ from its centroid and $d_{r g b}^{B^{\prime}}$ is the color distance of a pixel in $B^{\prime}$ from its centroid. Let we find $d_{r g b}^{A \prime}$ for the pixel at location $\mathrm{p}$ in $A^{\prime}$ from Eqn. 4.8. Then we find $d_{r g b}^{B \prime}$ for the pixel at the same location $\mathrm{p}$ in $B^{\prime}$ from Eqn. 4.9. We calculate the color distances for all the pixels. Then we use both $d_{r g b}^{A \prime}$ and $d_{r g b}^{B \prime}$ in the SLIC segmentation formula. By iterating as usual, we compute the full joint SLIC segmentation.

In summary, the joint distance formula is as follows:

$$
\begin{gathered}
d_{r g b}^{A^{\prime}}=\sqrt{\left(\left(r_{p}^{A^{\prime}}-r_{c}^{A^{\prime}}\right)^{2}+\left(g_{p}^{A^{\prime}}-g_{c}^{A^{\prime}}\right)^{2}+\left(b_{p}^{A^{\prime}}-b_{c}^{A^{\prime}}\right)^{2}\right)} \\
d_{r g b}^{B^{\prime}}=\sqrt{\left(\left(r_{p}^{B^{\prime}}-r_{c}^{B^{\prime}}\right)^{2}+\left(g_{p}^{B^{\prime}}-g_{c}^{B^{\prime}}\right)^{2}+\left(b_{p}^{B^{\prime}}-b_{c}^{B^{\prime}}\right)^{2}\right)} \\
D_{s}=(1-t) \cdot d_{r g b}^{A^{\prime}}+t \cdot d_{r g b}^{B^{\prime}}+\frac{m}{S} \cdot d_{(x, y)}
\end{gathered}
$$

where

$$
S=\sqrt{\frac{N}{K}}
$$

as before.

The spatial distance $d_{(x, y)}$ is the $\mathrm{x}-\mathrm{y}$ plane distance of the centroid normalized by the grid interval S. In Eqn. 4.11, $N$ is the number of pixels in the image and $K$ is the number of segments. For every pair of pixels in $A^{\prime}$ and $B^{\prime}$, their color distances and spatial distances from their corresponding centroids are taken into consideration. The parameter $m$ governs the compactness of the superpixel. The greater the value of $m$, the more spatial proximity is emphasized and the more compact the cluster. We used $m=10$ for all of the results in this experiment. We introduced the value of $t$ in the SLIC equation so that the value of $t$ has an effect on the overall distance $D_{s}$. 
We create a bounding square for each of the SLIC segment in order to create the Laplacian pyramid for each segment. We generate the Laplacian pyramid for the bounding square and coefficients are blended at each level of the pyramid. Thus we followed the steps of sub-section 4.2.1 for the coefficient blending. For each segment, at each level of the Laplacian pyramid, we find the value of $t$ at the centroid only. Then we apply the same value of $t$ in the smooth maximum function for all the coefficients within that segment.

All the coefficients inside a SLIC segment have the same value of $t$ as the weighting factor. That is why, most of the output coefficients after blending are either close to the input coefficient, $u$ from $A^{\prime}$ or input coefficient, $v$ from $B^{\prime}$. This maximum number of inclination towards any one of the input coefficients helps in preserving the actual texture structure in the output. That is why the distinguishable texture features from each inputs are retained in the output even after blending.

Once the coefficients are blended, we keep only the blended coefficients within the original SLIC segment boundary from the bounded square. We discard the rest since the bounded square was created only for generating the Laplacian pyramid. The coefficients that were not discarded form a temporary pyramid that can be collapsed to produce pixel values within the segment. Once the pixel values within the segment have been computed, we discard the temporary pyramid. This is done for all $K$ of the SLIC segments. This process is akin to that taken by Paris et al. [32]. The number of segments, $K$ affects the size of the segments. The size of the segments has a visual effect on the output blended textures.

\section{Random Location Selection}

In the output image we aim for having a texture transition from left to right by keeping texture contents of the left input towards left and texture contents of the right input towards the right region of output. At the same time we also want a bit of scattered distribution of texture contents from both input. Irregular shape of the SLIC segments helps in getting an irregular, realistic result. To achieve a bit more irregular distribution of texture, we take one additional simple step of random location selection. 
The value of $t$ increases from 0 to 1 as t moves from left edge to right edge of resultant image. Ideally we want resultant image to be like input texture, $A^{\prime}$ when $t \approx 0$ and like input texture, $B^{\prime}$ when $t \approx 1$. But we are taking this step so that when $t \approx 0$ there is some textures from $B^{\prime}$. When $t \approx 1$ there is some textures from $A^{\prime}$. This is done for $10 \%$ of the number of SLIC segments.

We chose some random number, $r$ within the range of 0 and 1 . The value of $p^{\prime}$ and $q^{\prime}$ can be controlled in Eqn. 4.4 and 4.5 by altering value of $t$. In Eqn. 4.4 and Eqn. 4.5, $p$ and $q$ is multiplied with $(1-t)$ and $t$ respectively. Now, at a given location of centroid , $t$, if $r>t$, then Eqn. 4.5, Eqn. 4.6 is modified as,

$$
\begin{aligned}
& p^{\prime}=p \cdot \max (t, 1-t) \\
& q^{\prime}=q \cdot \min (t, 1-t)
\end{aligned}
$$

if $r<t$, then Eqn. 4.5, Eqn. 4.6 is modified as,

$$
\begin{aligned}
& q^{\prime}=q \cdot \max (t, 1-t) \\
& p^{\prime}=p \cdot \min (t, 1-t)
\end{aligned}
$$

Through this additional step we can retain some texture contents from right input towards the left region of output. Within the right region of output we can bring some texture contents from the left input. This gives a scattered and irregular distribution of texture contents from both inputs throughout the output image.

Fig. 4.8 shows the region map with SLIC segments where it is observed how texture contents of both the inputs are distributed. The regions with grey marking are populated with the right image contents and the white regions are populated with the left image contents. Contents from both inputs are evenly distributed except at the left most and the right most region. At a certain location, if the output coefficient is closer in value to the left input coefficient in more Laplacian pyramid levels than that of the right image, we assign white color to that location in the map. Otherwise, we assign grey color in the map. 
Within a SLIC segment, majority of output coefficients are close to either left input coefficients or the right input coefficients. This happens due to the biasing of our calculated weights in smooth maximum function. Even after biasing, some output coefficients do not follow the majority of the output coefficients within a segment. That is why, we can see some white dots in the grey segments and some grey dots in the white segments in Fig. 4.8.

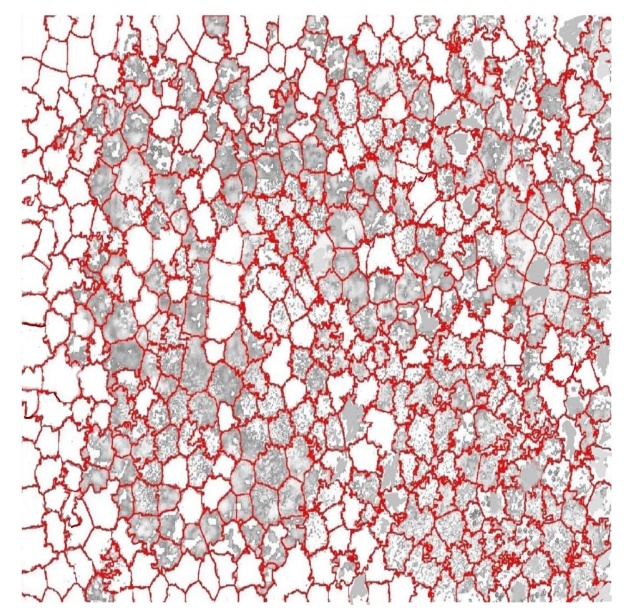

Figure 4.8: The SLIC segments map. Here the regions with grey marking are populated with right image contents and white regions are populated with left image contents. Contents from from both inputs are evenly distributed except at the left most and right most corner.

Due to the scattered and irregular distribution, the merging of two texture inputs give a gradual flow without any sudden change at any point. The left most region is totally input $A^{\prime}$ and right most region is totally input $B^{\prime}$. Within the middle area there is an uneven distribution of textures from both inputs.

Combining the levels of resultant pyramid we get a greyscale blended segment. If there are K number of segments in each input then we get K blended segments. All the blended segments together constitute the output greyscale image.

\subsubsection{Assigning Color And Smooth Merging At Halo}

At this step of the algorithm we assign colors to the greyscale image. The greyscale image is obtained after merging the levels of the resultant Laplacian pyramid. After 
assigning colors, at some regions there are some discontinuities or sudden changes of color appear in the output. This occurs where texture contents with totally different colors are lying side by side along the boundaries of the SLIC regions. We name these regions as halo regions. We do the alpha blending of colors along the halo regions.

After combining all the levels of the resultant Laplacian pyramid, the greyscale result is obtained. For getting the RGB image we use the precomputed look-up tables which are storing the pixel values of the inputs. Each input has their own precomputed look-up table. We mentioned about generating this look-up table in Chapter 3.

Therefore each input image $A$ and $B$ has their own precomputed lookup table. To assign color to the greyscale image, we have to decide from which lookup table the RGB values will be chosen. At each level of the Laplacian pyramid, coefficient mixing was done. The output of the coefficient mixing was either close to the value of coefficient of $A^{\prime}, u$ or close to the value of the coefficient of $B^{\prime}, v$. At one level of the pyramid, the output coefficient might be close to $u$ and at another level it might be close to $v$. Color from which look-up table to choose from, was decided by the maximum number of times the output coefficient is close to either $u$ or $v$ in all the levels of the resultant pyramid.
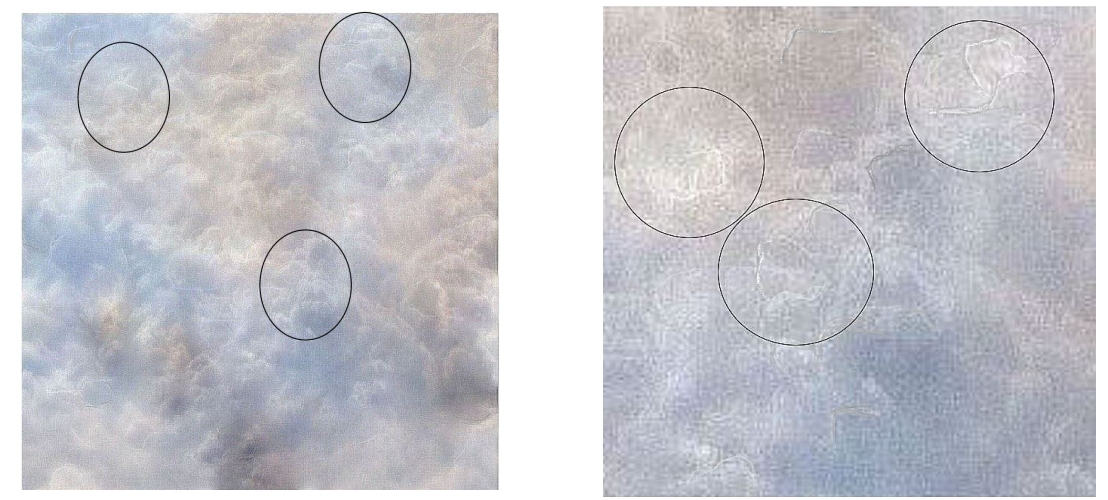

Figure 4.9: SLIC segments boundary edges are noticed subtly (shown inside circles) if two texture elements across the boundary are very much dissimilar in color and contents 


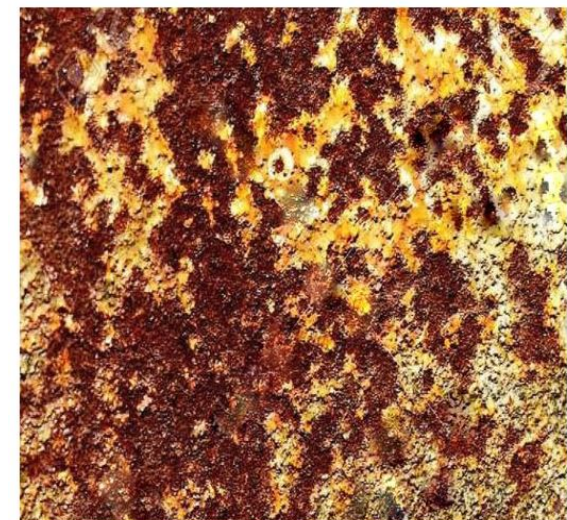

(a)Input1

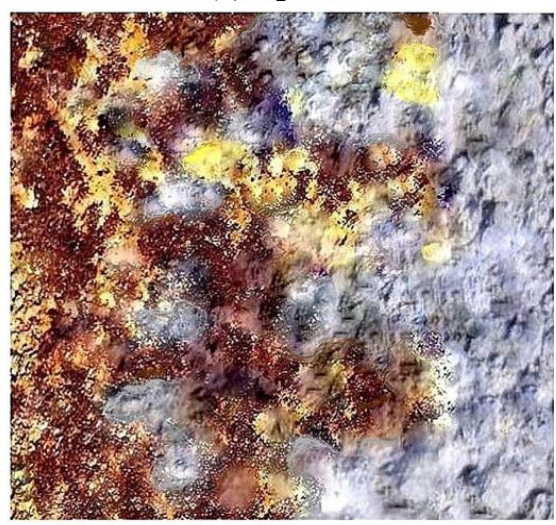

(c) Output after blending without smooth merging at Halo

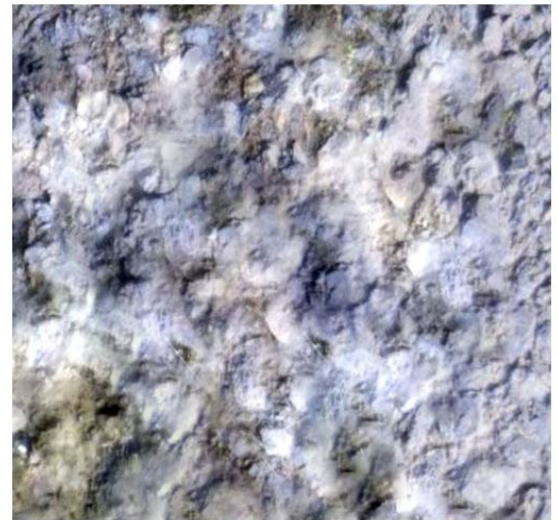

(b)Input 2

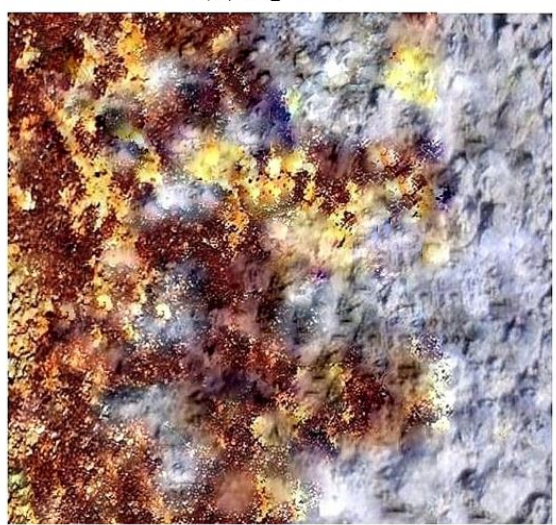

(d) Output after blending after smooth merging at Halo

Figure 4.10: Some SLIC segments are very subtly visible (in Fig. (c))if two different colors are present on each side of the SLIC segments.

After populating the segments with texture contents from both inputs and after assigning colors, there are sharp edges and discontinuities noticed at the boundary of some SLIC segment. This happens if two texture contents from two inputs lie side by side and at the two sides of the boundary there is large change in colors. We describe the phenomenon with the help of two examples shown in Fig. 4.9, Fig.4.10 and Fig.4.11. In the Fig. 4.9 and Fig.4.11 we can notice sharp edges along the boundaries where different colors are present in both sides of the boundaries. 


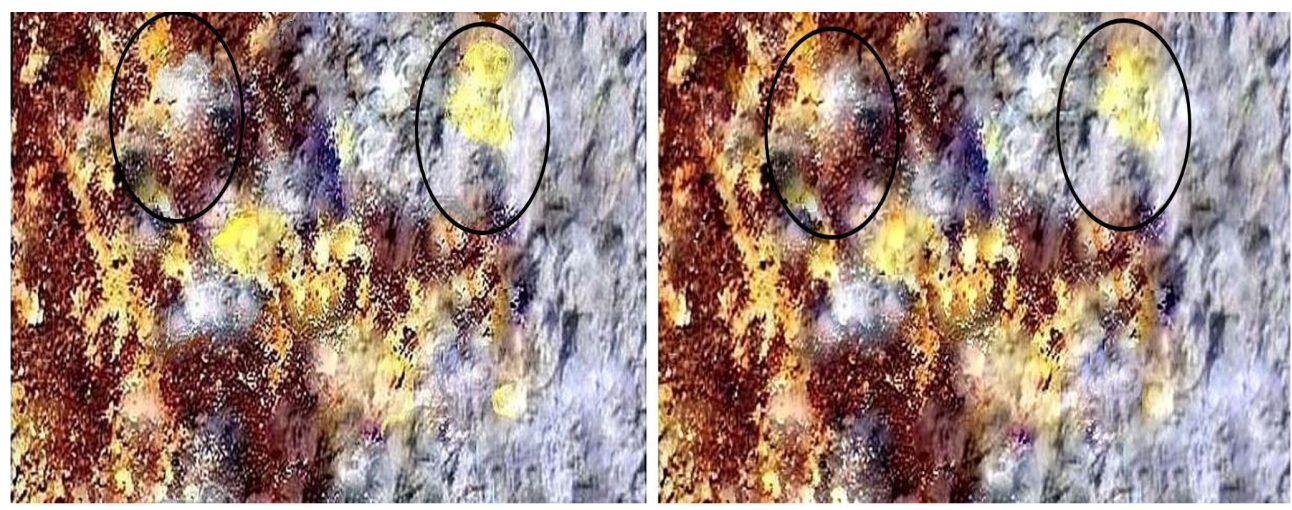

Figure 4.11: Boundary edges are subtly visible before the alpha blending (left image). After the alpha blending of colors along the edges of the SLIC segments, the boundary edges of segments are not visible (right image).

Let the textures from $A^{\prime}$ and $B^{\prime}$ lying side by side on either side of a SLIC boundary. If the colors of those textures are very different then a sharp change of color becomes noticeable along that SLIC boundary. That is why, along the pixels of the boundary we did alpha blending of two colors lying on either side of the boundary. It makes a gradual change in color along that boundary pixels. For example in Fig.4.12, if the boundary pixels are on $\left(x_{i-n}, y_{i-n}\right),\left(x_{i-(n-1)}, y_{i-(n-1)}\right), \ldots,\left(x_{i}, y_{i}\right),\left(x_{i+1}, y_{i+1}\right), \ldots,\left(x_{i+n}, y_{i+n}\right)$, then along these pixels we do the alpha blending of colors. From $-n$ to $+n$ we vary $\alpha$ from 0 to 1.

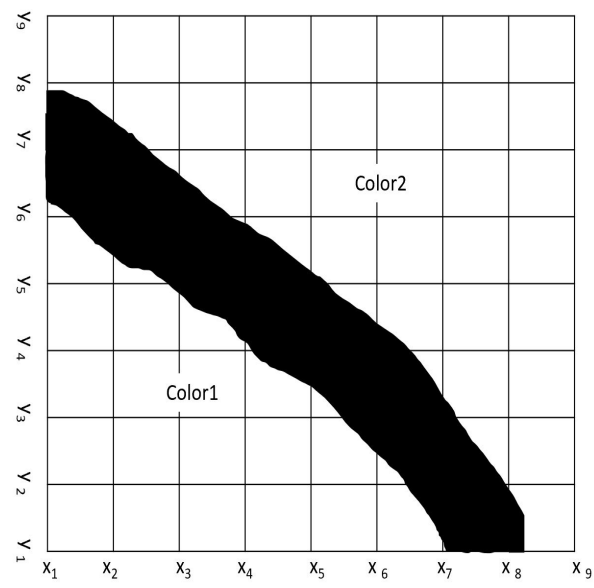

Figure 4.12: This is a zoomed representation of a SLIC boundary. Along the boundary pixels, $\left(x_{i-n}, y_{i-n}\right), . .,\left(x_{i}, y_{i}\right), . .\left(x_{i+n}, y_{i+n}\right)$, alpha blending of colors is done. 
This contributes in making a smooth transition between the different input contents. This boundary pixels are located throughout several rows and columns of image space and we term these locations as the halos. 


\section{Chapter 5}

\section{Results and Discussion}

\subsection{Outcome With Different Textures And Evalu- ation}

In the previous chapter we described the steps of our algorithm for generating the images with texture transition. In this chapter we are showing the results for various input textures. Then we shall describe the results and mention the time taken for our algorithms. Then we shall describe the failure cases.

We generated some random phase textures with no definite shapes and orientations using our algorithm. One of the weighting factors in our algorithm is $t$ which denotes the proportion of the texture elements from image input $A$ and input $B$. We used $t$ in our algorithm as measure of spatial distance along the $\mathrm{x}$-axis of the image plane. Therefore, the presence of texture contents from input A and input B depend on the spatial location of the textures along the horizontal dimension of the image plane.

Another factor controlling the weight of the texture contents from input A and input $\mathrm{B}$ is textureness of the inputs. In the smooth maximum function, we calculate the weights in such a way that the input with lower textureness is amplified. Otherwise the textures with lower textureness would always be lost in our coefficient mixing process.

We use the SLIC segmentation in our algorithm and the size of the segments also affects the output. We can notice the texture contents from inputs appearing in 
chunks of different sizes depending on the size of the SLIC segments. Different sizes of the SLIC segments create a visual impact on the outputs.

We used some of the outputs generated by the histogram preserving blending as the inputs of our algorithm. We also used some inputs which are not random phase inputs. Thus we used those non-random phase inputs directly without using the histogram preserving blending algorithm. In the following sub-section, first we show all the results with different types of inputs. Then in the next sub-sections we show how different parameters affect the results. We show some results, where the inputs are of similar color textures. We also show the results where the inputs are of totally different textures and colors.

\subsubsection{Results With Different Types Of Inputs}

The inputs of results in Fig. 5.1 are the outputs of the histogram preserving algorithm [22]. The images at the right and left column are the inputs. The images at the middle column are the blended outputs of the two inputs. In the topmost row, the inputs are very much similar both in color and texture contents. Still the transition from one texture to another texture can be noticed from the left edge to the right edge of the output. The middle row shows a blend between two textures dissimilar both in structure and in color. The oversegmentation with the SLIC produces islands of one color or the other. Although the colors do not blend neatly here, the result admits a semantic interpretation of water sinking into sand. This result shows the limitation of our color blending process in the oversegmentation context and points the way towards future work. From the result in the third row we can observe the dominance of the cloud texture in the output. This is because of the higher textureness of the cloud texture compared to the sky image.

The inputs on the left and the right column in Fig. 5.2 are some images with non-random phase textures. The results at the middle column show the blended outputs with inputs having well-defined structures. It can be observed in all of the results that the regular structures of the inputs are distorted.

In Fig. 5.3, the inputs are used directly without using the histogram preserving 
algorithm. The inputs are on the left and the right column and the inputs do not have any well-defined structures. That is why the textures are smoothly merged. The result in the third row shows the semantic interpretation of some stone patches across the grass field.

Similarly in Fig. 5.4, the inputs are taken directly without using the histogram preserving blending. The inputs are on the left and the right column and the images at the middle are the blended outputs. In both of the upper and the bottom row, there are no similar texture or color contents in the inputs. Even there is no semantic interpretation of the merged output texture. This figure shows an example of merging any two arbitrary input textures with no similarity of color and texture contents and having no semantic interpretation. 

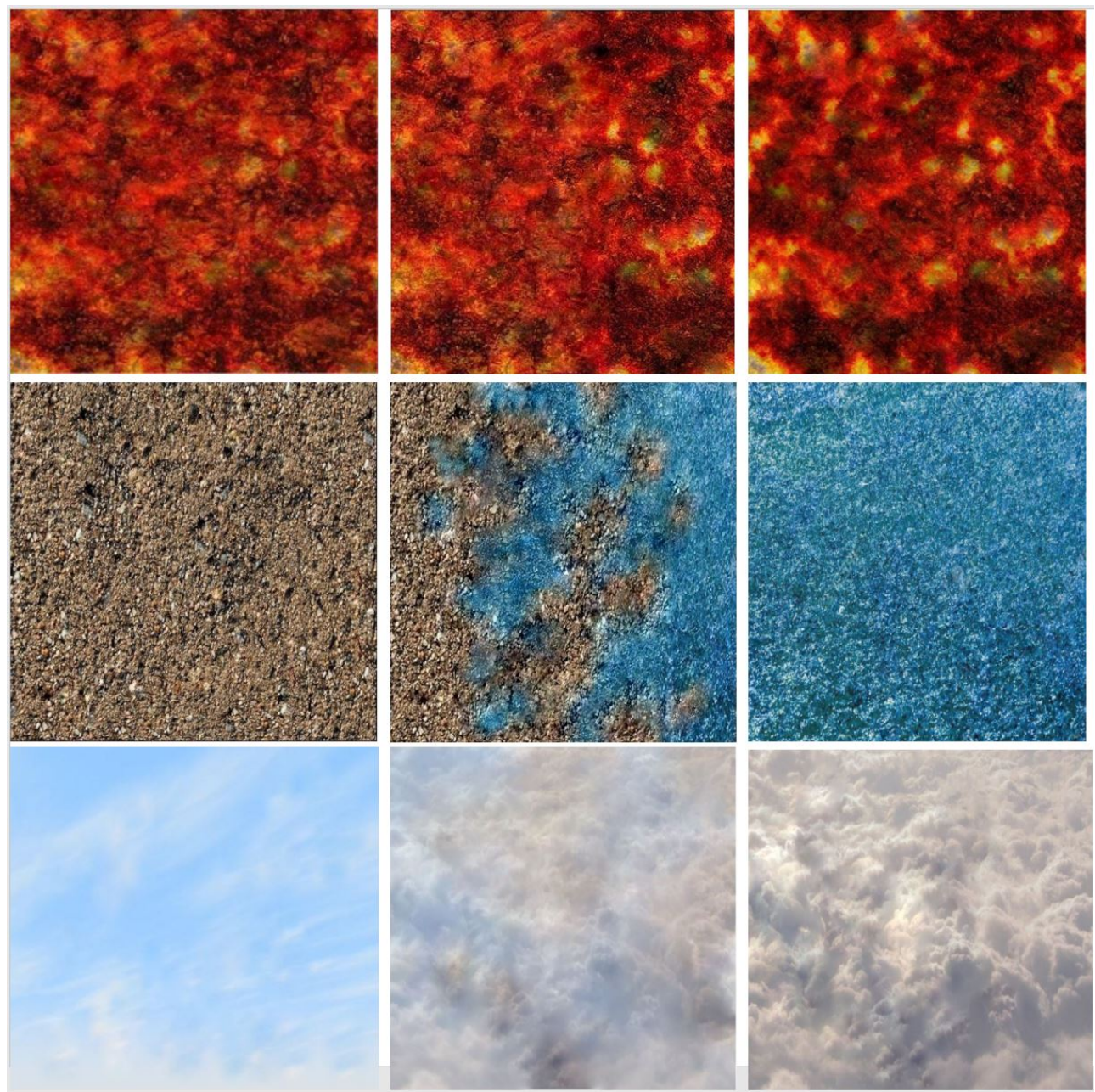

Figure 5.1: Results at the middle column showing the blended image of two inputs at left and right. The inputs are generated by the histogram preserving blending algorithm [22] 

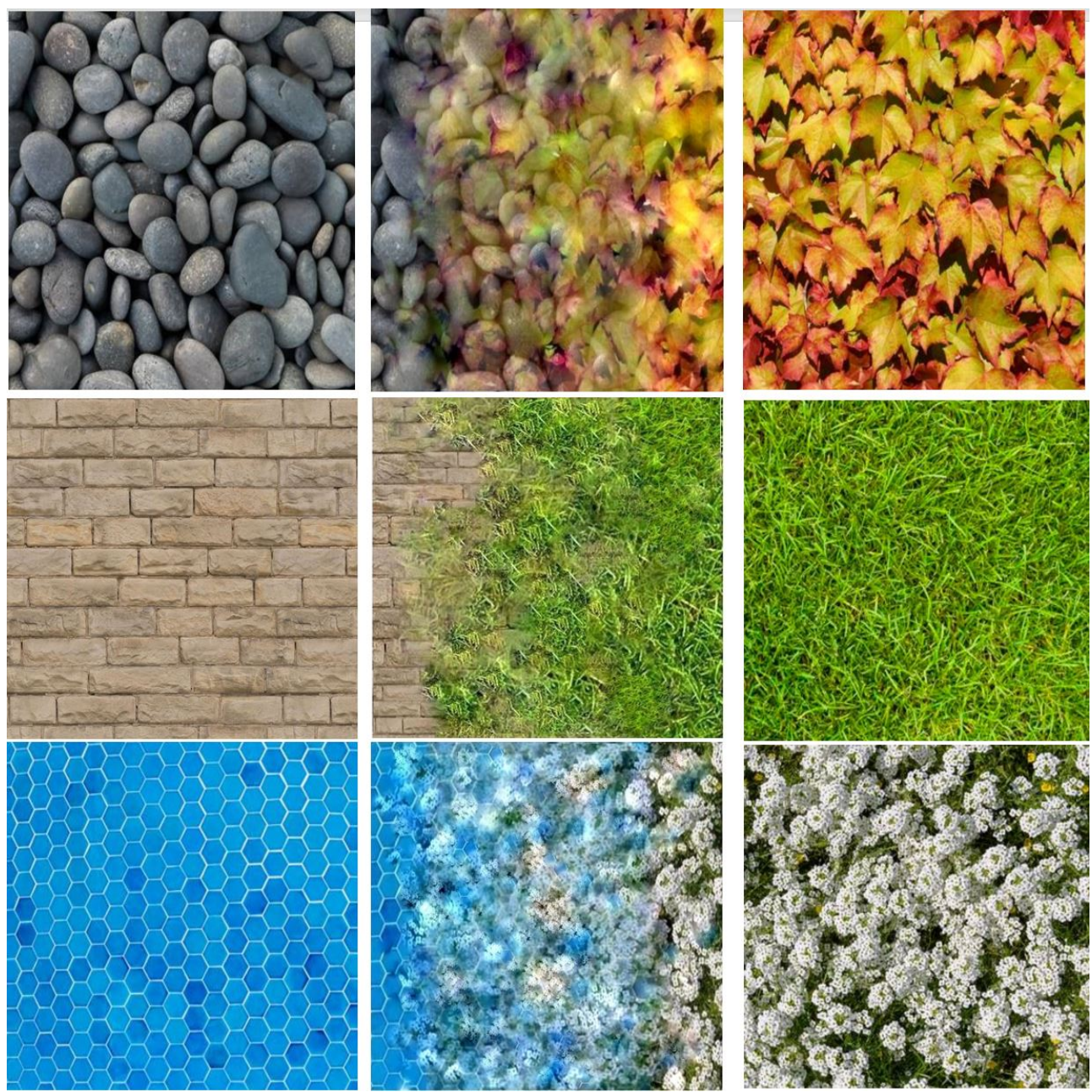

Figure 5.2: Results at middle column showing blending of non-random phase textures(left and right column are inputs [25], [24], [41], [15]). The inputs are used directly without using histogram preserving algorithm 

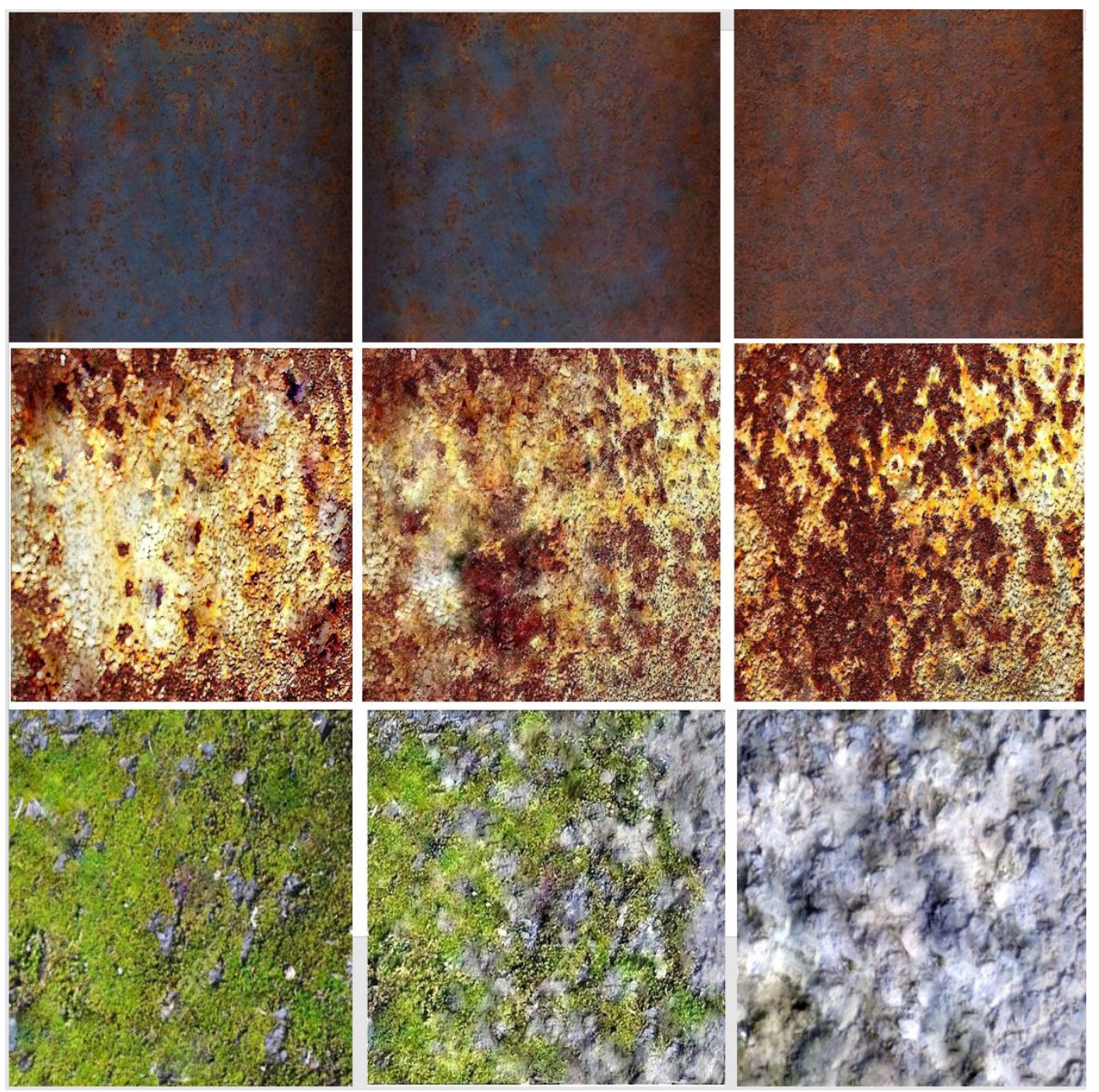

Figure 5.3: Results at middle column showing blending of non-random phase textures(left and right column are inputs). The inputs are used directly without using histogram preserving algorithm 

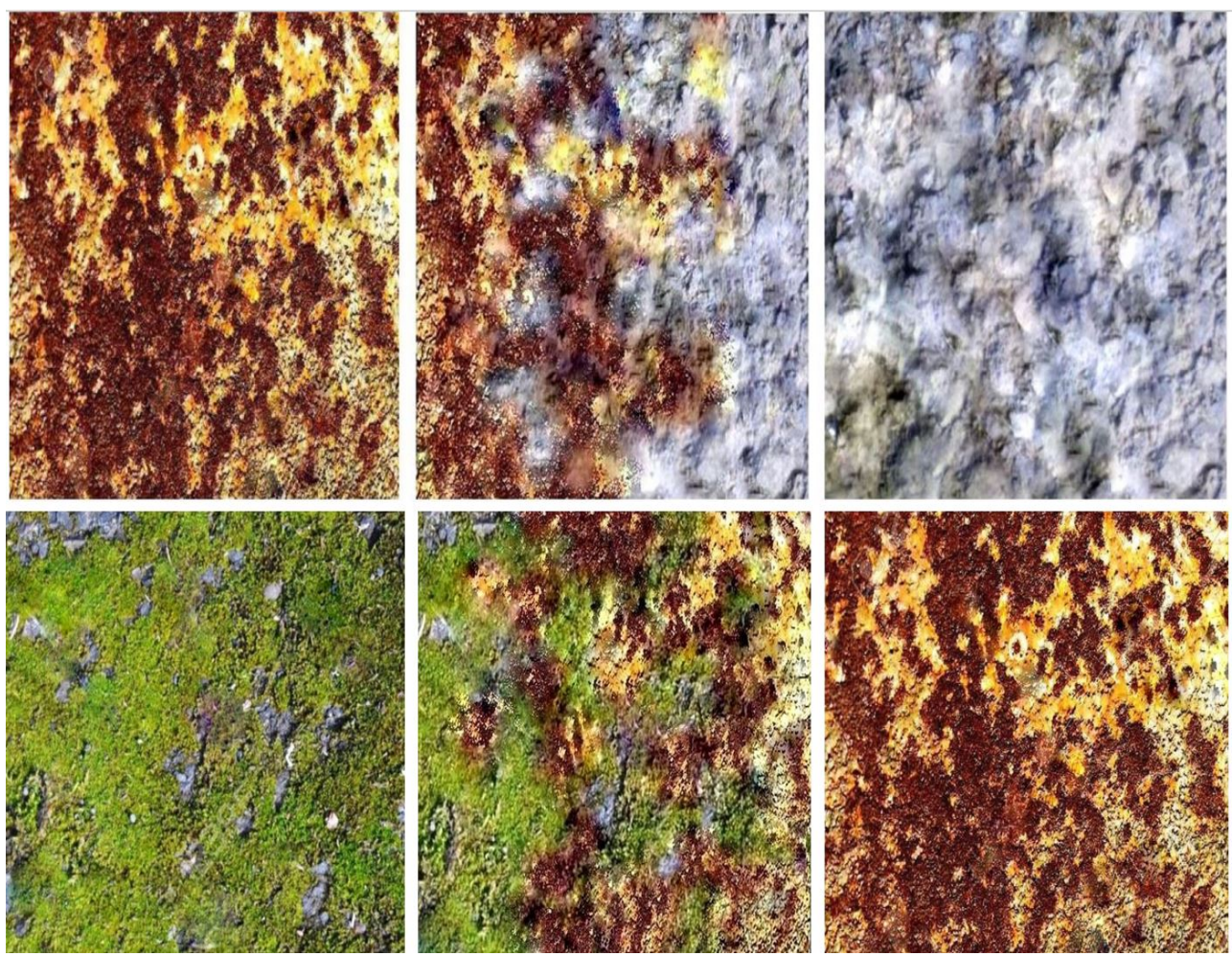

Figure 5.4: Results at middle column showing blending of non-random phase textures(left and right column are inputs).The inputs are used directly without using the histogram preserving algorithm 

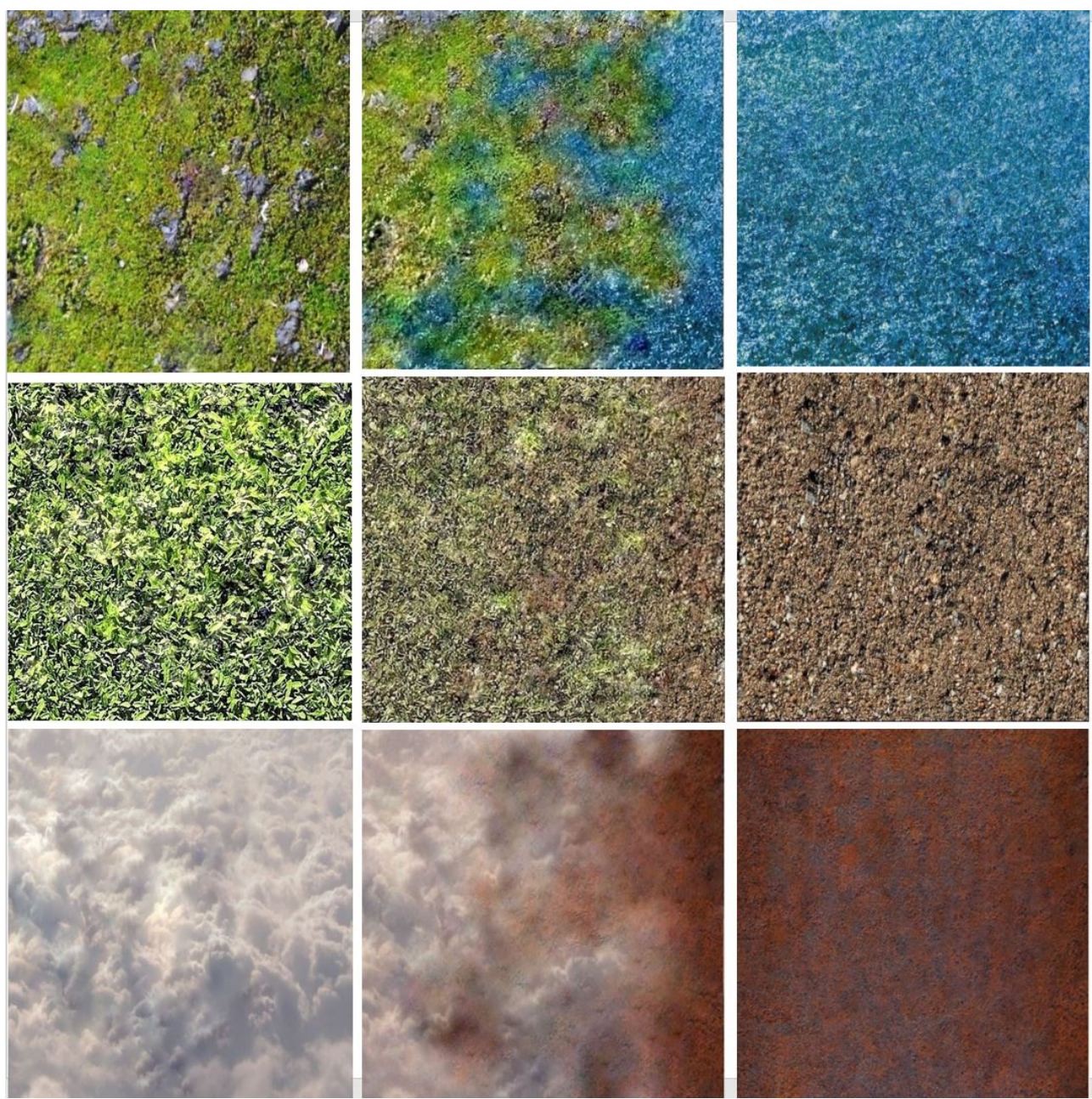

Figure 5.5: Results of our algorithm at the middle column with both direct input and input generated by histogram preserving algorithm(inputs are at left and right column) 


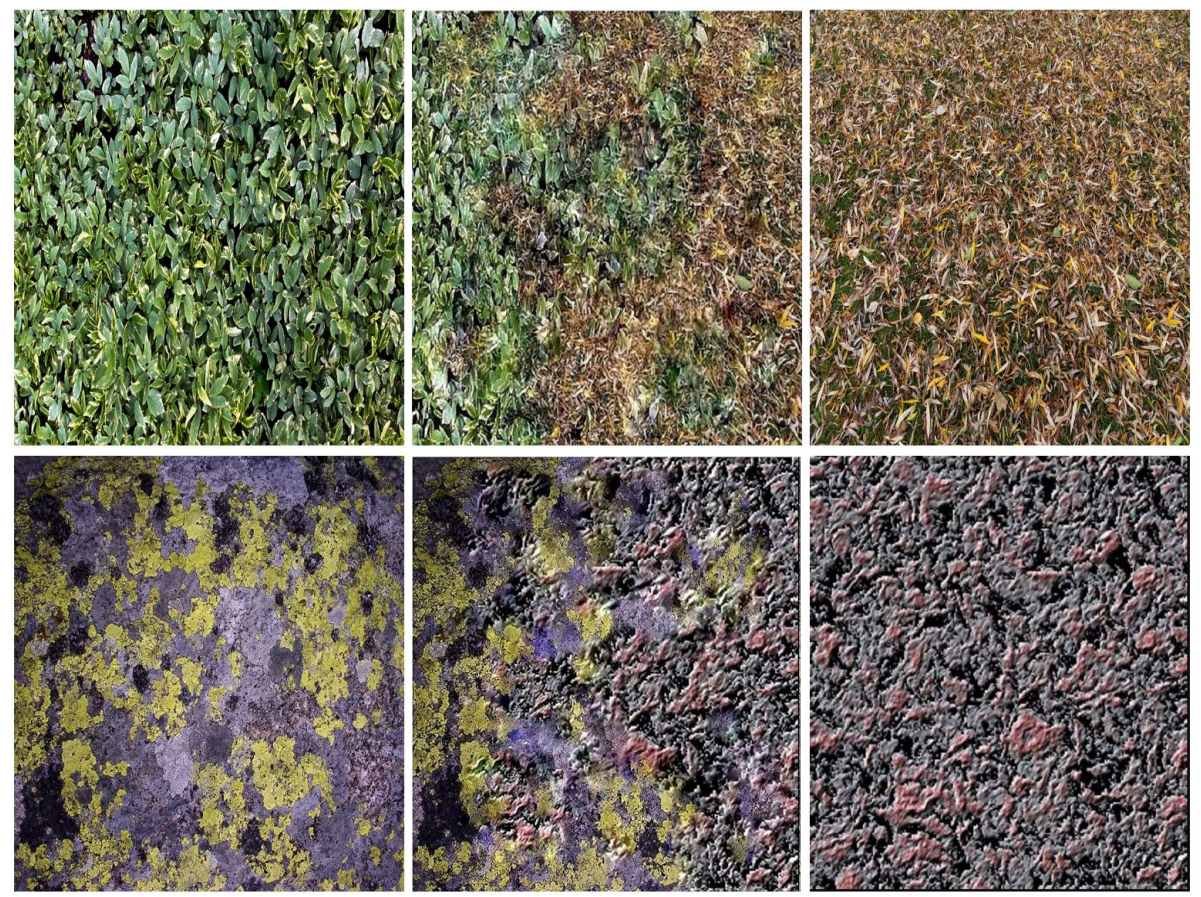

Figure 5.6: Results at middle column showing blending of arbitrary inputs(left and right column are inputs). The inputs [37], [6], [36], [20] are used directly without using the histogram preserving algorithm

In Fig. 5.5, the inputs are on the left and right column and the outputs are on the middle column. Here we have shown examples where we have taken one input directly and the other input is the output of the histogram preserving blending algorithm [22]. We re-implemented the histogram preserving blending and used some of the outputs as the inputs of our algorithm. All the results in the middle column shows the gradual seamless transition of textures from the left input texture contents to the right input contents .

Fig. 5.6 shows two results from blending inputs directly, without the step of histogram-preserving blending to create new texture. In the top row, we see a mix of small leaves of different colors The result scatters small patches of green leaves across the brown leaves. In the bottom row, we see a complex stone face merge with lichens. Again, the structural preservation is good, with the lichens seeming to conform to the shapes in the stone. In both examples, we use oversegmentation, with a blend factor favoring the left input on the left side of the output, and favoring the right 
input towards the right.

\subsubsection{Results without using the SLIC segmentation}

In this sub-section we are showing some results which are produced without using the SLIC segmentation. Here, we did not use oversegmentation with SLIc but simply blended coefficients with the blend factor changing linearly from left to right. If the users do not want the original patches of texture elements from the inputs, then they can omit the step of oversegmentation with the SLIC.

The results in Fig. 5.7 are generated without using the SLIC segmentation. The input textures in both of the results at the middle column merge seamlessly, with features from each integrating nicely. There is no ghosting as would have been produced by alpha blending. 

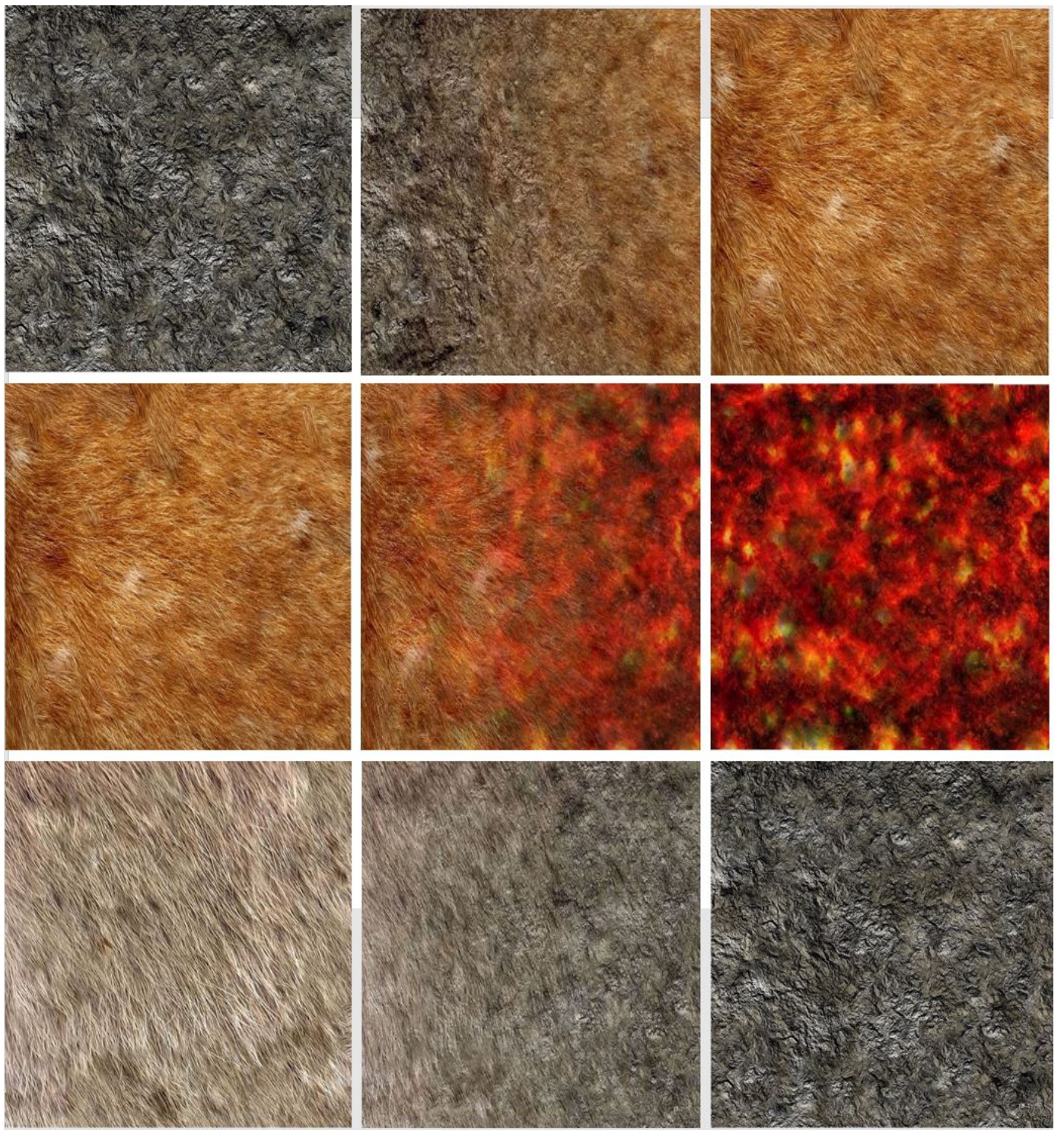

Figure 5.7: Results at the middle column are generated without using the SLIC segmentation. The inputs at the left and the right column are generated using the histogram-preserving algorithm.

\subsubsection{Results With Progressively Variant Texture Contents}

In every results in Fig. 5.8 we can see there is a gradual texture transition from left edge to the right edge of the output image. In our experiments we have varied $t$ horizontally along the x-axis of the image plane. Therefore, in all the results of our experiment, the proportion of texture contents from inputs depend on the spatial location of the texture contents. 
Along with the gradual transition there is also some randomness in the texture distribution throughout the whole output image. This gives a realistic appearance. At several regions of every output, the exact texture contents from inputs are not preserved. The coefficients of pixels in the Laplacian pyramid are changed after the smooth maximum function and even after normalization the actual values cannot be retained. Therefore there is some loss of exact texture elements in every results.
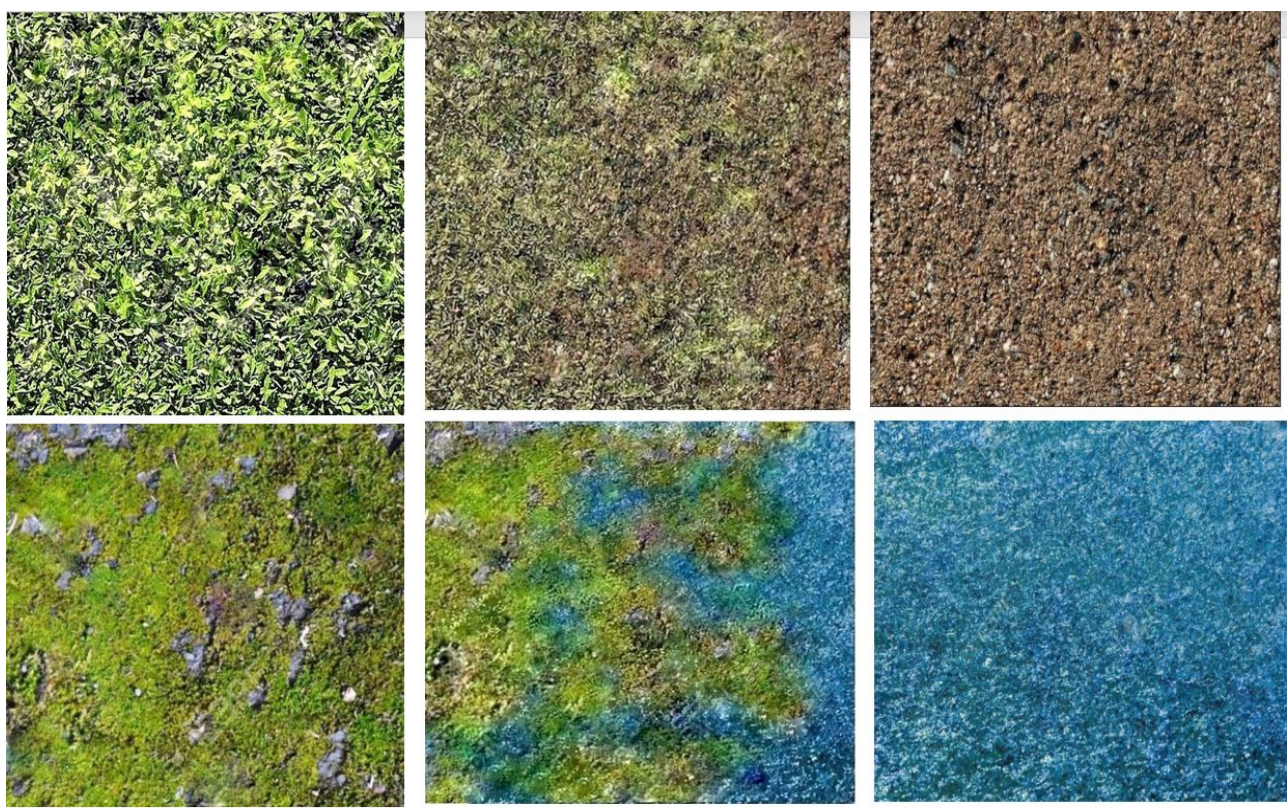

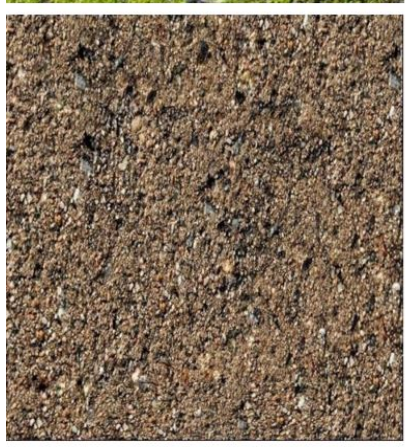

Input A

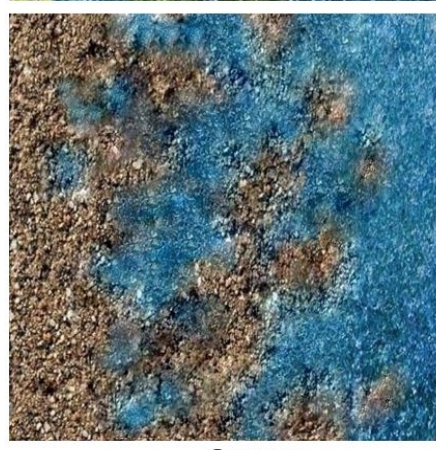

Output

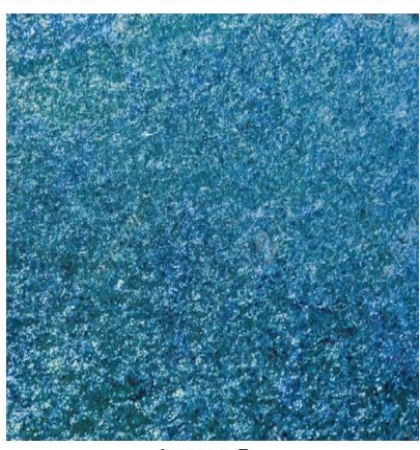

Input B

Figure 5.8: Results at middle column showing progressively variant textures from left to right edge of output image plane. 


\subsubsection{Results Of Blending Two Textures With Different Tex- tureness}

As we have defined before that, by high textureness we mean the local high frequency contents in the texture. In our algorithm we formulated the weights in the smooth maximum function in such a way that the textures with lower textureness in the inputs get amplified and preserved in the output. We amplify the coefficients having lower rms value while coefficient mixing .
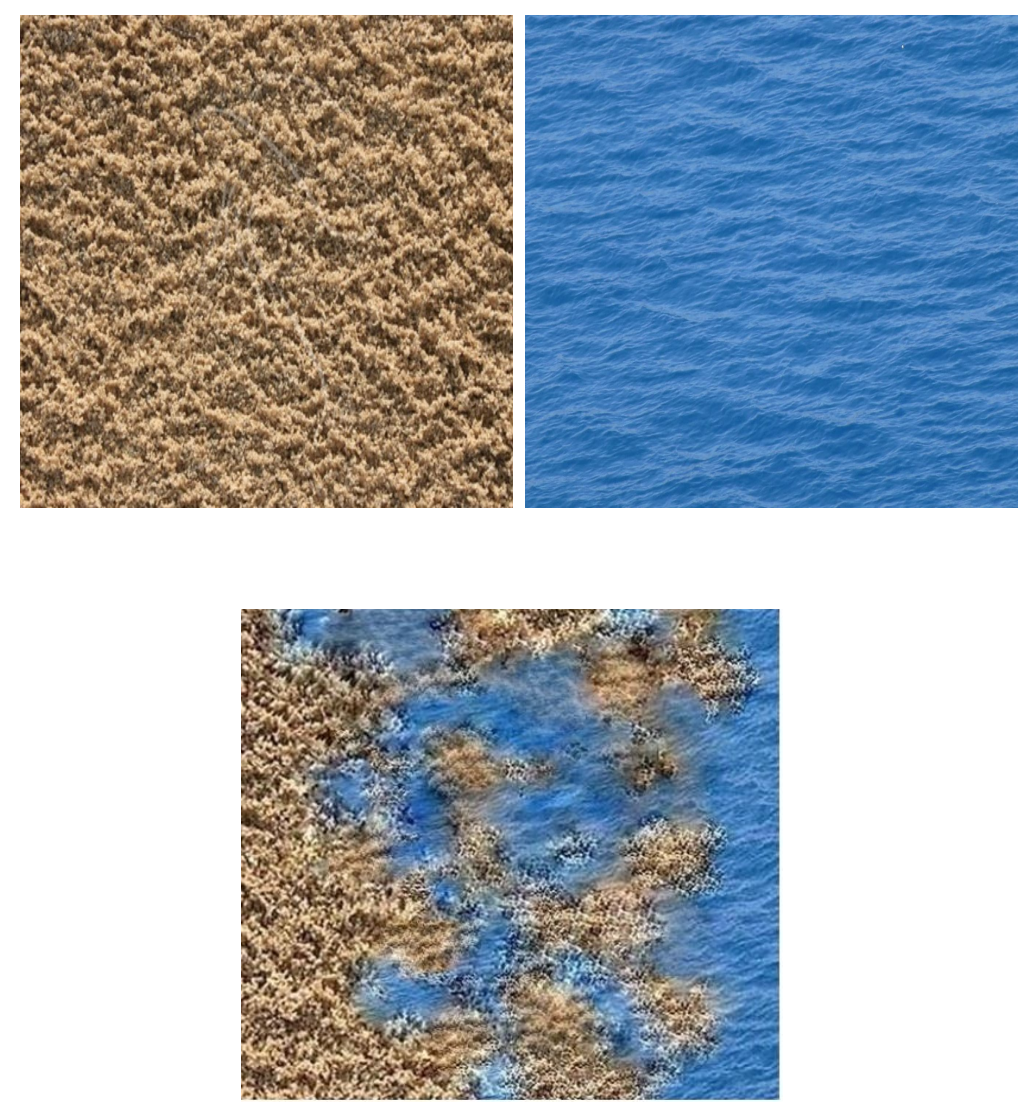

Figure 5.9: Result of blending two textures with different textureness. The images in the upper row are inputs. The bottom image is the output. 

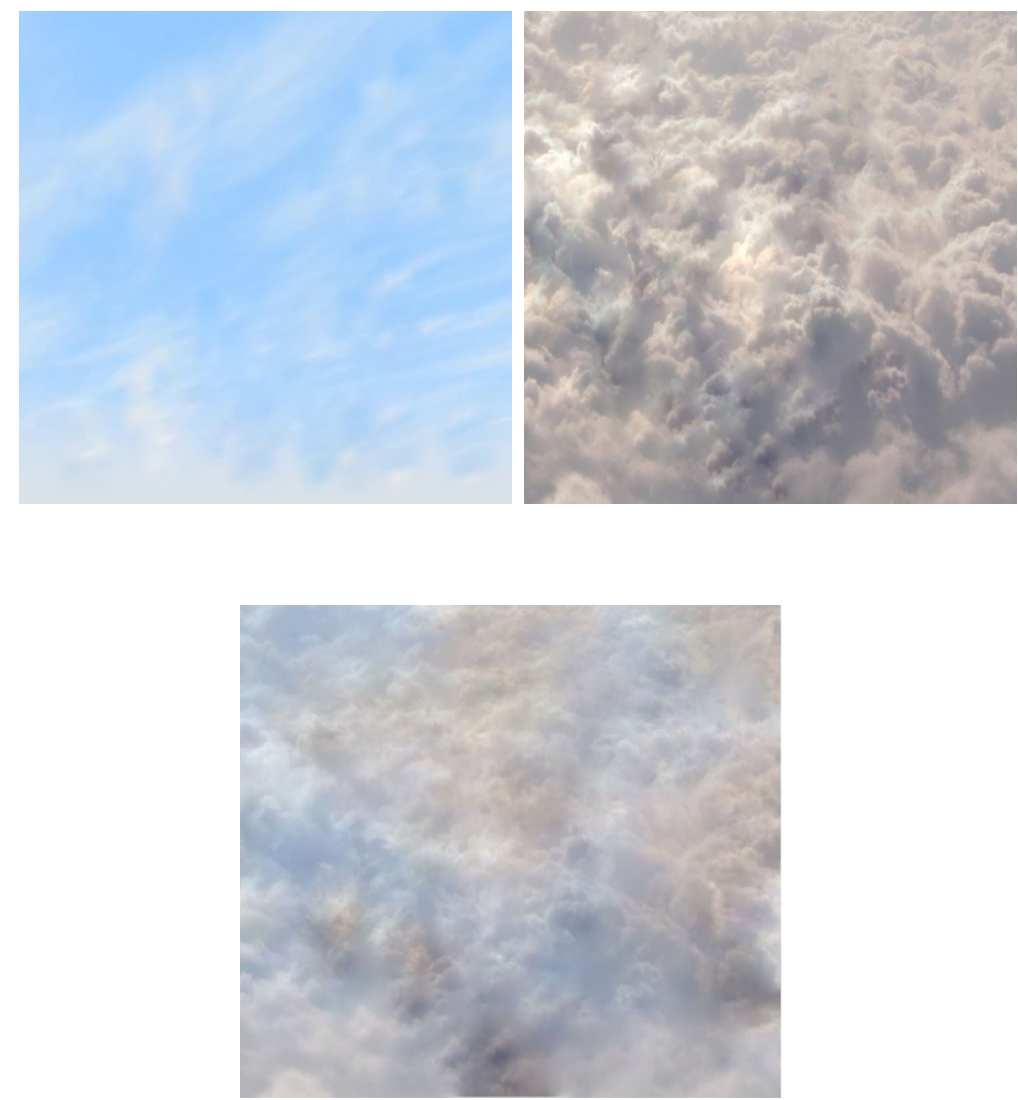

Figure 5.10: Result of blending two textures with different textureness. The images in the upper row are inputs. The bottom image is the output.

For some cases, even after the amplification of the lower coefficient value, it cannot reach the other higher valued coefficient. In such cases the textures with low textureness cannot be preserved in the output.

In an example result in Fig. 5.9, the sand image has much higher textureness than the water image. Throughout the output image the sand texture is mostly preserved. Also in output of Fig. 5.10, the cloud textures are mostly retained.

\subsubsection{Results Showing The Effect Of Sizes Of The SLIC Seg- ments}

The sizes of the SLIC segments affect the result visually. For example in the outputs in the bottom row of Fig. 5.12, the sizes of the SLIC segments are different. The 
sizes of the segments in bottom left output in Fig. 5.12 are smaller than the sizes in bottom right output. If the size of the SLIC segment is large then large chunks of texture contents from inputs are retained. We can observe the yellow chunks from the upper right input in Fig. 5.12, are partially retained in the outputs at the bottom row. In the bottom left output in Fig. 5.12, the yellow chunks came in smaller sizes than in the bottom right output. If the sizes of the segments are small then texture contents from both inputs come in small segments throughout the output texture. Same situation is observed in Fig. 5.11. After several iterations with different sizes of the SLIC segments we got the four different looking outputs in Fig. 5.11. 

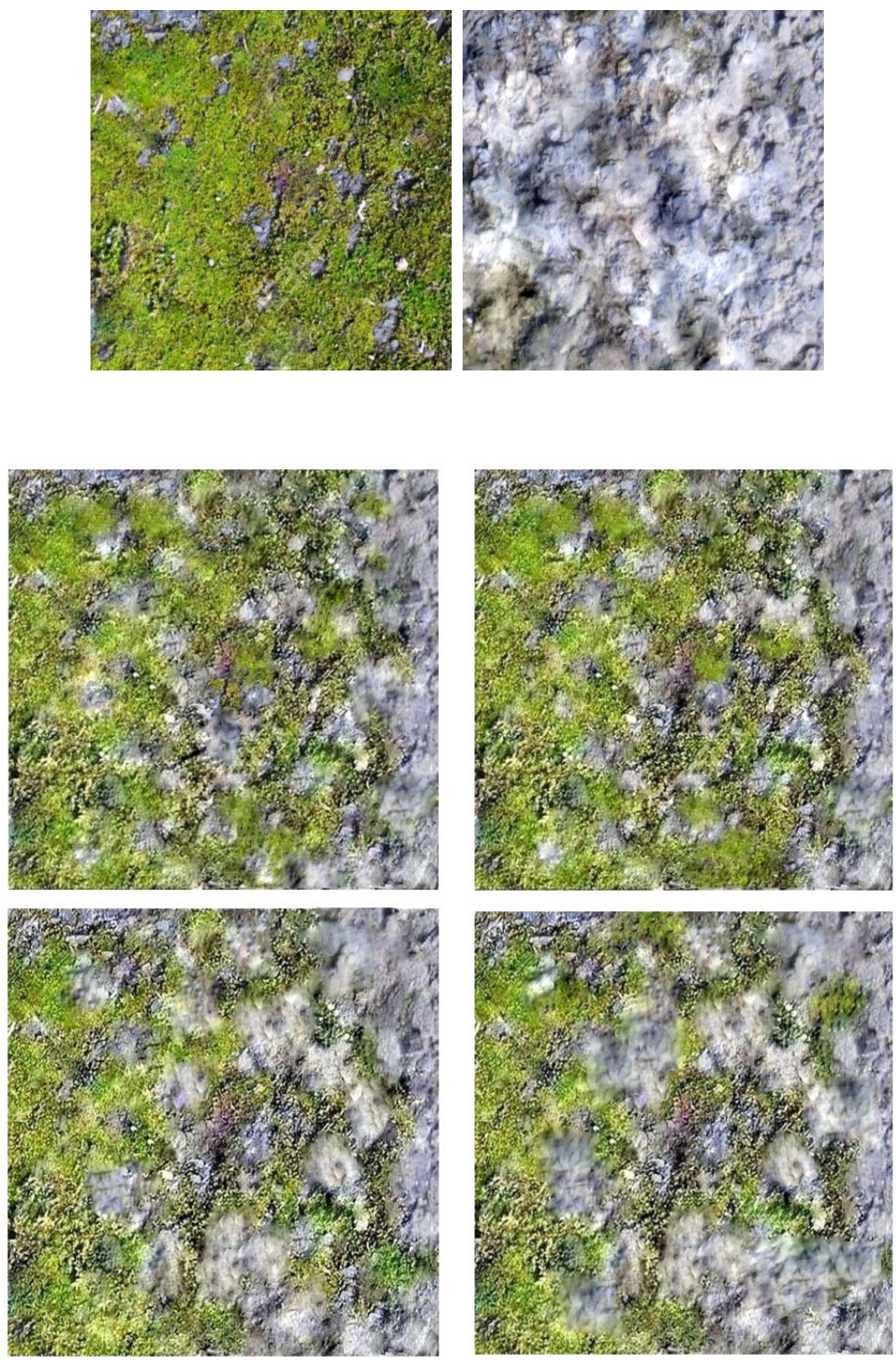

Figure 5.11: Results of our algorithm showing the effect of the different sizes of the SLIC segments. There is change of shape and size of the texture patches according to the size of the SLIC segments. The images in the uppermost row are inputs. The images in the second and third rows are the outputs. 

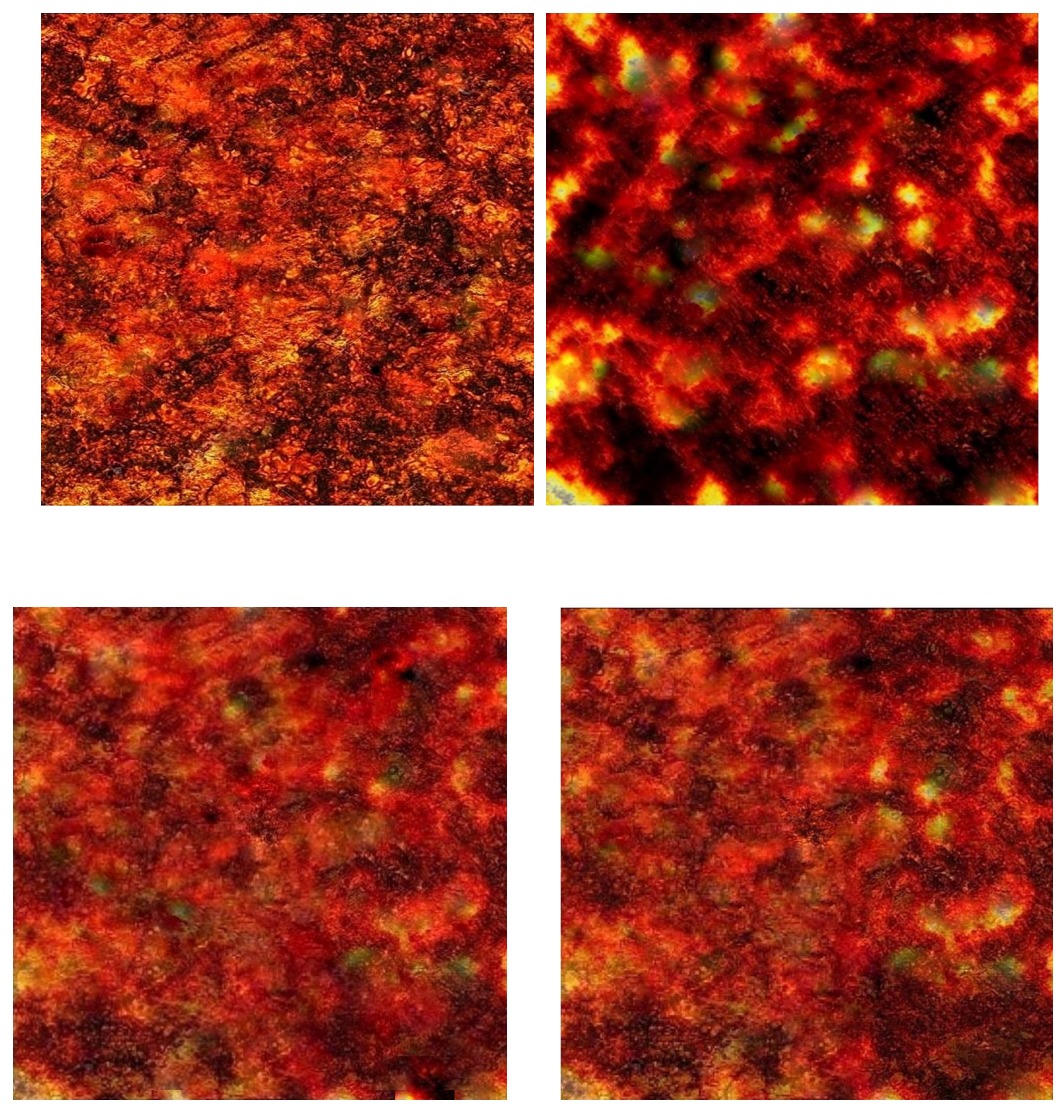

Figure 5.12: Output showing effect of different sizes of SLIC segments. The images in the upper row are inputs. The images in the second and rows are the outputs. Sizes of the SLIC segments are comparatively small in the left output compared to the sizes in the right output. 


\subsubsection{Results With Two Dissimilar Inputs in Terms of Colors and Texture Contents}
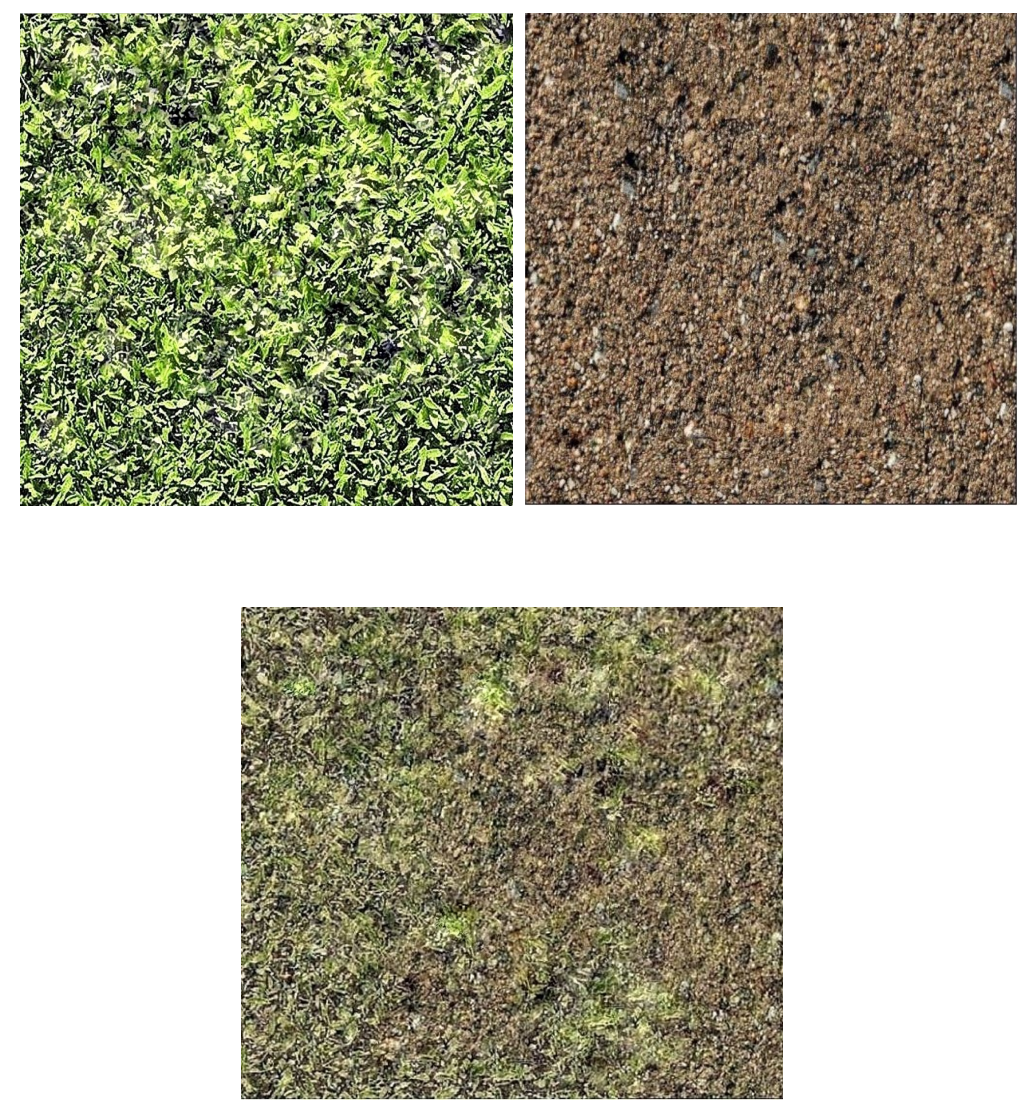

Figure 5.13: Result of our algorithm at the bottom row. Inputs at the first row are generated after the histogram preserving blending operation 

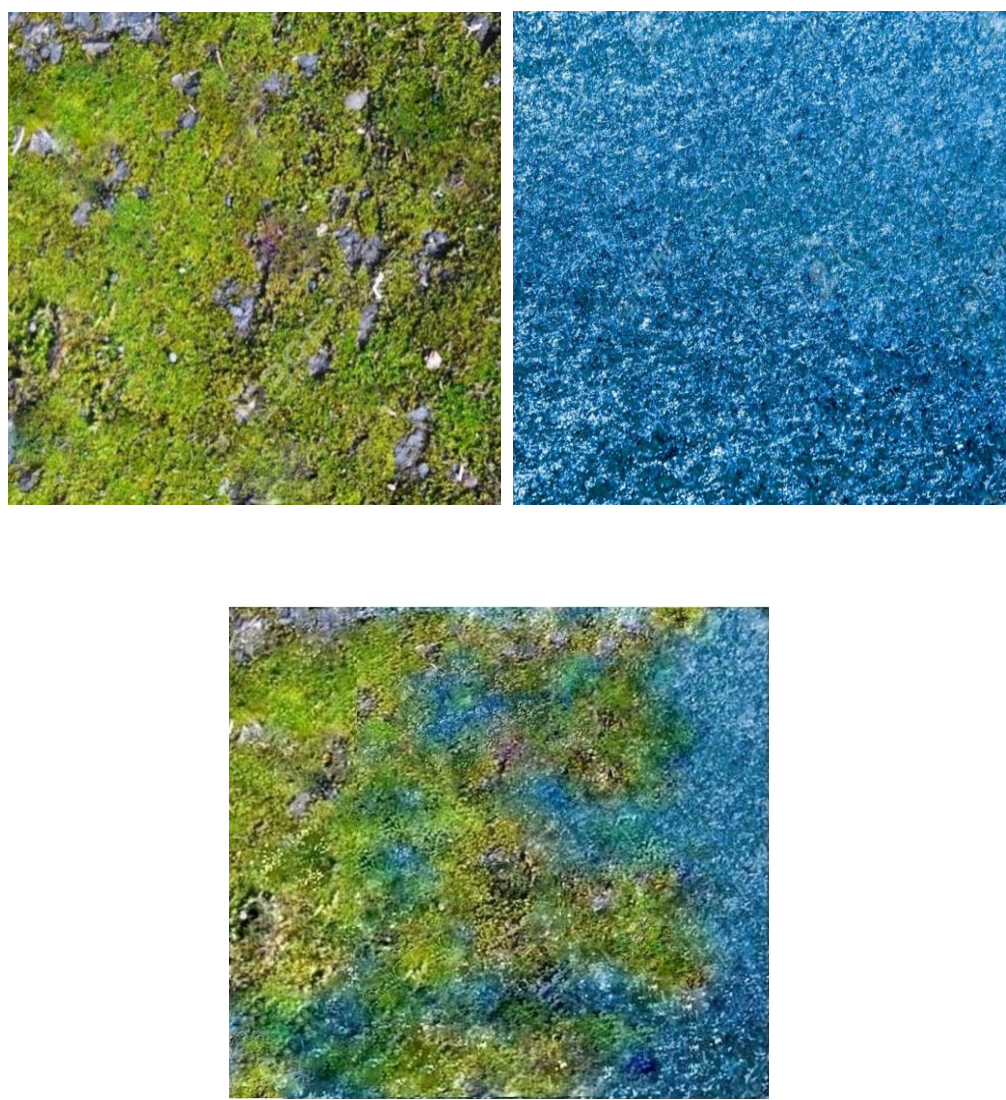

Figure 5.14: Result of our algorithm at the bottom row. Inputs at the first row are generated after the histogram preserving blending operation

In Fig. 5.13 and 5.14, we have taken two inputs with totally different texture and color contents. Merging of pebbles and grass texture in Fig. 5.13 is meaningful in real life even though the grass and the pebbles texture are completely different in color and contents. The random distribution of texture contents from both inputs gives a realistic and meaningful view of the blended texture.

\subsubsection{Results With Two Similar Inputs In Terms of Colors and Texture Contents}



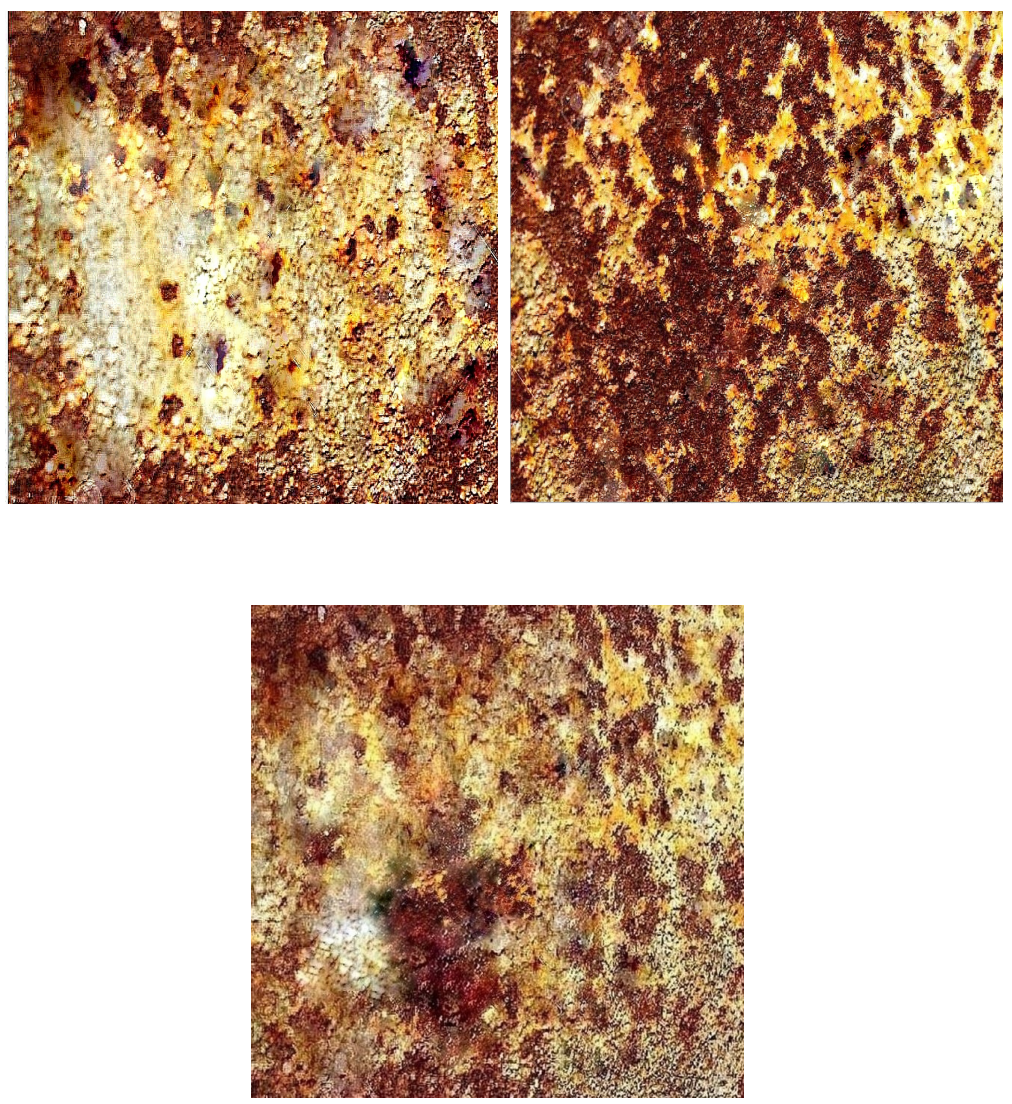

Figure 5.15: Result at the bottom row showing the blend of two similar inputs at the upper row. The inputs are similar in terms of color and texture contents. 

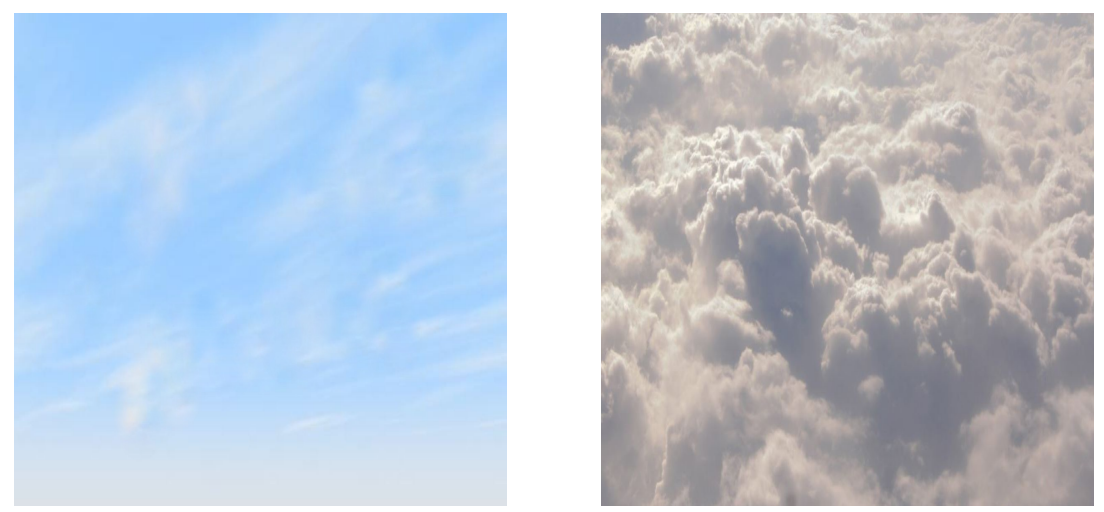

Figure 5.16: Original Inputs
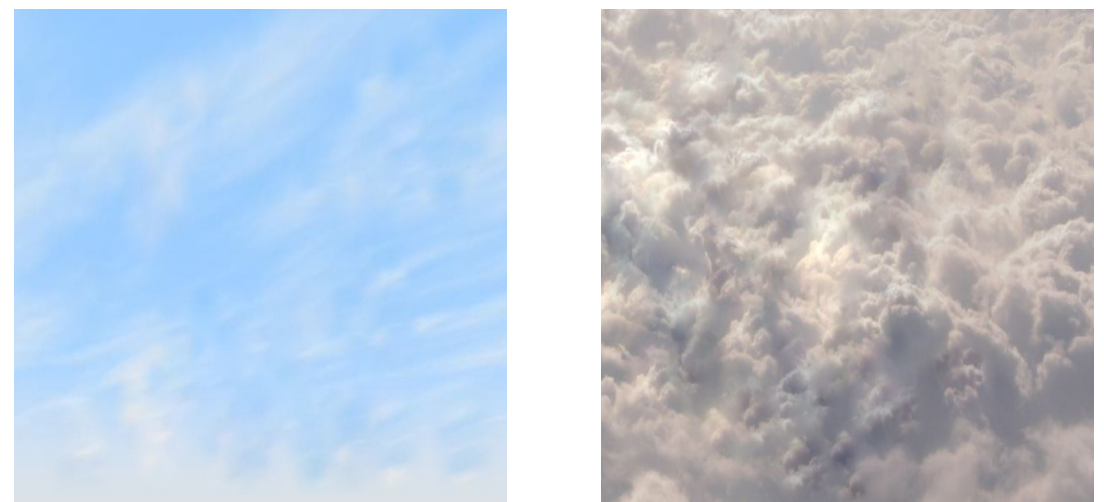

Figure 5.17: Results of the histogram-preserving blending which we used as inputs for our algorithm. 

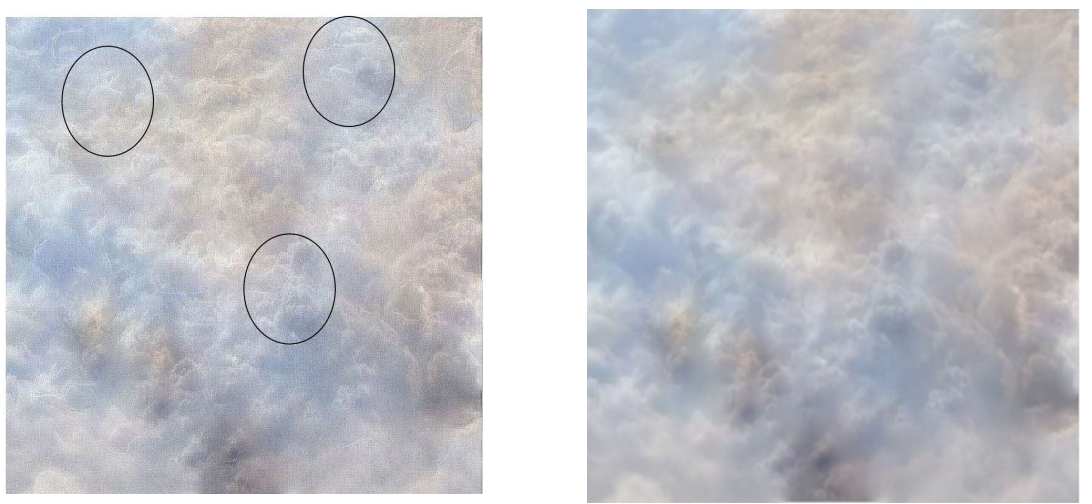

Figure 5.18: Sharp edges observed before smooth blending at halo region (the left image). Result after smooth merging along the SLIC boundaries (the right image)

Result of Fig. 5.12 is the merging of two inputs with similar texture and color contents. Result in Fig. 5.15 is another example of two inputs with similar texture contents. Due to this similarity the output becomes very natural and merging looks continuous and smooth.

After combining all the levels of the resultant Laplacian pyramid we get a greyscale output image. We assign colors to the greyscale output using the lookup table that was generated in the re-implementation process of the histogram preserving blending method. The lookup table stores the RGB pixel values of the input textures. By assigning colors through mapping with the look-up table, we can retain the actual colors of the inputs. But at the regions of the SLIC boundaries, the alpha blending changes the actual colors. This change of colors can be noticed only if two different colored textures lie on either side of the SLIC boundaries. An example of this phenomenon is shown in Fig. 5.18.

In the first result Fig. 5.18, we have shown a result with a clear sky and a cloud texture. Fig. 5.17 is the output from the histogram-preserving algorithm and we used the output as the input of our algorithm. The left image in Fig. 5.18 is the result without any smooth merging in the halo region. Without the smooth merging at the SLIC boundaries, we can observe at some places there are sharp edges between two different texture contents. Sharp edges can also be seen between texture contents 
of completely different color. These sharp edges can be noticed along the boundaries of the SLIC regions. These sharp edges were removed in the right image in Fig. 5.18 with smooth transitioning from one color to other along the SLIC boundary pixels.

\subsubsection{Texture Distribution Map}

In our experiment we calculate the weights which affect the results of the smooth maximum function. We calculate the weights based on the given measure of the proportion of textures from both inputs in the outputs. It means, the users can specify how the textures from each input will be distributed in the output. In our experiment, we specified that the textures from each input will be distributed in such a way that there was a horizontal transition of textures from one input to textures of the other input. But the users may select any distribution of textures in the output.

In Fig. 5.19, it is shown that the textures in the output image can be distributed according to the user specified texture distribution map shown at the bottom right image in Fig. 5.19. The parameter $t$ in our blending equation can be the function of user defined texture distribution. We can observe that the texture distribution in the bottom left output in Fig.5.19, followed the given distribution map. The prominent "9" like distribution in the map is visible in the output. The boundaries of the shapes got smoothly merged. 

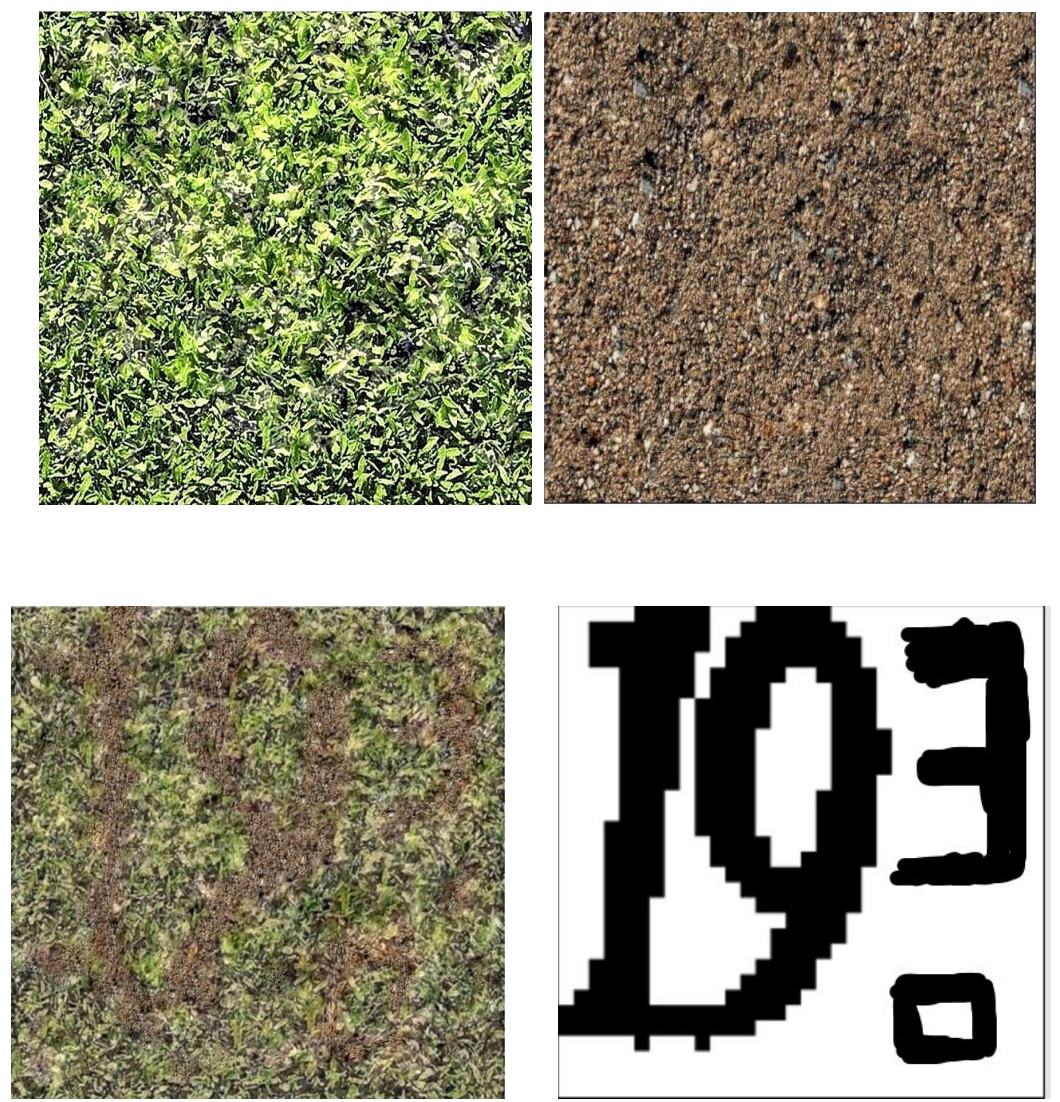

Figure 5.19: Result(at the bottom left) after texture blending where the texture distribution from inputs are based on the user defined texture distribution map (at the bottom right).

\subsection{Discussion}

Every result of our algorithm shows the gradual transition of texture content of one input to the texture content of the other input. There is no sudden transition of texture. Transition of texture happens horizontally from the left edge to the right edge of the output image. Along with the gradual transition, the texture elements are also scattered randomly at some places in every resultant image. The irregular shapes of the SLIC segments also help in the irregular distribution of texture content from both inputs. This contributes in giving a realistic appearance in the texture transition.

We can notice some distortion of texture contents in all of the outputs. This is because, at a certain level of the resultant pyramid $L^{R}$, the blended coefficient might 
be close to the value of coefficient $u$ and at another level, it might be close to value of coefficient $v$. Therefore at a single coefficient location, the blended coefficients might be close to the different coefficients at different levels. When the levels of resultant Laplacian pyramid are combined then in the output greyscale image, the texture content at that particular location get changed completely. If the weighted values of coefficients at a particular location remain higher at all the levels of $L^{R}$, then the actual texture content at that location can be preserved.

This distortion of texture contents is not visually obvious for random phase inputs. This is because of the randomness and lack of any definite structures of the input textures. This distortion is distinctly visible for the non-random phase inputs having regular shaped structures. Therefore, this approach of blending on regular textures is risky.

For random phase inputs having similar color and texture contents, the blending appears smooth and realistic. Sometimes, the blending of two inputs having very much different color and texture contents, gives meaningful outputs. Result showing blending of grass and pebbles in Fig. 5.13, grass and stony ground in Fig. 5.11, sky and cloud in Fig. 5.10, pebbles and water in Fig. 5.8 are example of some meaningful outputs. On the other hand the blending of sand and water texture in Fig. 5.9 has not produced a good blend due to the difference in color and texture contents.

\subsection{Timing Of Different Steps of Algorithm}

In the Table 5.1 below, we show the runtime value of some steps of our algorithm. We aimed at generating the results which serve our goal. That is why some steps are expensive in the algorithm and optimization of those steps are less considered. 
Table 5.1: Timing of algorithm

\begin{tabular}{|c|c|c|}
\hline Steps & Resolution $600 \times 600$ & Resolution $800 \times 800$ \\
\hline $\begin{array}{l}\text { 1. Generating SLIC } \\
\text { segments }\end{array}$ & $0.91 \mathrm{~s}$ & $1.36 \mathrm{~s}$ \\
\hline $\begin{array}{l}\text { 2. Generating lapla- } \\
\text { cian pyramid for each } \\
\text { pair of segments }\end{array}$ & $8.40 \mathrm{~s}$ & $1.43 \mathrm{~s}$ \\
\hline $\begin{array}{l}\text { 3. At each Laplacian } \\
\text { level, smooth maxi- } \\
\text { mum function, ran- } \\
\text { dom location selec- } \\
\text { tion and reconstruc- } \\
\text { tion from the levels }\end{array}$ & $12.80 \mathrm{~s}$ & $17.12 \mathrm{~s}$ \\
\hline $\begin{array}{l}\text { 4. Coloring the } \\
\text { greyscale result with } \\
\text { precomputed lookup } \\
\text { tables and smooth } \\
\text { merging of colors at } \\
\text { halos }\end{array}$ & $9.20 \mathrm{~s}$ & $13.03 \mathrm{~s}$ \\
\hline
\end{tabular}

Table 5.1 shows the timing for each steps of our algorithm with different resolution of inputs.The program was run in a Intel(R) Core(TM)i7-8550U CPU using a single-threaded unoptimized implementation.

\subsection{Failure Cases}

Our algorithm is applicable for some specific category of inputs. For some random phase textures which are very much different in color and texture contents, the result of our algorithm does not provide a meaningful output. The output texture looks very mismatched in the region where the textures are blended. Fig. 5.20 is such an example where the blend of that particular sand texture and water texture is not smooth and natural.

During the coefficient blending, the coefficients get changed. Therefore, there 
is distortion of texture contents. These distortions become more distinct for non-random phase inputs having regular shapes textures. If there are regular, strong, repetitive patterns in the input then this algorithm cannot preserve those definite patterns. In Fig. 5.21 we can notice the regular shapes of bricks got distorted.

We assign colors to the output image by mapping with the lookup table which stores the pixel values of the original input. That is why the colors of the input are preserved. But when two texture with totally different colors are blended, then we do the alpha blend around the halo regions in our algorithm. Due to this alpha blending of colors there are some ghosting or softened discontinuities in the output. The ghosting becomes visually obvious when there is a blending of two different color contents. In Fig.5.20, we can notice such ghosting or softened discontinuities at some places where sand and water texture contents are lying side by side.
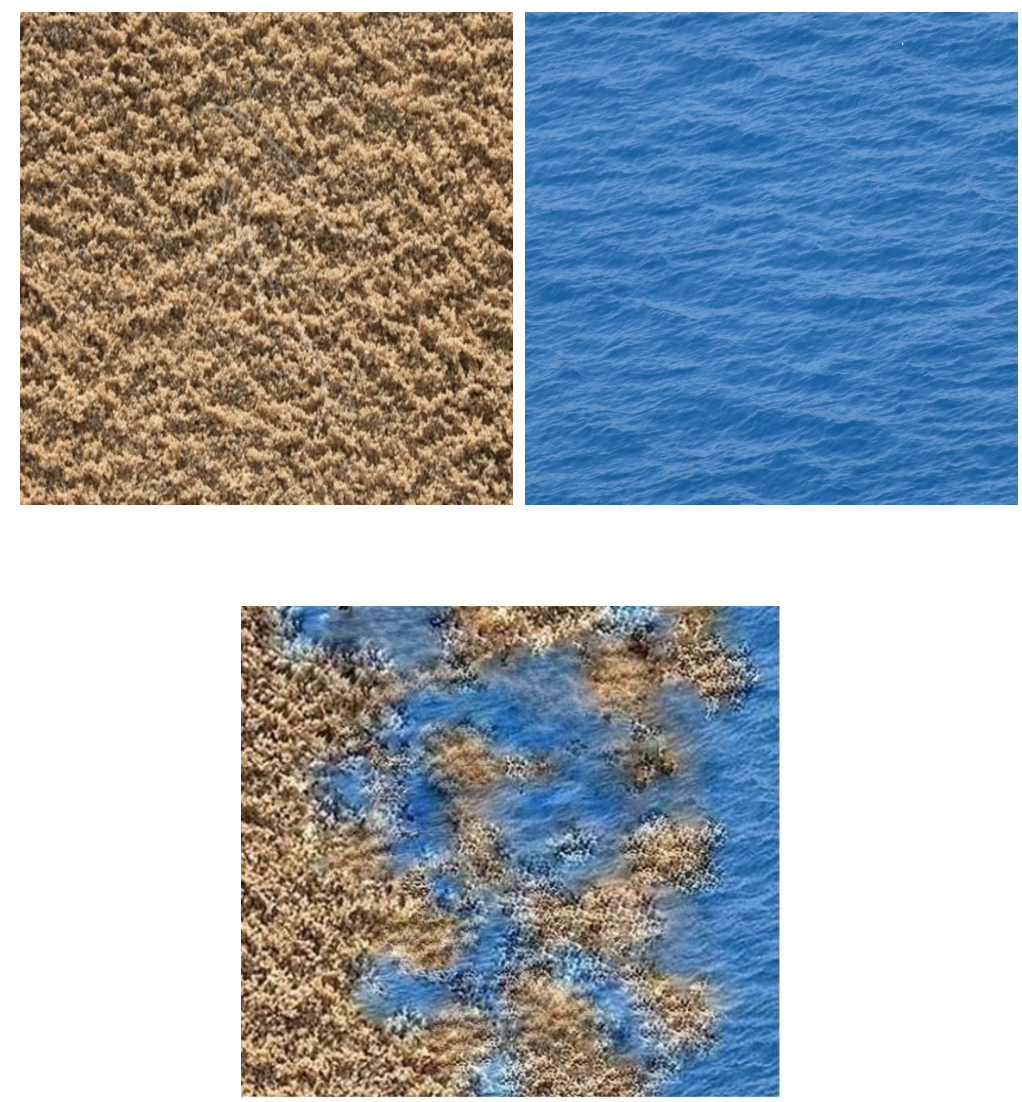

Figure 5.20: Result of blending two textures with different textureness 

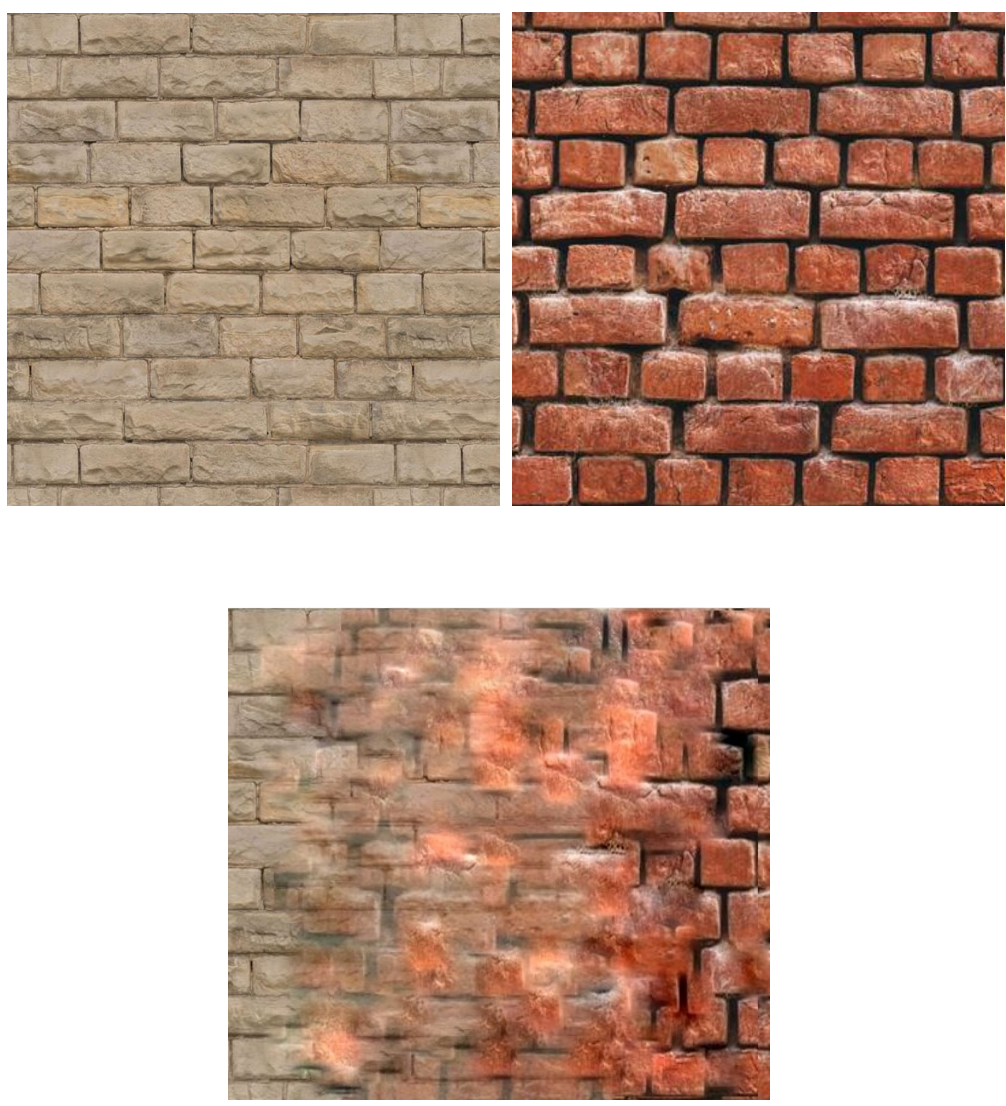

Figure 5.21: Result of blending two textures with regular structure 


\section{Chapter 6}

\section{Conclusion}

\subsection{Summary}

We proposed a texture synthesis approach by merging two images with stochastic texture having random phase and some images with non-random phase textures. The algorithm is applicable for stochastic textures having no well defined, regular structures. In the outputs generated by this algorithm, there is gradual transition from one input textures to textures from the other input. This transition is based on the given measure of how much of the textures from both inputs will be preserved in the output. In our experiments we set this parameter as the spatial location of the pixels in the output image plane. Thus we got the output image where textures towards the left region are more from one input and textures towards the right region are more from the other input. Throughout the middle area there is gradual transition of textures.

Using the SLIC segments we were able to bring the texture contents from both inputs in the form of chunks. That is why, the texture contents from each input were distinguishable even after blending. With the help of the weighted smooth maximum function we are able to put weights on the coefficients of both inputs and generate output coefficients based on the assigned weights. The optimal transport lookup table helped to preserve the actual color of the texture contents.

Our process is applicable only for textures with no regular, well-defined structures. This is the main limitation of our process. It is also unable to preserve long continuous structural contents in the inputs. Despite the limitations, we were able to produce 
some meaningful results. Those results have continuous or gradual progression of textures and the blended region looks realistic and natural.

\subsection{Future Work}

We believe there are many possible and promising directions for the future work. In the results of our algorithm we cannot preserve the texture contents without any distortion. Therefore, an area of further exploration is how to preserve the texture contents from the input without any distortion.

We did alpha blending of colors along the SLIC boundaries in the output for smooth color merging. But this linear blending sometimes creates reduced contrast and ghosting. We look forward to determine a better solution to smoothly merge totally different colors without creating any visual artifacts. We can seek to address some of the limitations of the proposed method. In particular, our strategy for restoring color to the textures was not entirely successful when the input palettes are very dissimilar. More work is needed on this front.

We have merged textures linearly considering the spatial location of each pixel in the output image space. In the future, we can consider using this approach depending on any other scale and also considering other factors for example local contrast, isotropy, correlation, time etc.

In this work, we treated all levels of the Laplacian pyramid the same way. However, it might make sense to investigate different treatments for different levels. Depending on the texture, one level or another may contain more of the structural content, and accounting for this in the merging could produce still better blends. The example of Doyle and Mould [12] is instructive, albeit not in a texture blending context.

Another possibility for future work is to increase the scope of texture types to which the method can be applied. Right now it is most effective for stochastic textures lacking any regular pattern. We would like to explore methods for blending textures 
that have well-defined structure and patterns. 


\section{List of References}

[1] R. Achanta, A. Shaji, K. Smith, A. Lucchi, P. Fua, and S. Süsstrunk, "SLIC superpixels compared to state-of-the-art superpixel methods," IEEE Transactions on Pattern Analysis and Machine Intelligence, vol. 34, no. 11, pp. 2274-2282, 2012.

[2] A. Akl, C. Yaacoub, M. Donias, J.-P. Da Costa, and C. Germain, "A survey of exemplar-based texture synthesis methods," Computer Vision and Image Understanding, vol. 172, 042018.

[3] M. Ashikhmin, "Synthesizing natural textures," in Proceedings of the 2001 Symposium on Interactive 3D Graphics, ser. I3D '01. New York, NY, USA: Association for Computing Machinery, 2001, p. 217-226. [Online]. Available: https://doi.org/10.1145/364338.364405

[4] S. Bae, S. Paris, and F. Durand, "Two-scale tone management for photographic look," in ACM SIGGRAPH 2006 Papers, ser. SIGGRAPH '06. New York, NY, USA: Association for Computing Machinery, 2006, p. 637-645. [Online]. Available: https://doi.org/10.1145/1179352.1141935

[5] N. Bonneel, G. Peyré, and M. Cuturi, "Wasserstein barycentric coordinates: Histogram regression using optimal transport," ACM Trans. Graph., vol. 35, no. 4, Jul. 2016. [Online]. Available: https://doi.org/10.1145/2897824.2925918

[6] Brown Leaves Image, Licensed under CC0 1.0 Universal (CC0 1.0) Public Domain Dedication, Online, accessed 28-04-2020, https://freestocktextures.com/ texture/ground-fall-leaves, 800.html.

[7] P. Burt and E. Adelson, "The Laplacian pyramid as a compact image code," IEEE Trans. Communications, vol. 31, pp. 532-540, 1983.

[8] M. F. Cohen, J. Shade, S. Hiller, and O. Deussen, "Wang tiles for image and texture generation," ACM Trans. Graph., vol. 22, no. 3, p. 287-294, Jul. 2003. [Online]. Available: https://doi.org/10.1145/882262.882265

[9] J. D. Cook, "Soft maximum." [Online]. Available: https://www.johndcook. $\mathrm{com} / \mathrm{blog} / 2010 / 01 / 13 /$ soft-maximum/

[10] D. S. Ebert, F. K. Musgrave, D. Peachey, K. Perlin, and S. Worley, Texturing and Modeling: A Procedural Approach. Morgan Kaufmann, 2003. 
[11] A. Desolneux, Stochastic Geometry. Lecture Notes in Mathematics, E. C. CNRS, CMLA, Ed. Springer, Cham, 2015.

[12] L. Doyle and D. Mould, "Augmenting photographs with textures using the laplacian pyramid," The Visual Computer, pp. 1-12, 2018.

[13] A. A. Efros and T. K. Leung, "Texture synthesis by non-parametric sampling," in Proceedings of the Seventh IEEE International Conference on Computer Vision, vol. 2, 1999, pp. 1033-1038 vol.2.

[14] A. A. Efros and W. T. Freeman, "Image quilting for texture synthesis and transfer," Proceedings of SIGGRAPH 2001, pp. 341-346, August 2001.

[15] Flower Texture, Licensed under CCO 1.0 Universal (CC0 1.0), Online, accessed 28-04-2020, https://freestocktextures.com/texture/blue-hexagons-pattern,902. html.

[16] B. Galerne, A. Leclaire, and L. Moisan, "Texton Noise," Computer Graphics Forum, Jan. 2017. [Online]. Available: https://hal.archives-ouvertes. $\mathrm{fr} /$ hal-01299336

[17] B. Galerne, Y. Gousseau, and J.-M. Morel, "Random Phase Textures: Theory and Synthesis," IEEE Transactions on Image Processing, vol. 20, no. 1, pp. 257 - 267, Jan. 2011. [Online]. Available: https://hal.archives-ouvertes.fr/ hal-00418389

[18] L. A. Gatys, A. S. Ecker, and M. Bethge, "Texture synthesis using convolutional neural networks," in Proceedings of the 28th International Conference on Neural Information Processing Systems - Volume 1, ser. NIPS'15. Cambridge, MA, USA: MIT Press, 2015, p. 262-270.

[19] G. Gilet, B. Sauvage, K. Vanhoey, J.-M. Dischler, and D. Ghazanfarpour, "Local random-phase noise for procedural texturing," ACM Trans. Graph., vol. 33, pp. 195:1-195:11, 112014.

[20] Granite Image, GNU General Public License, Online, accessed 28-04-2020, http: //libnoise.sourceforge.net/examples/textures/images/granite/plane.jpg.

[21] D. J. Heeger and J. R. Bergen, "Pyramid-based texture analysis/synthesis," in Proceedings of the 22nd Annual Conference on Computer Graphics and Interactive Techniques, ser. SIGGRAPH '95. New York, NY, USA: Association for Computing Machinery, 1995, p. 229-238. [Online]. Available: https://doi.org/10.1145/218380.218446

[22] E. Heitz and F. Neyret, "High-performance by-example noise using a histogrampreserving blending operator," Proc. ACM Comput. Graph. Interact. Tech., vol. 1, no. 2, Aug. 2018. [Online]. Available: https://doi.org/10.1145/3233304

[23] A. Hertzmann, C. E. Jacobs, N. Oliver, B. Curless, and D. H. Salesin, "Image analogies," in Proceedings of the 28th Annual Conference on Computer Graphics and Interactive Techniques, ser. SIGGRAPH '01. New York, NY, USA: Association for Computing Machinery, 2001, p. 327-340. [Online]. Available: https://doi.org/10.1145/383259.383295 
[24] Ivy Autumn Leaves Textures, Licensed under CC0 1.0 Universal (CC0 1.0), Online, accessed 28-04-2020, https://freestocktextures.com/texture/ ivy-autumn-leaves, 741.html.

[25] J.Salzarulo, Licensed under CCO 1.0 Universal (CCO 1.0), Online, accessed 2804-2020, https://freestocktextures.com/texture/pebbles-on-the-beach,908.html.

[26] B. Julesz, "Visual pattern discrimination," IRE Transactions on Information Theory, vol. 8, no. 2, pp. 84-92, 1962.

[27] B. Julesz, "Textons, the elements of texture perception, and their interactions," Nature, vol. 290, pp. 91-97, 1981.

[28] B. Julesz, E. Gilbert, L. Shepp, and H. Frisch, "Inability of humans to discriminate between visual textures that agree in second-order statisticsrevisited," Perception, vol. 2, no. 4, p. 391-405, 1973. [Online]. Available: https://doi.org/10.1068/p020391

[29] V. Kwatra, I. Essa, A. Bobick, and N. Kwatra, "Texture optimization for example-based synthesis," ACM Trans. Graph., vol. 24, pp. 795-802, 072005.

[30] V. Kwatra, A. Schödl, I. Essa, G. Turk, and A. Bobick, "Graphcut textures: Image and video synthesis using graph cuts," ACM Transactions on Graphics, SIGGRAPH 2003, vol. 22, no. 3, pp. 277-286, July 2003.

[31] J.-P. Lewis, "Texture synthesis for digital painting," SIGGRAPH Comput. Graph., vol. 18, no. 3, p. 245-252, Jan. 1984. [Online]. Available: https://doi.org/10.1145/964965.808605

[32] S. Paris, S. W. Hasinoff, and J. Kautz, "Local laplacian filters: Edge-aware image processing with a laplacian pyramid," Commun. ACM, vol. 58, no. 3, p. 81-91, Feb. 2015. [Online]. Available: https://doi.org/10.1145/2723694

[33] N. Pavie, G. Gilet, J.-M. Dischler, and D. Ghazanfarpour, "Procedural texture synthesis by locally controlled spot noise," 052016.

[34] K. Perlin, "An image synthesizer," SIGGRAPH Comput. Graph., vol. 19, no. 3, p. 287-296, Jul. 1985. [Online]. Available: https://doi.org/10.1145/325165.325247

[35] J. Portilla and E. P. Simoncelli, "A parametric texture model based on joint statistics of complex wavelet coefficients," International Journal of Computer Vision, vol. 40, pp. 49-70, 2000.

[36] M. Ralev, Free moss texture Stock Photo, Online, accessed 28-04-2020, https: //www.freeimages.com/photo/moss-texture-1178742.

[37] Small Leaves Image, Licensed under CC0 1.0 Universal (CC0 1.0) Public Domain Dedication, Online, accessed 28-04-2020, https://freestocktextures.com/texture/ green-nature-groundcover, 613.html. 
[38] G. Turk, "Generating textures on arbitrary surfaces using reaction-diffusion," in Proceedings of the 18th Annual Conference on Computer Graphics and Interactive Techniques, ser. SIGGRAPH '91. New York, NY, USA: Association for Computing Machinery, 1991, p. 289-298. [Online]. Available: https://doi.org/10.1145/122718.122749

[39] L. Wang and D.-C. He, "Texture classification using texture spectrum," Pattern Recognition, vol. 23, no. 8, pp. 905 - 910, 1990. [Online]. Available: http://www.sciencedirect.com/science/article/pii/0031320390901358

[40] L.-Y. Wei, "Texture synthesis by fixed neighborhood searching," Ph.D. dissertation, Stanford, CA, USA, 2002, aAI3038169.

[41] S. Zeller, Licensed under CCO 1.0 Universal (CC0 1.0), Online, accessed 28-042020, https://freestocktextures.com/texture/blue-hexagons-pattern,902.html.

[42] J. Zhang, K. Zhou, L. Velho, B. Guo, and H.-Y. Shum, "Synthesis of progressively-variant textures on arbitrary surfaces," ACM Trans. Graph., vol. 22, no. 3, p. 295-302, Jul. 2003. [Online]. Available: https: //doi.org/10.1145/882262.882266

[43] Y. Zhou, H. Shi, D. Lischinski, M. Gong, J. Kopf, and H. Huang, "Analysis and controlled synthesis of inhomogeneous textures," Computer Graphics Forum (Proceedings of Eurographics), vol. 36, no. 2, pp. 199-212, 2017.

[44] Y. Zhou, Z. Zhu, X. Bai, D. Lischinski, D. Cohen-Or, and H. Huang, "Non-stationary texture synthesis by adversarial expansion," ACM Trans. Graph., vol. 37, no. 4, Jul. 2018. [Online]. Available: https://doi.org/10.1145/ 3197517.3201285 